\title{
Morphological approaches in studying fungi: collection, examination, isolation, sporulation and preservation
}

\author{
Senanayake $\mathrm{IC}^{1,2,3,4}$, Rathnayaka $\mathrm{AR}^{3,4,13}$, Marasinghe $\mathrm{DS}^{3,4}$, Calabon $\mathrm{MS}^{3,4}$, \\ Gentekaki $\mathbf{E}^{3,4}$, Lee $\mathbf{H B}^{5}$, Hurdeal $\mathbf{V G}^{3,4}$, Pem $\mathrm{D}^{3,4}$, Dissanayake $\mathbf{L S}^{3,4,6}$, \\ Wijesinghe $\mathrm{SN}^{3,4}$, Bundhun $\mathrm{D}^{3,12,14}$, Nguyen $\mathrm{TT}^{5}$, Goonasekara ID $^{3,4}$, \\ Abeywickrama $\mathrm{PD}^{3,4,7}$, Bhunjun $\mathrm{CS}^{3,4}$, Jayawardena $\mathbf{R S}^{3,4}$, Wanasinghe $\mathrm{DN}^{3,4,9}$, \\ Jeewon $\mathbf{R}^{8}$, Bhat $\mathrm{DJ}^{10,11}$ and Xiang $\mathrm{MM}^{1}$
}

\footnotetext{
${ }^{1}$ Innovative Institute of Plant Health, Zhongkai University of Agriculture and Engineering, Haizhu District, Guangzhou 510225, China

${ }^{2}$ Guangdong Provincial Key Laboratory for Plant Epigenetics, College of Life Science and Oceanography, Shenzhen University, 3688, Nanhai Avenue, Nanshan, Shenzhen, Guangdong 518055, China

${ }^{3}$ Center of Excellence in Fungal Research, Mae Fah Luang University, Chiang Rai 57100, Thailand

${ }^{4}$ School of Science, Mae Fah Luang University, Chiang Rai 57100, Thailand

${ }^{5}$ Department of Agricultural Biological Chemistry, College of Agriculture and Life Sciences, Chonnam National University, Gwangju 61186, Korea

${ }^{6}$ Engineering Research Center of the Utilization for Characteristic Bio-Pharmaceutical Resources in Southwest, Ministry of Education, Guizhou University, Guiyang, Guizhou 550025, China

${ }^{7}$ Beijing Key Laboratory of Environment Friendly Management on Fruit Diseases and Pests in North China, Institute of Plant and Environment Protection, Beijing Academy of Agriculture and Forestry Sciences, Beijing, 100097, China

${ }^{8}$ Department of Health Sciences, Faculty of Science, University of Mauritius, Reduit, Mauritius

${ }^{9}$ CAS Key Laboratory for Plant Biodiversity and Biogeography of East Asia (KLPB), Kunming Institute of Botany, Chinese Academy of Science, Kunming, Yunnan, 650201, China

${ }^{10}$ Formerly, Department of Botany, Goa University, Goa, India

${ }^{11}$ No. 128/1-J, Azad Co-Op Housing Society, Curca, Goa Velha, India

${ }^{12}$ Department of Plant Pathology, Agriculture College, Guizhou University, Guiyang, Guizhou 550025, China

${ }^{13}$ Department of Plant Medicine, National Chiayi University, 300 Syuefu Road, Chiayi 60004, Taiwan

${ }^{14}$ Division of Plant Pathology, Department of Entomology and Plant Pathology, Faculty of Agriculture, Chiang Mai University, Chiang Mai, 50200 Thailand
}

Senanayake IC, Rathnayaka AR, Marasinghe DS, Calabon MS, Gentekaki E, Lee HB, Hurdeal VG, Pem D, Dissanayake LS, Wijesinghe SN, Bundhun D, Nguyen TT, Goonasekara ID, Abeywickrama PD, Bhunjun CS, Jayawardena RS, Wanasinghe DN, Jeewon R, Bhat DJ, Xiang MM 2020 - Morphological approaches in studying fungi: collection, examination, isolation, sporulation and preservation. Mycosphere 11(1), 2678-2754, Doi 10.5943/mycosphere/11/1/20

\begin{abstract}
Traditionally, fungal taxonomy was based on observable phenotypic characters. Recent advances have driven taxonomic conclusions towards DNA-based approaches and these techniques have corresponding pros and cons. Species concepts must therefore rely on incorporated approaches of genotypic, phenotypic and physiological characters and chemotaxonomy. Examination and interpretation of morphological characters however vary from person to person. Standardized procedures are used in the taxonomic study of fungi and general practices of phenotypic approaches are herein outlined. It is not possible to detail all techniques for all fungi and thus, this paper emphasizes on microfungi. Specimen collection is the initial step in any
\end{abstract}


taxonomic study and all taxonomic information are gathered from the specimens. Therefore, guidelines are provided for the collection, data recording and storage of specimens. Morphological examination, microscopy, photography and descriptions of specimens are important for fungal identification. Hence, techniques for staining, mounting and slide preparation are explained. In addition, obtaining pure cultures from specimens and maintaining those isolates for future studies are challenging. Isolation techniques are numerous and often complicated. Good techniques need to isolate a maximum number of strains from a specimen and obtain the desired taxon, while excluding all others. Methods to isolate microfungi including basal fungi, hyphomycetes, coelomycetes, ascomycetes, plant pathogens, soil fungi, air-borne fungi, epiphytes and endophytes are detailed herein. Sporulating cultures are useful to describe the morphological characters of relevant fungi, but sometimes these characters are absent or difficult to find on natural substrates and it is also difficult to link same fungal organisms based on sexual and asexual morphs. The techniques that induce sporulation of different fungal groups are explained and discussed. Specimens, protologues or descriptions, diagrams, illustrations, cultures and DNA sequences need to be deposited at accessible repositories and guidelines are provided for such deposition. The available data are used in future studies. Furthermore, preservation of cultures and specimens is essential. Cultures are used in DNA extraction, mating or cultivation studies, sporulation and metabolites extraction. Colony characters are often significant from each other and sporulated, dry cultures and specimens represent the type status of the desired fungus. Therefore, culture and specimen preservation techniques of different fungal groups are discussed.

Keywords - Ex situ preservation - Isolates - Morphology - Mycotaxonomy - Specimen collections

\section{Introduction}

The term fungus was directly adopted from the Latin word "fungus" (Simpson 1979). The scientific study of fungi is believed to have originated in 1836 with Miles Joseph Berkeley's publication (Ainsworth 1976). Earlier, taxonomists contemplated that fungi were closely related to plants, based on their similar morphology and growth habitat. Later, it was realized that fungi are a separate kingdom, which diverged around one billion years ago (Baldauf \& Palmer 1993, Bruns et al. 2006, Parfrey et al. 2011). Around 144,000 species of fungi have so far been formally described (Willis et al. 2018, Wijayawardene et al. 2020), but it has been estimated that there may be 2.2 to 3.8 million species (Hawksworth \& Lücking 2017) and therefore, the actual number is far from certain (Hyde et al. 2020a). Traditionally, fungal species have been distinguished by different approaches and concepts based on morphology, physiology, biochemistry or reactions to chemical tests.

Classification based on phenotypic characters is the most common traditional method used in defining fungi and it was improved with innovations in microscopes in the $19^{\text {th }}$ century. However, fungal spores and their germ tubes were observed by Giambattista Della Porta in 1588 for the very first time. A preliminary classification was established for mushrooms by Persoon (1794) and Fries (1873) expanded this using spore colour and microscopic characteristics such as arrangement of the hymenophore, pores, gills and teeth. Numerous archetypal publications have used morphology to classify higher to lower taxonomic levels of fungi, such as Hughes (1953), Kohlmeyer \& Kohlmeyer (1979), von Arx \& Müller (1975), Barr (1978), Carmichael (1962), Sutton (1980) and Barr (1987). The results of morphology-based taxonomic studies are also important and used in other research areas, such as fungal biochemistry, biotechnology, bioremediation, physiology and plant pathology (Ali 1962, Hyde \& Alcorn 1993, Ali-Shtayeh \& Jamous 2000, De Souza \& Declerck 2003, Duong et al. 2008, Evidente et al. 2008, Hyde \& Soytong 2008).

Modern mycotaxonomy has moved forward using morphology with a combination of chemotaxonomy, phylogeny, genetics, ecology and molecular biology (Senanayake et al. 2017b, 2018, Manawasinghe et al. 2019, Hyde et al. 2020a, Phukhamsakda et al. 2020a, Samarakoon et al. 2020, Wibberg et al. 2020). Utilization of sequence data for phylogenetic, biological, genetic and 
evolutionary analyses, has provided insights into the diversity and inter and intra-relationships of fungal groups (Hongsanan et al. 2017, Hyde et al. 2020b). These have challenged, but also complemented traditional morphology-based fungal classification over time (Ekanayaka et al. 2017).

\section{Why phenotypic classification is important?}

Our knowledge of fungal taxonomy is primarily based on morphology-based classification (Hyde et al. 2010). It is initially important to use phenotypic approaches and followed by other methodologies such as molecular, chemical, ecological or physiological analyses (Manawasinghe et al. 2019). However, some technologies are not yet affordable or convenient to use in all the basic laboratories. The cost of novel technologies is relatively high. Morphological analyses are however, low in cost and results are obtained rapidly (Hyde et al. 2010). In cases where, there is a lack of sequence data or a limited amount of a specimen is available, then morphological data may still be useful (Chethana et al. 2020). There are also numerous wrongly named sequences or sequence data with errors in GenBank and morphology helps to resolve the taxonomy of them at this point (Hyde et al. 2010). Therefore, morphology is still the most common and reliable procedure to study fungi.

A large number of extant species do not have molecular data and most taxonomic databases contain morphological and ecological data such as nutrient mode, ecological distribution, hosts, and substrates (Muthukrishnan et al. 2012, Senanayake et al. 2015). Therefore, researchers can compare those data across and among different taxonomic or ecological groups of fungi even in the absence of molecular data (Schmit \& Lodge 2005). These are important considerations for large-scale research. In addition, researchers in developing nations are unable to obtain large amounts of molecular data because of its cost (Mueller et al. 2004a).

Morphology based fungal taxonomy is essential to determine the global mycota (Hibbett et al. 2011, Gupta et al. 2013). In the last two decades, a huge amount of discovered new species were introduced based on both phenotypic and genotypic characters (Gupta et al. 2013). DNA based systematics has confirmed much of the phenotypic taxonomy and under most circumstances they have been found to be complementary. Protologues of novel taxa now require English (or Latin) diagnosis for validation (International Code of Nomenclature for algae, fungi and plants (Shenzhen Code), Turland et al. 2018). Therefore, phenotypic taxonomy is basic, applicable in many cases, rapid and inexpensive.

\section{Negative aspects of conventional morphology-based taxonomy}

Morphology-based taxonomy sometimes may not resolve species accurately due to overlapping characters, a high degree of phenotypic plasticity, cryptic species, and occurrence of different morphs for the same taxa (Hyde et al. 2016, Jeewon \& Hyde 2016, Wijayawardene et al. 2016). Cryptic species simply refer to the fungal species that have similar morphological characters, but are genetically different (Williams et al. 1990, Roeckel-Drevet et al. 1997, Wijayawardene et al. 2014) while phenotypic stasis forms the genetic variations by natural selection, mutation and recombination (Chethana et al. 2020). Morphology of a single species sometimes shows slight variations under different environmental conditions, geographical regions, hosts and different life modes (Vasilyeva \& Stephenson 2010, Lücking 2019). Pleomorphism is another aspect, which is the ability of some fungi to alter their morphology, biological functions or reproductive modes in response to environmental conditions (Wingfield et al. 2012, Peršoh 2015). Homoplasy may also occur in some fungal species which are shared morphological characters by a set of species, but not present in their common ancestor (Chethana et al. 2020). Therefore, it is not always possible to separate taxa based on morphological comparisons (Maharachchikumbura et al. 2014, Udayanga et al. 2014).

Sampling errors and personal experience of taxonomists are also affected on morphological characterization (Bortolus 2008). However, these errors can be eliminated by molecular analyses. Some taxa may not grow or produce reproductive structures on artificial media and rarely produce sexual or asexual structures in natural settings. Therefore, these taxa may be overlooked in 
traditional morphological characterization even though they could be important members of the fungal community (Hyde \& Jones 2002). Morphological data is however, inappropriate for largescale environmental surveys. Classical sampling is more time consuming than molecular techniques (Straatsma et al. 2001). Considerable taxonomic expertise is required for classical sampling because species are identified based on morphology (Phukhamsakda et al. 2020b). In morphologybased methodology, sexual and asexual morphs are identified as two distinct species, while this is not an issue with molecular data (Taylor 2011, Wingfield et al. 2012).

\section{What is the need of this paper?}

The protocols for morphological analyses of fungi are scattered in various papers and different researchers follow different methods. Therefore, it is difficult to compare the results because the followed methodologies are different. Hence, gathering of all the protocols and presenting the standardized protocols are required. Each group of fungi has its own protocols and it is impossible to include all of these in a single paper, and therefore, this paper reviews the methods only for microfungi. Readers should refer to the various manuals available for specific groups of fungi. Examples include yeasts (Arai et al. 1977, Liu et al. 2011, Suzuki et al. 2018), aeroaquatic fungi (Fisher \& Webster 1981, Webster \& Descals 1981, Hawksworth 1991), mushrooms (Mueller et al. 2004b, Leonard 2010, Taylor \& Ellison 2010), medical mycology (Beneke \& Rogers 1980, Homei 2006, Pihet et al. 2009, Köhler et al. 2015, Sullivan \& Moran 2015, Yu et al. 2017), Discomycetes (Barr 1990, Ekanayaka et al. 2017), lichens (Sanders 1997, Gargas et al. 1995, Muggia \& Grube 2018), smuts and rusts (McAlpine 1906, Vánky \& Shivas 2008, Shivas et al. 2014) and plant parasitic fungi (Wennström 1993, Wingfield et al. 2012).

\section{Objectives and outcomes}

The protocols of morphological examination, isolation, sporulation and ex-situ preservation of microfungi including saprobic sexual and asexual morphs of ascomycetes, endophytic, epiphytic, airborne, soil, phytopathogenic and basal fungi are reviewed. Sampling techniques, getting permits and access to collecting sites, field tools, temporary storage, transport and preparation of specimens for examination are discussed in detail under specimen collection and examination. In morphological examination and characterization, several subtopics are discussed as sectioning of specimens, mounting, staining, microscope slide preparation, photography and illustrating. As well as, isolation and sporulation techniques for microfungi are also discussed. In addition, cultures and specimen preservation, rules and regulations for deposition and transportation are discussed. Limitations and shortcomings of conventional methods are discussed and guidelines and solutions are provided.

\section{Fungal specimen collection and examination}

Fungi are ubiquitous in terrestrial and aquatic habitats including extreme environments (Selbmann et al. 2005, Maharachchikumbura et al. 2016). They have various modes of nutrition, importantly as saprobes, phytopathogens, animal and human pathogens, soil or dung inhabitants, endophytes, epiphytes, symbionts, lichenicolous and fungicolous (Lawrey \& Diederich 2003, Chomnunti et al. 2014, Kim et al. 2017, Sun et al. 2019, Hyde et al. 2020b). Therefore, it is necessary to be acquainted with modes of life and life cycles before collecting fungal specimens. Samples are generally collected based on the objectives of the research, such as ecology-, niche-, host-, geological distribution- and taxon-based studies (Guarro et al. 1999, Zhang et al. 2015, Girometta et al. 2020).

Sample collection is a skill, which improves with experience. A beginner who learns to collect specimens may not generally collect like an expert. The trainee mycologists always collect "macro" members of Diatrypaceae, Xylariaceae and Hysteriaceae species as these are conspicuous (Hyde et al. 2010, 2020a, Leonard 2010, Hernández-Restrepo et al. 2017). With time, the beginner will improve their skill to locate inconspicuous microfungi. It is important to establish which taxa to look for by reading about their general morphology. Information on habitat and modes of life 
will help the collectors to find the desired taxa (Leonard 2010). Climate, seasons and weather conditions are important. Seasonality affects fungal composition, species richness and species diversity (Tokumasu 1998, Maria \& Sridhar 2004, Muthukrishnan et al. 2012). For example, some saprobic fungi may occur more often and at a higher frequency during the rainy season (Zhou \& Hyde 2002), while they may produce sexual morphs with thick-walled spores when the environment is dry. Fungal diversity differs from terrestrial to aquatic habitats even when those habitats border each other (Cai et al. 2006). Though, there are a small number of dominant species common to both habitats, a considerable amount of species is unique in each ecosystem (Jobard et al. 2010).

Fungal specimen collection is important in many ways. Fresh fungal specimens are the basic biological material for mycologists and taxonomists to study (Prance \& Fechner 2017). All specimens of taxonomically novel taxa are conserved as vouchers for scientific research including taxonomic, ecological, biochemical analyses and reference specimens for accurate identification of fungi (Raja et al. 2017, Wu et al. 2019). Type specimens are the representative material of the taxa and it is possible to designate epitypes, paratypes and syntypes using dried collections (Ariyawansa et al. 2014). Host, locality, time and distribution of taxa are documented with specimens and this information merges with most ecological studies.

\section{Permits and access}

Collecting specimens in national parks, state forests and protected areas is not allowed in many countries (Leonard 2010). Sometimes, it is possible to obtain a permit specifying the collecting sites, duration and purpose of collecting for scientific study, which may help in gaining access. Permission is requested from the owner or the authority to access and traverse the territory before entering private and farm lands (Dudley 2008, Dudley et al. 2013).

\section{Field tools}

Collectors should wear enough protective tools for personal care, such as sunscreen cream, hats, long-sleeved shirts and trousers, insect repellent, sturdy shoes and should bring a first-aid kit, water and foods. There is usually no risk in handling fungi except some poisonous mushroom species and human-pathogenic microfungi (De Mattos-Shipley et al. 2016, Hyde et al. 2019). However, some people may have dermatological or respiratory allergies to fungal spores and exudates.

The following tools (Fig. 1) should be taken to the field for collecting, recording and storage of samples.

\section{Pocket knives, forceps, soft paint brushes or trowels}

If the species inhabits twigs, leaves, flowers, seeds or soil, then they are collected carefully and placed in a container. If the species is associated with bark of woody trees, then bark tissues should be removed from the tree carefully using a knife (Mueller et al. 2004c). It is important to remove entire specimens from their substrate without damaging the specimen.

2. Suitable containers, Aluminum foil, water-proof paper bags or zip-lock polythene bags

Plastic boxes are better for hyphomycetes, large specimens or specimens with visible fruiting bodies. Large microfungi such as Xylariales specimens and some basal fungi are mostly stored in handyman's boxes with multiple compartments or fisherman's boxes. Cotton, Tyvek, or paper bags can be used for small specimens and zip-lock bags are used for soil samples (Wardle \& Lavelle 1997). Generally, plastic bags are not recommended to transport or store soft, moisten fungal specimens, because the fungi deteriorate rapidly due to warm, humid conditions (Satyanarayana et al. 2019). If the use of plastic bags cannot be avoided, then they should be left open. Air circulation prevents condensation and growth of superficial molds (Olsson et al. 1996).

3. Liquid nitrogen, ice, Silica gel

Some soil samples need to be preserved at the field to prevent deterioration and DNA of microfungi in soil samples can be preserved at the field with liquid nitrogen (Graham 2001, Frey- 
Klett et al. 2005). If the distance between the collecting site and laboratory is not very far, then ice can be used for this. Some specimens are directly sequenced without obtaining a culture and therefore, specimens are preserved in tubes with dried Silica gel at the field after sanitation and surface sterilization.

4. Global Positioning System (GPS) recorder and maps

It is important to record accurate latitude, longitude and altitude of the collecting site, as these data are important during deposition of the material (Fuhrer 2008).

5. Field notebook, pens, tags, plant guides

Collection details such as location, host/substrate, habitat, mode of life, collection date, collector, collecting number/temporary code are recorded. Plant or animal/insect guides need to identify the host or substrate (Shepherd \& Totterdell 1988). All collecting details are noted on tags assigned to the specimen.

6. Camera

Photography at the field is necessary to show the natural habitat of the fungi, disease symptoms and vegetation pattern of the location. Photographs are linked to each specimen by a unique reference number (Young 2005).

7. Hand lens and gloves

Hand lenses are useful to see the surface view of fruiting bodies at the field or to check whether samples have fruiting bodies (Leonard 2010). When the collected samples are aquatic or marine, gloves can be worn to protect hands.

\section{Collection and storage}

Collecting fungi is not an easy task. The collected specimens are the representatives of the taxa. Therefore, over-mature, premature, or badly insect-damaged specimens are avoided because some characters are lost in damaged specimens (Hyde et al. 2010). Replication is important to confirm the accuracy of the size, availability and morphology of the fungal structures. In fact, some journals require more than one specimen and culture collection when describing a new species (Seifert \& Rossman 2010). If the collected specimens are taxonomically novel species, then the replicate samples may be designated as paratypes or syntypes (Dubois 2010). Duplicates should be deposited in different fungaria. However, if the material is limited, but taxonomic novelty is clear, then the author may be able to write a letter to editors and reviewers to confirm its novelty without depositing to different fungaria (Dubois 2010, Seifert \& Rossman 2010).

It is necessary to check the substrate carefully by a hand lens and cut or pick the substrate with fruiting bodies. If the fruiting bodies are immersed or erumpent in the substrate, it is difficult to see the fungal structures (Dai et al. 2018). However, some species produce long necks or papilla, which can be seen by hand lens. For plant pathogenic fungi, diseased samples should be collected such as leaf spots, rotten fruits or dried plant parts as well as photographed in situ (Hyde et al. 2019). All collected samples should be stored in suitable containers separately with the collection detail tag. Terrestrial samples can be air-dried to remove any surface moisture before transport or storage (Stone et al. 2004). However, changes in microfungi probably do not occur immediately following collection, but changes are rapid in basal fungi and other delicate, fleshy fungi (i.e. macro-Xylariaceae, some Hypocreaceae, aquatic hyphomycetes). It is essential that samples are handled carefully and processed as quickly as possible. An archetypal fungarium specimen includes every part of the fungus to verify the complete morphology (Prance \& Fechner 2017). Sometimes, it is possible to see a number of individual fruiting bodies, which are at different stages of maturity with both sexual and asexual morphs together (Senanayake et al. 2017a). Generally, it is best if collecting is carried out in the morning. If samples are collected when the environment is misty, specimens absorb a lot of moisture thus making them easy to deteriorate (Halling \& Mueller 2005).

\section{Data to be recorded in the field}

Important morphological, ecological and physiological data need to be noted in the field (as shown in Fig. 2). Others should be photographed, such as disease symptoms and larger fruiting 
bodies. Macroscopic characters and some measurements need to be recorded from fresh material. Ecological data including locality, latitude/longitude, habitat, mode of life, disease symptoms, host, substrate, collector and collection date should be noted on site. The Global Biodiversity Information Facility (GBIF) which is one of the most functional taxon databases has listed a set of data that should be collected. However, these do not always apply to fungi and it is important to refer GBIF (https://www.gbif.org/) when appropriate.

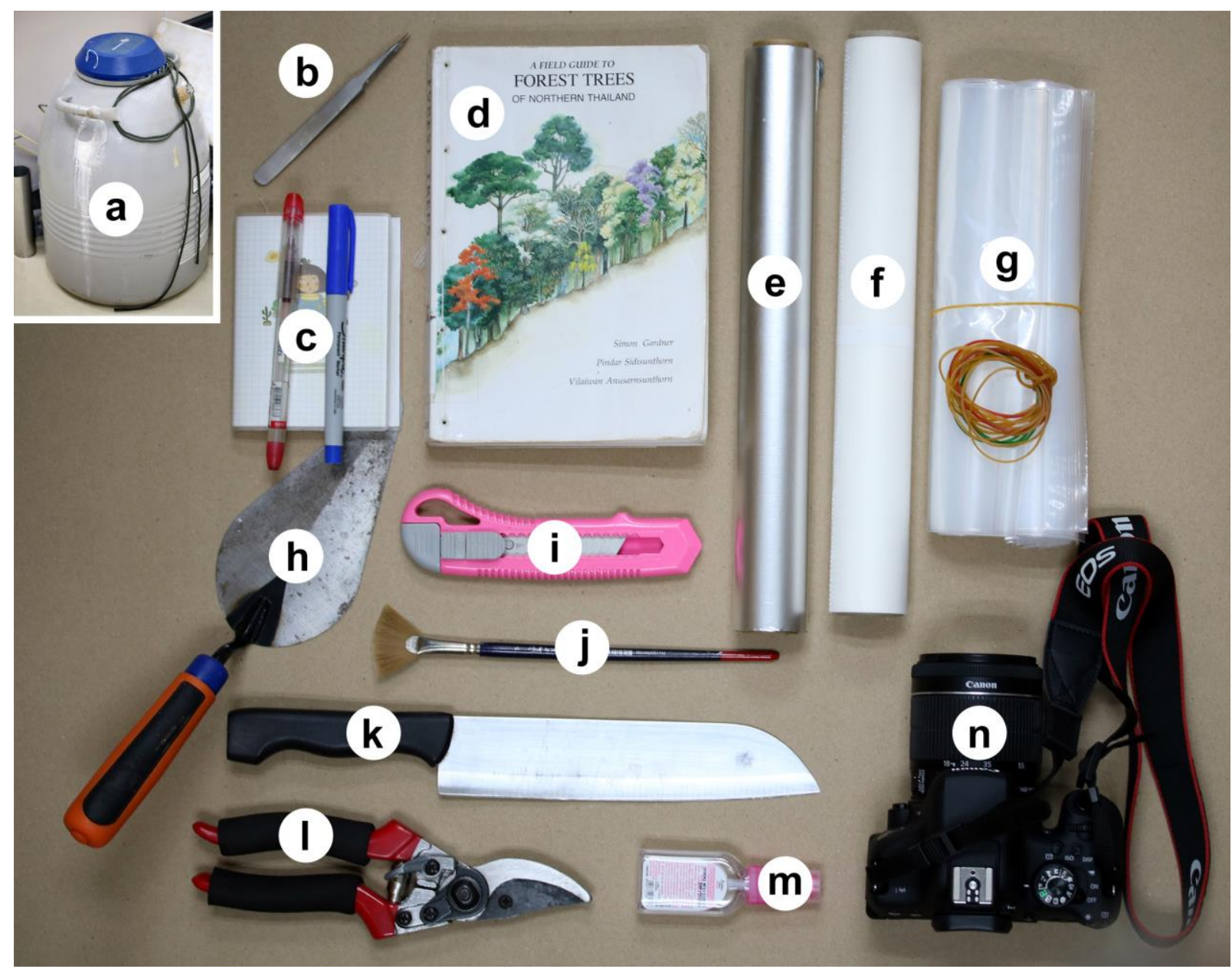

Figure 1 - Field tools generally used to collect the fungal specimens. a Liquid nitrogen. b Forceps. c Field notebook and pens. d Plant guides. e Aluminum foil. f Water-proof paper bag. g Zip-lock polythene bags and rubber bands. h Trowel. i Cutter. j Soft paint brush. k Pocket knives. 1 Metal snips. m Insect repellent spray. n Camera.

A temporary reference number is assigned for each specimen along with the above details. Temperature, humidity, GPS data and vegetation pattern are noted, when necessary. All macroscopic photographs, such as habitat, host, substrate and fruiting bodies in the case of macrofungi should be taken at the field and linked to the temporary reference number (Leonard 2010).

\section{Temporary storage}

When specimens reach the laboratory, they are stored temporarily in suitable containers to prevent deterioration. It is important not to bring specimens directly into the laboratory. Specimens may have associated insects, mites, nematodes and their eggs (Largent 1986). If specimens are directly brought to the laboratory, these can easily spread and destroy fruiting bodies and cultures. Specimens are cleaned carefully to remove soil, plant debris, insects and their eggs by a soft paint brush (Hosaka \& Uno 2011) and they are air-dried if wet. Specimens are stored in paper bags with 
collection details. If specimens are aquatic, then mud and soil can be removed by washing with water. However, this is not suitable for hyphomycetes and superficial fungi.

\section{Center of Excellence in Fungal Research}

Mae Fah Luang University, 333 Moo. 1

Thasud, Muang, Chiang Rai 57100, Thailand

\section{Fungal specimen collection form}

\begin{tabular}{|c|c|c|}
\hline Project code: SZU02 & $\begin{array}{l}\text { Project name: Taxonomy, phylogeny } \\
\text { and biogeographical distribution of } \\
\text { ascomyceteous fungi on monocotyledons }\end{array}$ & Date:25/02/2019 \\
\hline \multicolumn{3}{|c|}{ Location: Mushroom Research Center, Pa Pae Mae Taeng District, Chiang Mai } \\
\hline $\begin{array}{l}\text { Temporary code: } \\
\text { S3-11 }\end{array}$ & \multicolumn{2}{|l|}{ Taxon name (provisional): Anthostomella $\mathrm{sp}$} \\
\hline $\begin{array}{l}\text { Collector: I. C. } \\
\text { Senanayake }\end{array}$ & $\begin{array}{l}\text { Host \& substrate: dead stem of } \\
\text { Bambusa vulgaris Schrad. }\end{array}$ & $\begin{array}{l}\text { GPS: N19¹7.123' E } \\
98^{\circ} 44.009^{\prime} \text {, elev. } 900 \mathrm{~m}\end{array}$ \\
\hline Humidity: 73 & Temperature: $32{ }^{0} \mathrm{C}$ & \multirow{2}{*}{$\begin{array}{l}\text { Vegetation: rainforest } \\
\text { dominated by } \\
\text { Castanopsis } \\
\text { armata } \text { and Pinus } \\
\text { kesiya. }\end{array}$} \\
\hline $\begin{array}{l}\text { Soil texture: Soil dry } \\
\text { crust, inside moisten, } \\
\text { dark brown }\end{array}$ & Identified by: I. C. Senanayake & \\
\hline \multicolumn{3}{|c|}{ Notes: Most fruit bodies are over-matured and specimen is very dry. } \\
\hline
\end{tabular}

Figure 2 - Important details in a model data recording form.

\section{Incubation and succession}

Fungal succession has been defined as the sequential occupation of different fungi or different associations of fungi on the same substrate or site (Rayner \& Todd 1979). This happens because of a sequence of sporulating fungi on a substrate by mycelium. However, replacement of one fungal species by another is not necessary and some fungi are sporulated together on a substrate (Hyde \& Jones 2002). Sometimes, incubation of fresh specimens is necessary to obtain maximum fungal diversity, especially rare or slow growing species and it may increase the maturity and amount of fruiting bodies.

Specimens are subjected to surface sterilization with $70 \%$ ethanol, if necessary. Then, a piece of sterilized, moistened tissue paper or cotton wool is placed in a sterilized plastic box (Hyde \& Jones 2002). Specimens are held on two sterilized glass rods which are placed inside the box parallel to each other and this is incubated at room temperature with light. Continuous daily observations can grab the slow growing fungal species (Hyde \& Jones 2002). However, the major problem of incubating specimens in moist chambers is contamination from other fungi or bacteria. Therefore, it is necessary to avoid contamination and enhance the formation of fruit-bodies of slow growing fungi. Basic guidelines to avoid contamination are listed below.

1. All containers and tools used for moist chambers should be sterilized and transparent in order to examine fungal succession clearly.

2. A 12 hour light and 12 hours dark cycle are preferred in order to mimic natural conditions. 
3. Containers are slightly moistened with sterilized water and excess water may form rotting or deterioration of specimens and fungi, while less water may lead to dryness without fruiting body formation.

4. Specimens are surface sterilized if necessary, to remove soil, insects and their eggs.

5. Specimens are incubated at temperatures lower than the collection locality. According to our experiments $18-25^{\circ} \mathrm{C}$ gives a good yield of fruiting bodies and also less contamination. Air borne, soil borne fungi and other fast growing fungi on the specimens grow rapidly as the temperature increases. Therefore, moderately low temperatures facilitate the slow growing fungi.

6. Specimens are examined under aseptic conditions created on sterilized working benches (Fig. 3) between two flaming lamps. Daily examination yields a large number of species.

\section{Morphological observation at the laboratory}

Examination of morphology and illustrations of specimens are the key steps of a suitable protologue. With the improvement of optics technology, there are numerous modern microscopes with high contrast and magnification (Ahmad \& Ahmad Khan 2012). Additionally, various illustration techniques and software programs provide extra tools for obtaining detailed descriptions. Herein, conventional procedures, mistakes and suggestions to avoid potential drawbacks are explained.

\section{Microscope characterization}

Microscopy is the key technique to obtain morphological characters and cellular structures except for some macroscopic characters, which are visible to the unaided eye (Ahmad \& Ahmad Khan 2012). Different microscope techniques interpret characters in different ways. There are several microscopes available for fungal morphological studies that include classical light microscopy, electron microscopy, fluorescence microscopy, phase contrast microscopy, confocal laser scanning microscopy and atomic force microscopy. However, compound light microscopy is the most common way to use to observe the morphological characters. Microscopes should be cleaned and maintained on a regular basis. This is important to obtain clear pictures of appropriate contrast and prevent malfunction.

\section{Pretreatment of fungal specimens}

Fungal structures should be optimally mature in order to observe using a microscope. There should also be sufficient fruiting bodies and/or other structures for examination. If a fungarium or a dried specimen is very dried, it is necessary to rehydrate before the examination (Senanayake et al. 2018). A small piece is separated from the specimen and rehydrated directly by placing a drop of sterilized water, potassium hydroxide $(\mathrm{KOH})$, ammonium hydroxide $\left(\mathrm{NH}_{4} \mathrm{OH}\right)$ or covered with sterile wet tissues (Foster et al. 2011). Since some species chemically react with $\mathrm{KOH}$ or $\mathrm{NH}_{4} \mathrm{OH}$, hydrating a tiny piece of the specimen for morphological examination is recommended.

\section{Preparation of fungal structures for microscope examination}

\section{External morphological character examination}

Fruiting bodies or vegetative structures of the fungi, their niche characters and colony surfaces at low magnification are essential for a better understanding of the fungi (Gupta et al. 2013). Stereomicroscope is an indispensable instrument used to observe the characters and has a magnification in the range 10-100 times that of the object. However, hand-lenses are used in the field to determine the availability of fruiting bodies. External characters of fruiting bodies, conidiophores, conidial formation and their orientation on the substrate or in culture can also be examined (Fig. 4). In addition, the position of fruiting bodies on the substrate, symptoms and other interactions with the niche mycota are examined prior to the internal character examination. 

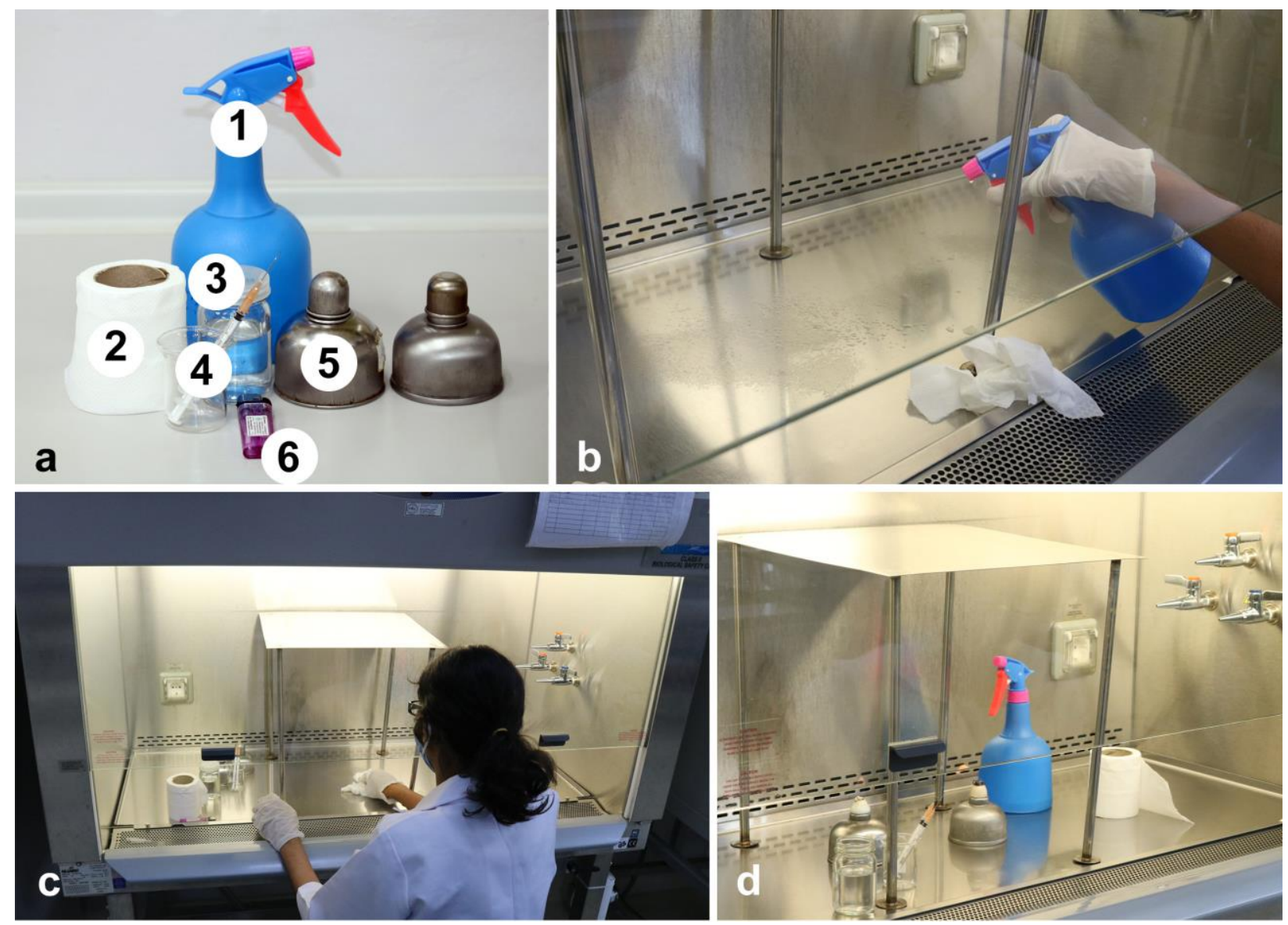

Figure 3 - Aseptic working bench. a (1) 70\% alcohol sprays, (2) Tissues, (3) 70\% alcohol bottle for dipping, (4) Needles, (5) Alcohol lamps. (6) Lighters. b, c Surface sterilization of working bench with $70 \%$ alcohol. d Common set-up of materials inside the laminar flow.

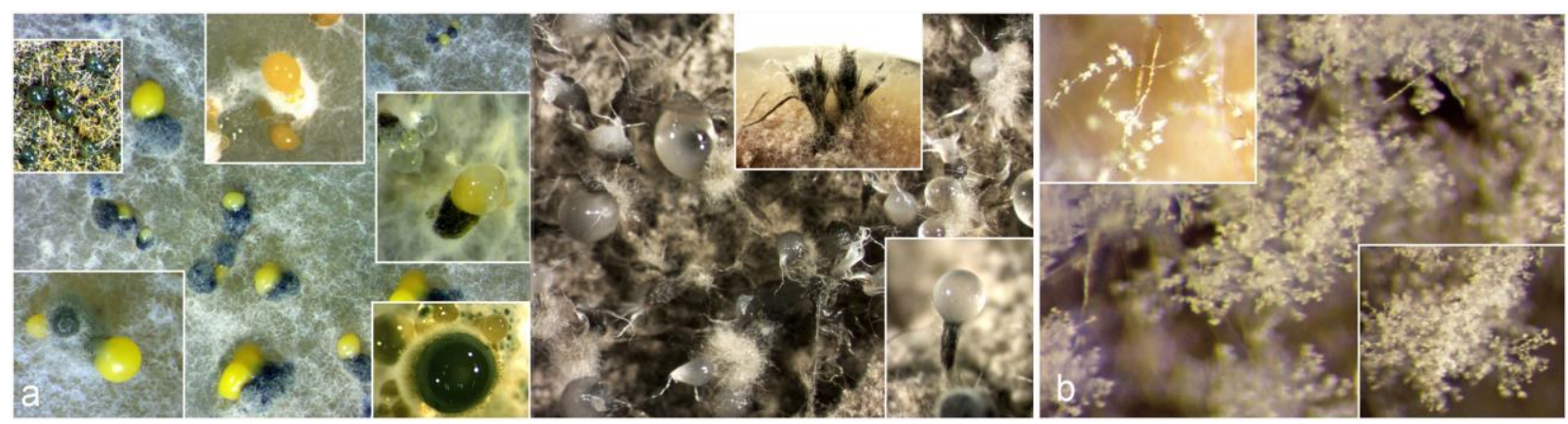

Figure 4 - Photographs of fruiting bodies and conidial orientation from stereomicroscope. a Conidiomata on cultures. b Conidial attachments and orientation of hyphomycetes on cultures.

\section{Sectioning}

Vertical or horizontal sections of ascomata or conidiomata give essential taxonomic information including stromatic characters, shape and size of fruiting bodies, position of fruiting bodies in host tissues and ostiole or peridium characters (Foster et al. 2011). Sections can also help to establish the arrangement of paraphyses and pseudo-paraphyses and distinguish cellular pseudoparaphyses from tubular pseudo-paraphyses. We can also see how a species colonizes a substrate, e.g. foliar epiphytes or tar spots (Foster et al. 2011). Sectioning can be done by free hand or by freezing microtome. Basic guidelines for both ways of sectioning are discussed. In our experience, the best sections are freehand although it takes some time to become skillful (Smith et al. 1981). 
Microtome sections are often too thick to establish cell wall structures, even when set at less than $10 \mu \mathrm{m}$.

\section{Freehand sectioning}

Double or single-edged razor blades, slides, coverslips, sterile water or any mounting reagent, needles, stereomicroscope and the material needed to be sectioned are prepared for the freehand sectioning (Fig. 5). Fresh or remoistened specimens are used for sectioning. Material is held between the thumb and index finger of one hand (Senanayake et al. 2014) and the razor blade which is held on the other hand is drawn across the material with the edge outward from the operator. The blade slices the fungal material and this process is repeated several times until a thin section is obtained (Gupta et al. 2013). A needle dipped in the water is used to pick up the sections from the specimen directly if the sections do not stick to the needle. All sections are placed in drops of sterile water or mounting reagent. If the material is too small or delicate, it can be placed in a slit or pith made of plasticine or blue-tack to hold it firmly. The razor blade becomes blunt after taking several sections and should be changed often (Mukerji \& Manoharachary 2010). There are several guidelines to obtain good sections.

1. Excess amount of the hydrating agent is blotted by tissues to prevent sticking sections to the specimen.

2. If the hydrating agent is not water, it is necessary to pre-examine the reaction of these reagents with a small piece of the specimen. Some reagents chemically react with the stromatic tissues of the fungi such as stromatic tissues of Cryphonectriaceae species which turn purple-blue when reacting with $\mathrm{KOH}$.
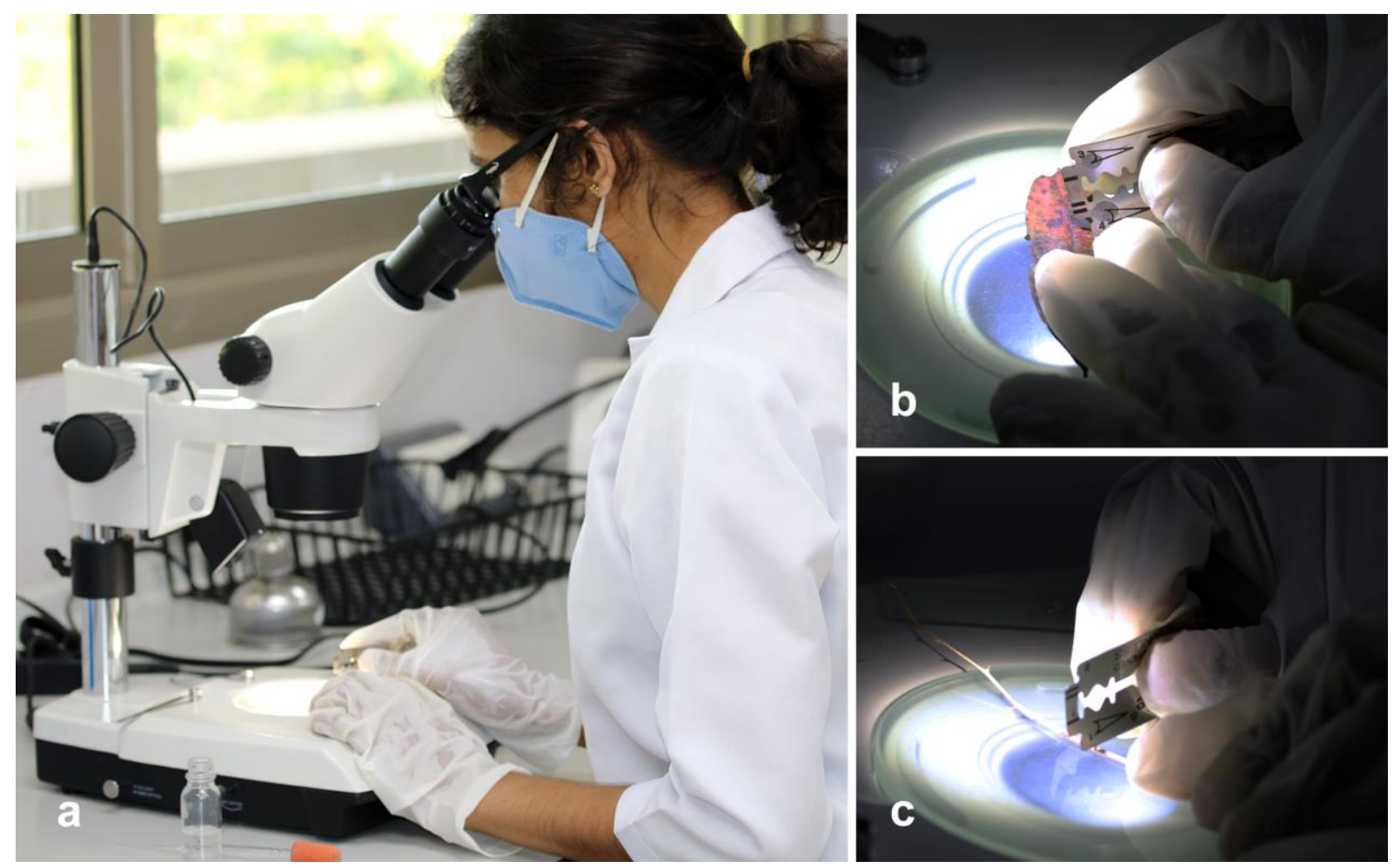

Figure 5 - Free hand sectioning using a stereomicroscope. a Observation of fruiting bodies with stereomicroscope for sectioning. b, c Sectioning of fruiting bodies in different substrates (b) A leaf. (c) A twig.

\section{Microtome sectioning}

Microtomy is a technique which provides sections of frozen tissue for microscope examination and it is commonly used in hospitals for medical mycological studies (Gupta \& 
Pandey 2013). A microtome comprises three major parts; the tissue holder, the blade carrier, and adjustment screws with feed wheels that line up the tissue in the correct position to the blade and advance the tissue for successive sections (Fig. 6). The stage cabin includes a blade and tissue holders which freezes to lower than $-20^{\circ} \mathrm{C}$. The specimen is placed in a drop of solidifying reagent on the tissue holder and the thickness of sections is set by adjusting the advancement device (Huhndorf 1991(. In most microtomes, sectioning begins by moving the sample over the blade. Sections are picked from the blade using a fine needle or by a fine paint-brush and it is placed in cryoprotectant tubes for storage. Alternatively, sections can be placed in a drop of mounting agent for immediate observation. Below are guidelines for obtaining good sections.

1. An optimal amount of sample is selected and placed in the solidifying fluid perpendicularly to the blade. If the sample is not placed in the correct plane, then the sections may not show distinctive characters.

2. A moistened brush is used to pick up the sections from the blade.

3. Sections are immediately placed in a drop of mounting reagent to prevent trapping air bubbles in the sections.
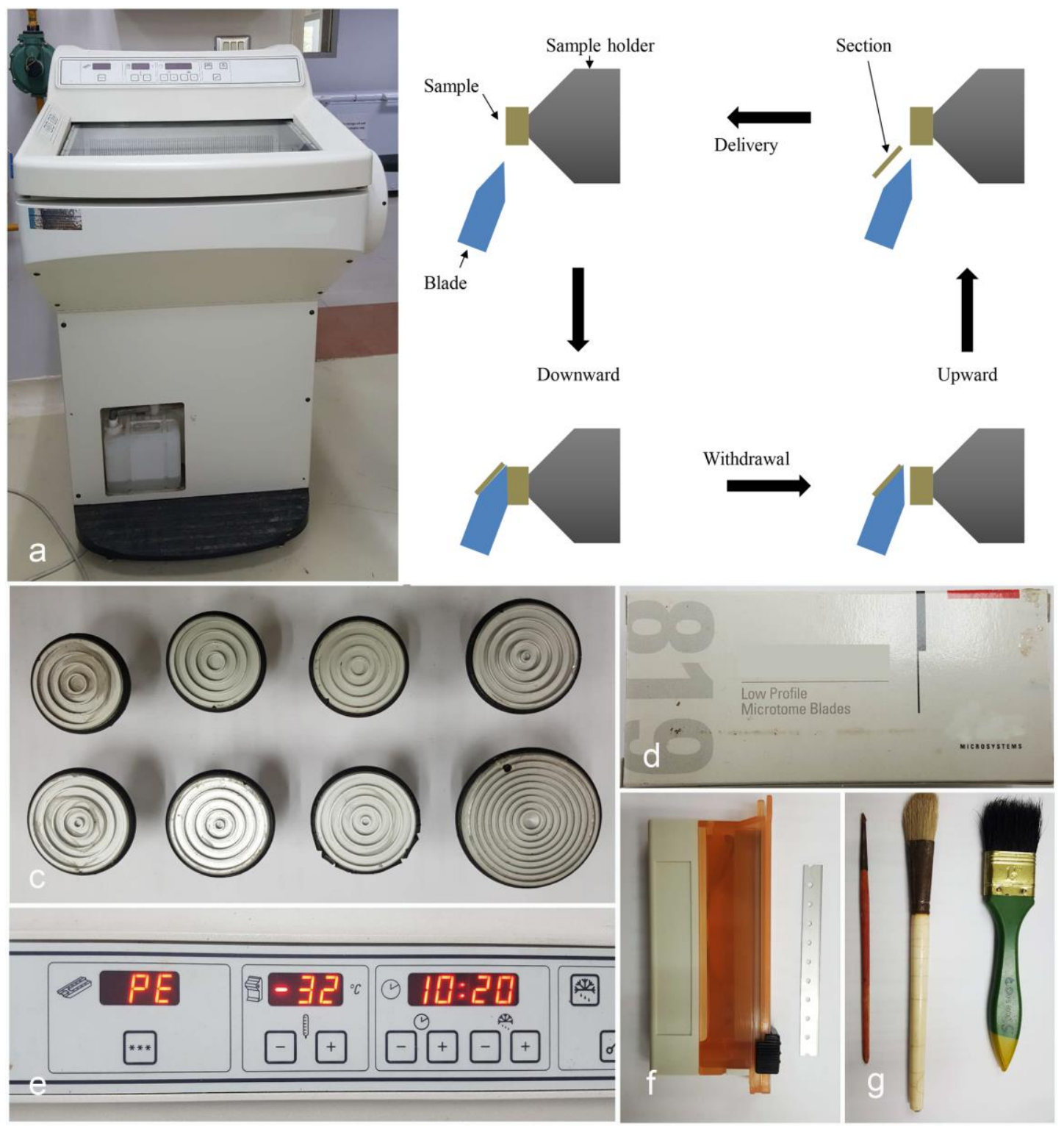

Figure 6 - Freezing microtome, mechanism and tools needed. a Microtome. b Illustration of the mechanism. c Specimen holders. d, f Blade box and blades. e Condition adjusting bar. g Brushes. 


\section{Mounting}

It is necessary to prepare microscope slides for observing fungi. Clean microscope slides, coverslips and recently prepared mounting regents are used (Huhndorf 1991(. Reagents and stains are stored in bottles and labeled. Fungal structures are arranged individually using a needle. The coverslip is placed carefully on the specimen and the excess amount of mounting reagent is blotted using a tissue paper while gently pressing (Gupta et al. 2013). Pressing is important to arrange fungal structures on the same plane (Vignesh et al. 2013).

All the morphological structures should be mounted in the same mounting reagent for photography. The mounting reagent should be mentioned as different mounting reagents have different contrast and viscosity. Images are very sharp when the refractive index of the mounting regent is low. Water is the best mounting reagent as it gives more neutral and natural photographs. Water keeps structures in the same plane in photography. $\mathrm{KOH}$, glycerol and stains are used when needed. $\mathrm{KOH}$ gives more rigidity and it is important to mount in $\mathrm{KOH}$ when the specimen is too dry. However, sometimes $\mathrm{KOH}$ reacts with fungal structures, such as stromatic tissues of Cryphonectriaceae which turn to purple. Glycerol keeps fungal structures without drying for a long time.

\section{Squash mounts}

This is the very basic mounting method for quickly observing taxa or for examining characters such as the appearance of stromatic tissues, surface view of ascomata, characters of peridium and papilla, tissues of hamathecium, conidiophores and conidiogenous cells, asci, ascospores or conidia and conidial ontogeny and vegetative hyphae (Gadoury \& MacHardy 1982).

A drop of mounting agent is placed on a clean slide and a few fruiting bodies are placed in a drop of mounting agent. If the fruiting bodies are large, then they are cut horizontally to obtain the internal structures. Then fungal mass is picked by a needle or fine forceps (Gadoury \& MacHardy 1982). If the fungal specimen is a hyphomycetous taxon, then the moistened needle tip is dragged over the conidia to retrieve them (Gadoury \& MacHardy 1982). If the slide is crowded with fungal or host plant tissues, then the excess plant or fungal tissues are removed using a needle. There are several guidelines to obtain good squash mounts:

1. It is important to use a small amount of fungal structures otherwise it will be too crowded for photography and hard to examine the structures clearly.

2. Add a few drops of $20 \%$ alcohol to separate fungal hyphae.

3. If the fungal structures are too dry, then add $10 \% \mathrm{KOH}$ to make them rigid.

4. The fungal structures may be destroyed if the coverslip squash is too hard.

5. Gently heating can be used to remove air bubbles.

\section{Transparent adhesive tape (Sellotape) mount or tape-lift mounts}

This technique results in very little disturbance of fungal structures and is useful for identification and taxonomic work (Harris 2000). Air bubbles trapped within tissues due to the insertion of transparent adhesive tape is a disadvantage (Onions et al. 1981) and this affects the interference patterns during differential interference contrast (DIC) microscopy. Adhesive tapes, which dissolve in mounting reagent or adhesive tapes, which do not trap the mounting agent within tissues are available. However, these kinds of tapes are expensive or have weak adhesiveness (Fairclough et al. 1985).

A piece of transparent adhesive tape $(<2 \times 2 \mathrm{~cm})$ is gently placed on sporulating species or culture. The tape is gently pressed until fungal structures stick and the tape is removed using fine forceps. The tape with the attached fungal structures is placed on a drop of the mounting agent on the slide and observed with a microscope.

\section{Slide culture mounts}

This technique (Fig. 7) allows for observing the mycelia, development of fruiting structures, spore germination and conidial sporulation and preserving the fungi in a relatively undisturbed state 
(Riddell 1950, Cai et al. 2009). This method allows mycelia to grow and sporulate on the microscope slide (Liu et al. 2011). The ordinary slide culture mount is performed with a microscope slide placed on two parallel glass rods in a sterile moisten Petri-dish ( $\mathrm{Su}$ et al. 2011, 2012). A block of agar is placed on the microscope slide and the fungus is inoculated by a sterile needle. A sterile coverslip is placed over the agar block and is slightly pressed to ensure adherence. The apparatus is then incubated at $20-25^{\circ} \mathrm{C}$ (Rosana et al. 2014). In addition, this mount allows for storing slides without drying for a long period. Fungal structures embedded in the agar also can be observed.

\section{Direct culture on slanted agar plate}

This method allows growing a fungus on a thin agar slant on a Petri-dish and observing it directly on the upper side of the plate (Sanders 2012). Approximately, $5 \mathrm{~mL}$ of agar is spread on a sterile Petri-dish $(60 \times 15 \mathrm{~mm})$ and a slant is made. Otherwise, it may be difficult to observe the colony, if the medium layer is too thick. The fungus is inoculated onto the plate and fungal growth can be easily observed (Sanders 2012). Culture media should be colourless, such as water agar or $10 \%$ PDA. It may be possible to mix fungal conidia or spores with media before pouring. However, the media should be at a low temperature. Otherwise, spores or conidia may lose their viability.

\section{Film-culture mount}

This method deals with growing a fungus in a thin film $(<0.2 \mathrm{~mm})$ of media on a microscope slide (Hill 1996). In addition, film-culture mounts facilitate sporulation and direct examination of fungal structures with minimum disturbance (Murray et al. 1995). The thin agar film also limits excessive mycelial growth, while it often promotes sporulation.

Small glass Petri-dishes $(60 \times 15 \mathrm{~mm})$, glass rod pieces, microscope slides $(46 \times 27 \mathrm{~mm})$, coverslips, syringe, filter papers $(55 \mathrm{~mm} \times 15 \mu \mathrm{m})$, needle, forceps, sterilized water and culture media are required. Two small glass rods are placed parallel to each other on a moistened filter paper placed at the bottom of the Petri-dish. Then, a microscope slide is placed on two glass rods and this whole apparatus is sterilized. A few drops of culture media are spread on the slide to make a thin film using a sterile glass spreader and this agar-film is then inoculated with the fungus. The Petri-dish with the inoculated slide is covered with the lid and incubated at an appropriate temperature (Fig. 8). When the fungus grows and sporulates on the agar film, the microscope slide is examined directly or covered with a coverslip.

\section{Broth-culture or cavity mounts method}

This is a mounting and culturing method introduced herein and is mainly based on growing a fungus in a small amount of agar broth in a microscope cavity slide. This simple apparatus facilitates growth of the fungus with a minimal amount of media, observation directly with minimum disturbance and saves time.

Small glass Petri-dishes $(60 \times 15 \mathrm{~mm})$, glass rod pieces, microscope cavity slides $(46 \times 27$ $\mathrm{mm})$, coverslips, a syringe, filter papers $(55 \times 15 \mu \mathrm{m})$, needle, forceps, sterilized water and culture media broth are required. A filter paper is placed at the bottom of the Petri-dish and two small glass rods are placed on it. Then, a microscope cavity slide is placed on two glass rods. The apparatus is sterilized and the filter paper is then moistened with sterile water. A few drops of agar broth are placed in the cavity slide and are then inoculated with the fungus. The Petri-dish with the inoculated cavity slide is covered with the lid and incubated at $20-25^{\circ} \mathrm{C}$ (Fig. 9). When the fungus grows and sporulates on the agar film, the microscope slide is examined directly or covered with a coverslip. This method is most suitable for motile spore producing fungi. 


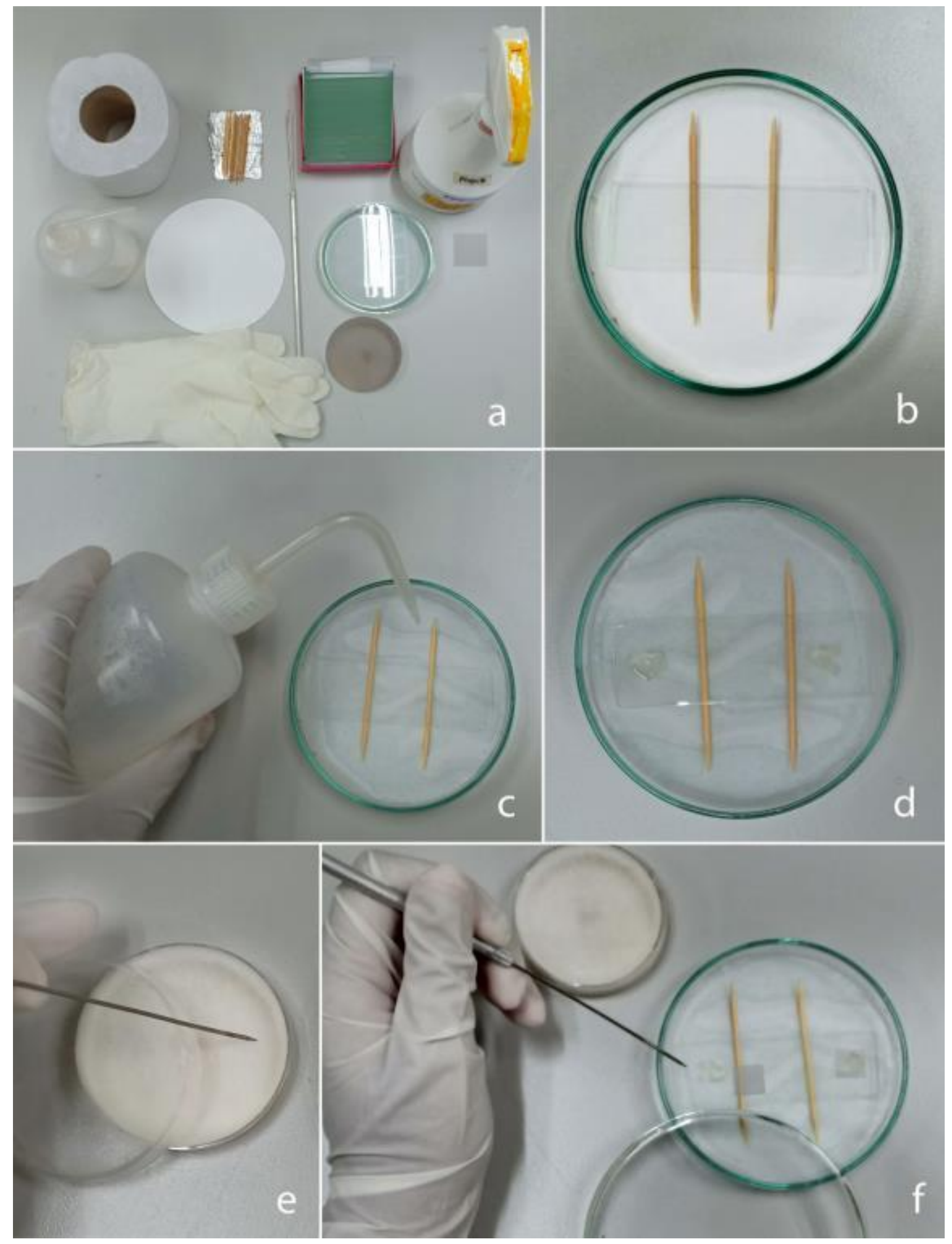

Figure 7 - Apparatus for slide culture method. a Tools. b Sterilized filter paper, sticks and slides. c Adding sterilized water on filter paper to maintain humidity. d Potato Dextrose Agar (PDA) blocks on both sides of the slide. e Picking a small amount of mycelia. $f$ Inoculation of PDA blocks. 

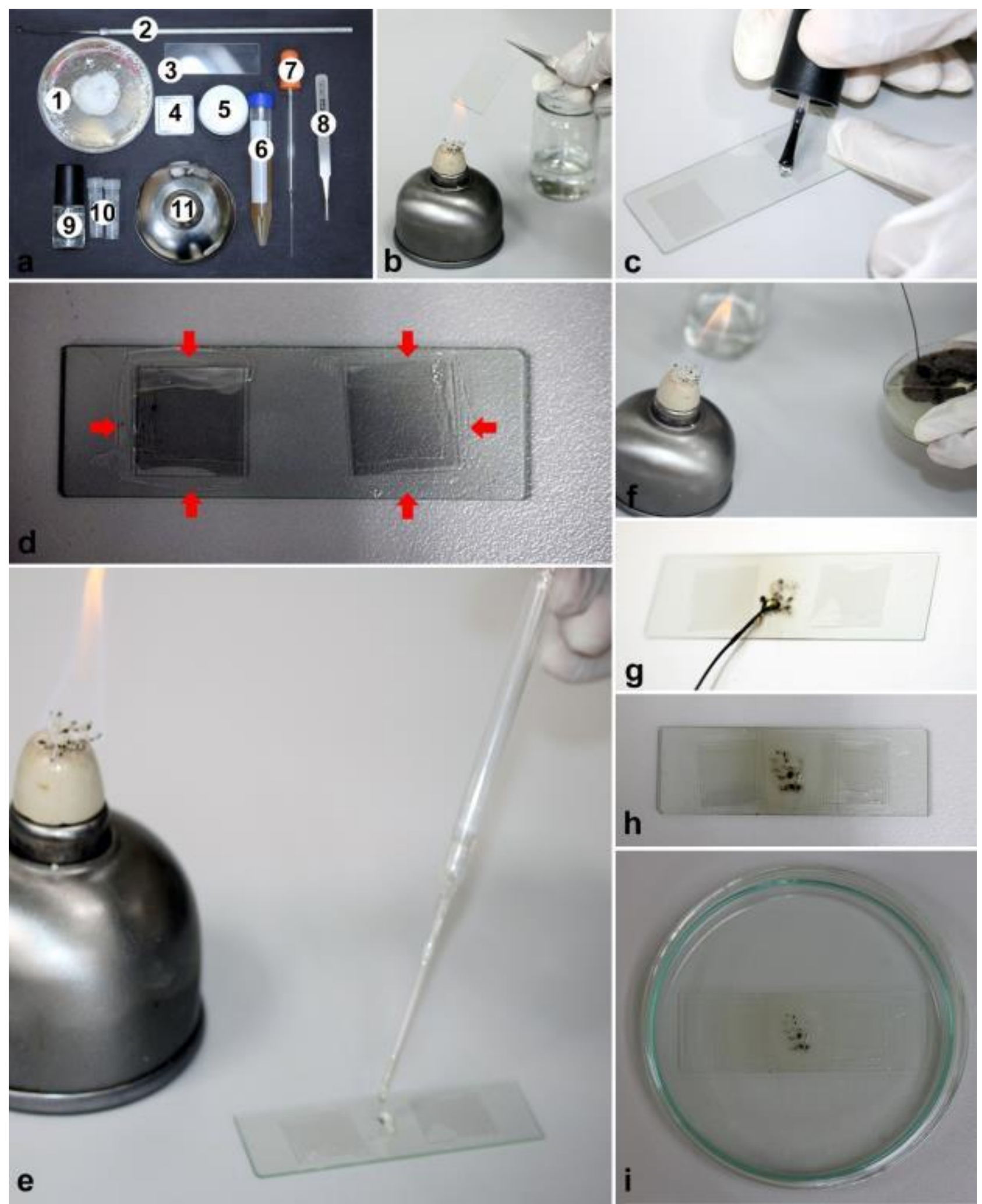

Figure 8 - Tools and process of film-culture mount method. a Materials (1) Pure fungal colony, (2) Inoculating loop, (3) Microscope slide, (4) Coverslips, (5) 70\% alcohol, (6) PDA, (7) Dropper, (8) Forceps, (9) Nail polish, (10) Sterile distilled water, (11) Alcohol lamp. b Microscope slide sterilized in $70 \%$ alcohol. c, d Sealing the coverslips using nail polish. e Placing a drop of PDA between the coverslips. $f$ Transfering of mycelia or spore using wetted inoculating loop into the PDA drop. g Spreading the mycelia or spores. h Placing coverslip. i Incubation the Petri-dish. 

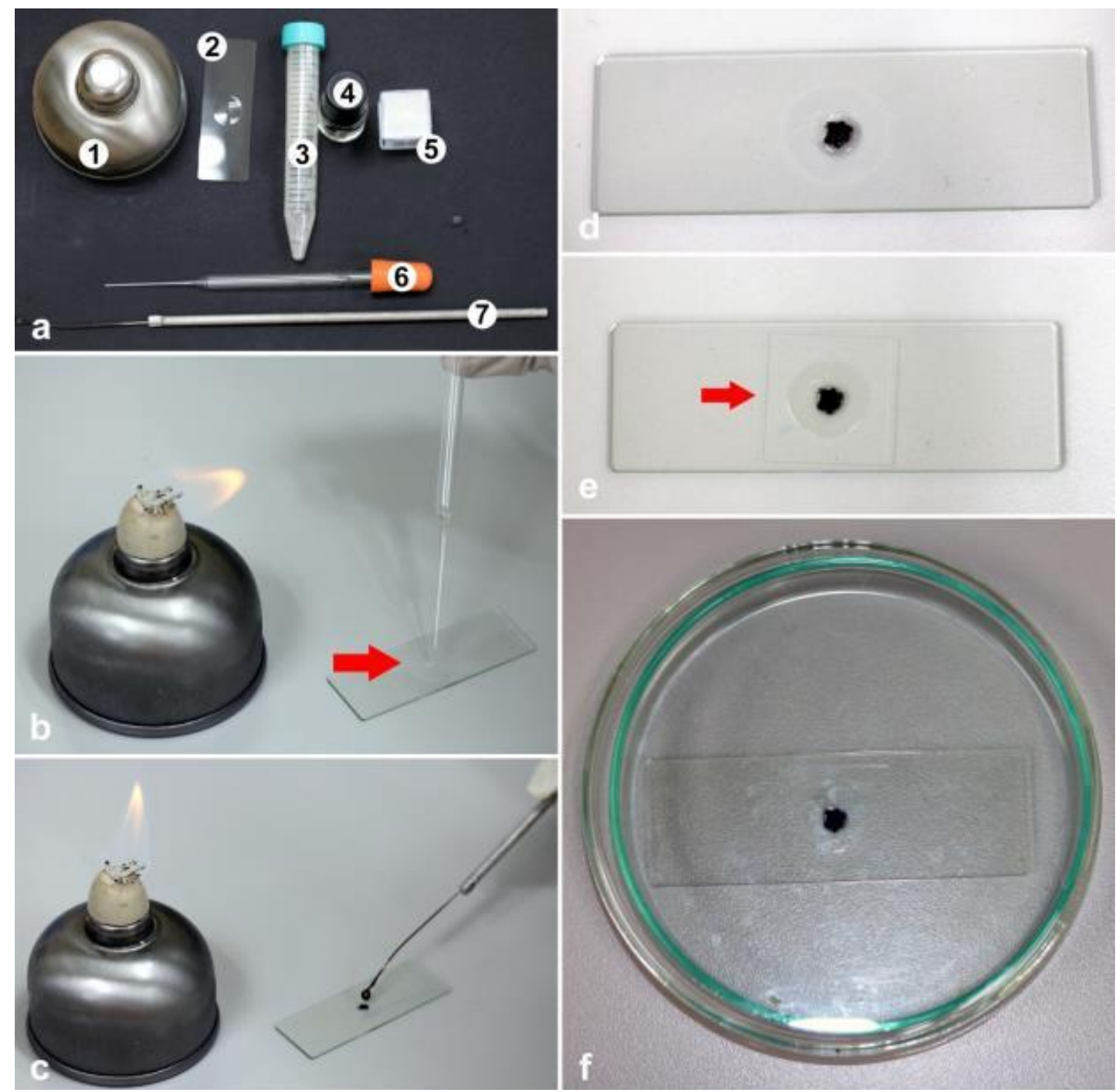

Figure 9 - Apparatus of broth-culture method. a Materials (1) Alcohol lamp, (2) Cavity slides, (3) PDA broth, (4) Nail polish, (5) Coverslips, (6) Pasteur pipette with dropper, (7) Inoculating loop. $b$ Placing a drop of PDA broth on a microscope cavity slide (arrow). c Inoculating the drop of PDA broth. d, e Microscope cavity slide and enclosed with a coverslip. f Incubation.

\section{Staining}

Microscope slides can be stained when necessary. Staining helps to obtain more contrast and sharper structures (Adds et al. 1999, Karp 2009). Lacto-phenol cotton blue is best to stain fungal structures. However, once tissues are left in the stain for a few minutes, then excess stain is washed away by a drop of ammonium hydroxide or distilled water (Santana et al. 2018). These reagents are applied to one side of the coverslip and a tissue paper is placed on the opposite side to absorb this ammonium hydroxide or distilled water (Isenberg 2007). However, it is important to avoid moving the coverslip to prevent physical damage to the fungal structures. There are many stains used for specific reactions (Smith 2019). Commonly used chemical reagents and their reactions on different fungal structures are listed below (Table 1).

\section{Preparation of permanent microscope slides}

Some slides with the fungal structures represent the type specimens of the species and therefore, sometimes microscope slides can be kept for a long time (Foster et al. 2011). Kohlmeyer 
\& Kohlmeyer (1972) proposed a simple method to make permanent microscope slides for fungi. There are several steps in this process such as fixation, dehydrating, clearing and mounting.

Table 1 Common chemical reagents used in fungal taxonomy and their reactions.

\begin{tabular}{|c|c|c|}
\hline Stain/reagent & Fungal structure & Reaction \\
\hline Water & Any fungal structures & Rehydrate the structures \\
\hline $\mathrm{KOH}(3 \%)$ & Any fungal structures & Rehydrate the structures \\
\hline $\mathrm{KOH}(10 \%)$ & $\begin{array}{l}\text { Stromatic tissues (e.g. Hypocreaceae, } \\
\text { Cryphonectriaceae) }\end{array}$ & Turn to purple \\
\hline $\mathrm{NaOH}(10 \%)$ & Fungal cell wall & React with Chitin and give clearer structures \\
\hline $\begin{array}{l}\text { Lacto-phenol cotton } \\
\text { blue }\end{array}$ & Cyanophilic spores & Spore walls stain blue \\
\hline \multirow[t]{3}{*}{ Melzer's reagent } & Amyloid spores or hyphae & Stain blue to black \\
\hline & Pseudoamyloid spores or hyphae & brown to reddish brown \\
\hline & Inamyloid spores or hyphae & Faintly yellow \\
\hline Congo red & Hyphal wall and cytoplasm content & Stain reddish pink \\
\hline India ink & Spore sheath & $\begin{array}{l}\text { Sheath remains white, background stains } \\
\text { black }\end{array}$ \\
\hline \multirow[t]{2}{*}{ Lactoglycerol } & Any fungal structures & Remove excess stains \\
\hline & & Provide a clear mounting medium \\
\hline Schultze's reagent & Amyloid spores or hyphae & Stain blue to black \\
\hline Baral's regent & Amyloid spores or hyphae & Stain blue \\
\hline Aniline blue & Endophytic hyphae in the meristem & Stain blue \\
\hline
\end{tabular}

Live fungal tissues are killed rapidly by precipitating the proteins during the fixation (Glime \& Wagner 2017). The most commonly used fixatives are 70\% alcohol, Bouin's fluid and formalin. Dehydration helps to remove the excess water and allows complete infiltration of tissues with the sealing agent. The dehydrogenase enzyme has been used in Kohlmeyer \& Kohlmeyer (1972). If the slide does not dehydrate properly, then tissues appear as an opaque mass. However, rapid dehydration may distort and shrink the tissues and therefore, gradual dehydration is recommended (Glime \& Wagner 2017). Clearing is the next step, which helps to remove all the traces and enhance the infiltration of the tissues with a mounting agent. Xylene is the commonly used clearing agent (Connell \& Padgett 1988). Then, a few drops of mounting agent such as glycerin are placed on the specimen and a clean coverslip is slowly placed on the mounted specimen. The slide is examined using a compound microscope to confirm whether all the characters are nicely preserved or not. Then, the coverslip is gently pressed to remove excess glycerin and a tissue paper is used to blot them. Finally, all the edges are sealed with a colourless nail polish (Kohlmeyer \& Kohlmeyer 1972). Once the process is complete, the slides are thoroughly dried and stored in a slide storage box with the dry, low humid condition.

The double-coverslip method is another permanent slide preparation technique which was originally introduced by Diehl (1929). This method has been used for preserving voucher specimens and type material where slides are needed. Daghighi et al. (2016) has proposed a method for permanent slide preparation of soil fungi.

\section{Photography, arranging photomicrographs and illustrations}

Photography is very important for morphological characterization. It is essential to obtain high-quality digital images and the quality of photographs depends on the microscope, camera and operator (Braddock 2000). A poorly configured microscope produces substandard images, even when the digital or conventional cameras are excellent. Besides, some imperfections that are not immediately visible when looking through the microscope eye-piece, can be revealed by the digital imaging system (Delly 1988). Both line drawings and photomicrographs are used to illustrate the fungi. Hooke (1665) published the first known illustration of fungi (Fig. 10). 


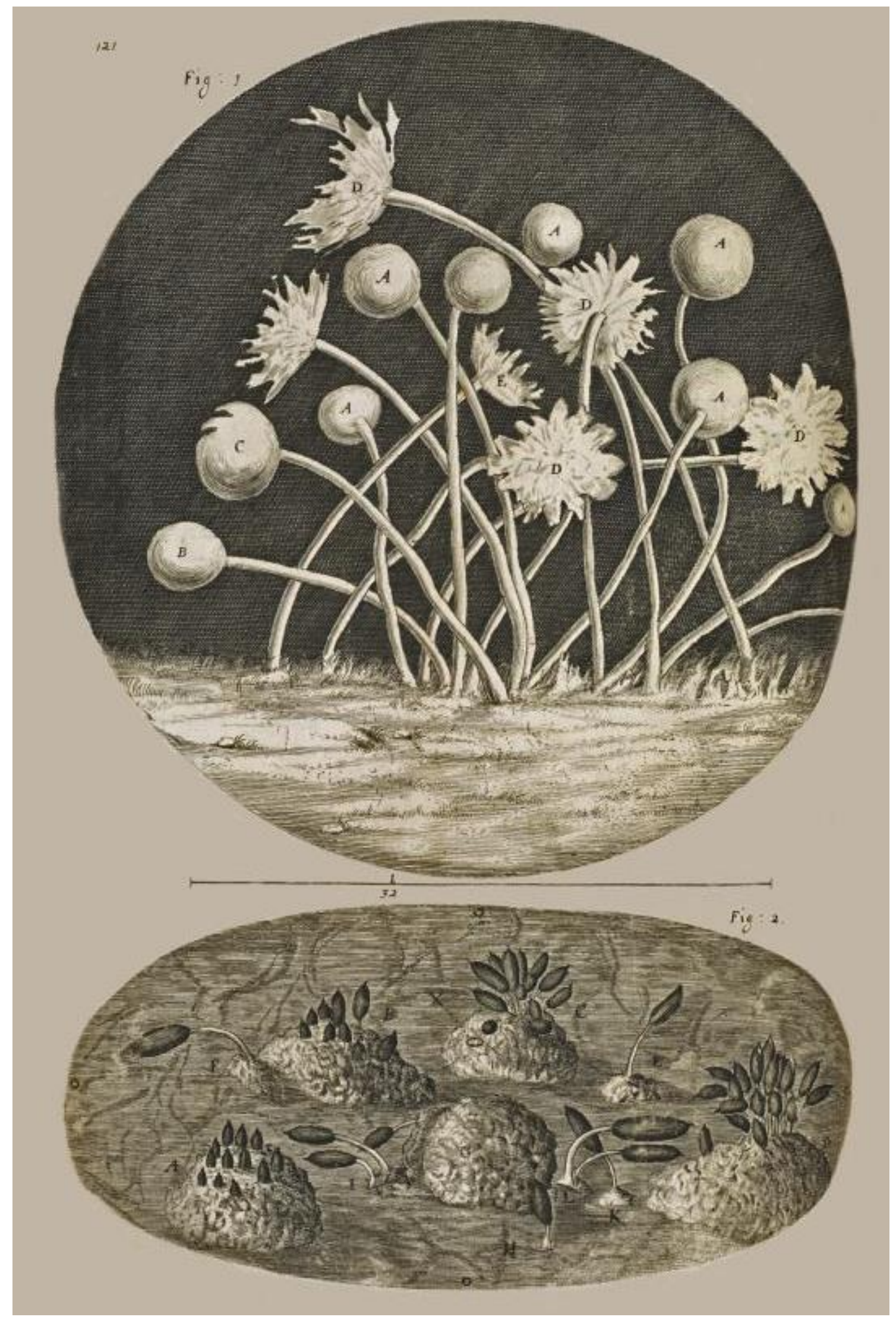

Figure 10 - The very first micrograph of fungi: "Blue mold and its vegetative parts arising from putrefaction" (Photograph referred from Hooke 1665, page 121).

A digital illustration method for microfungi has been proposed by Barber \& Keane (2007) and the original scanned images are used as basic electronic shapes which can be processed into 
complete figures later. Almeida \& Gusmao (2015) have presented a further modified version of this method. Original figures and illustrations are sometimes redrawn by tracing over the viewed image onto a paper (Senanayake et al. 2018). Additional stippling of the characters such as the pigmentation, shape, and ornamentation are added (Wijayawardene et al. 2016) and the scanned images are then edited and combined to make photoplates.

Photomicrography is the most commonly used descriptive illustrating method. This is mainly based on photographs taken in the field, photographs were taken using a stereomicroscope, compound or higher resolution microscope and photographs taken using a digital camera together with line-drawings (Silverman 1987). Several software programs can be used for photomicrography, such as Adobe Creative Cloud (Adobe Systems, USA), Adobe Illustrator (Adobe Systems, USA), Adobe InDesign (Adobe Systems, USA), Adobe Photoshop (Adobe Systems, USA) and Corel Draw (Corel, Canada). These software programs give various tools to enhance picture quality and show the characters clearly (Mancini \& Sidoriak 2017).

However, it is important to take several good photographs of the same character and select the best shot. It is not recommended to edit them too much using software programs. These software programs help to arrange and scale the pictures as well. Both film and digital methods can be used to obtain photomicrographs. However, film photomicrography is not being used at present since digital imaging has been developed in the last few decades allowing images to be easily manipulated. Digital images are easily incorporated into other digital documents and can be exported to image analyses systems (Papagianni 2014) or posted on a website. They are also easy to copy, store and archive. Digital images are easy to annotate with appropriate software programs for presentations or archives (Barry \& Williams 2010).

Digital images are used to obtain qualitative and quantitative taxonomic information from fungi (Adams \& Thomas 1988). There are several additional software programs that can be used for the photomicrographs preparation such as Adobe Creative Cloud (Adobe Systems, USA), Adobe Illustrator (Adobe Systems, USA), Adobe InDesign (Adobe Systems, USA) and Adobe Photoshop (Adobe Systems, USA). All the mentioned software programs can help to arrange and scale photographs.

\section{What should be in a decent illustration or photomicrograph?}

Illustrations are part of the final output of different morphological structures to be used in the publication. Therefore, the inclusion of complete details in an illustration is necessary (Fig. 11). Below are guidelines to make a decent illustration:

1. It is better for both illustration and legend to be arranged on a single page. Therefore, it is necessary to fix the size of the illustration or photomicrograph first and then arrange the images as necessary. Generally, A4 is the most commonly used size in scientific publications (Fig. 11).

2. Photographs included in the illustration are selected first and they are edited as necessary. Photographs are arranged on the plate filling the space. There are a few aspects that one needs to bear in mind. Photographs are arranged in order from habitat or ecological characters, the external appearance of fruiting bodies, internal characters of fruiting bodies and colony characters. It is not recommended to enlarge photographs to fill the space. All the photographs of one morphological character should be the same magnification.

3. Clear photographs should be taken first and then software programs used to edit them. However, almost all the photographs in the plate should be in the same background colour and background colour should not be shiny and overshadow the fungal characters.

4. Morphological characters should not be excessively edited, especially colour and size. All editing software programs are facilitated for deleting, copying and pasting and those options are used to change the original photo. However, in scientific illustrations, natural characters and structures should not be changed too much as this may eliminate or hide important taxonomic characters (Fig. 11). 
5. Scale bars should be in the same width and all the labels should be in the same font size and style. Label and scale bars should be located at the same level of height of the photo.

6. The frame of all photographs should be of the same width.

7. Colony morphology should be compared when grown in the same culture media, same maturity and same incubation condition. Fungal colonies are morphologically different when grown in different culture media. Incubation conditions also affect the colony characters. Therefore, photographs of colonies should be taken when grown in the same medium for comparative purposes.

8. The measurements of morphological characters were obtained manually with a calibrated, low magnified microscope in early studies and the accuracy of those measurements is low. Currently, measurements are taken using the digital software and they are more accurate. There may be a variation in measurements of new collections and original Protologues of the same species. These variations cause errors on small measurements such as conidial size or spore length. Therefore, if original descriptions are incomplete, it is advisable to loan and examine the authentic specimens and obtain the measurements to compare the morphological characters with new collections.

9. The morphological structures examined from the specimens should always be compared with the morphological structures derived from the specimens, not from the cultures because the colour and size of the morphological structures derived from specimens may differ from those derived from their cultures. Therefore, it should be clearly stated whether morphological descriptions are obtained either from specimens or cultures.

10. The sample size is really important when calculating the mean of small values such as spores or conidia, asci, conidiogenous cells and conidiophores. However, there is no fixed sample size for morphological characters and the range of sample size varies from 10 to 50 in most studies. Based on the literature survey and statistical analyses in this study (not shown here), the minimum sample size is determined.

According to the statistical analyses here, there are no significant difference between the mean values $(\overline{\mathrm{x}})$, if the difference between minimum and maximum length is lower than $10 \mu \mathrm{m}$ (when $\mathrm{n}=$ 50 values). Ex: Ascospore length of Diatrype sp.; $6.4-11.5 \mu \mathrm{m}(\overline{\mathrm{x}}=8.7 \mu \mathrm{m}, \mathrm{n}=30)$

$$
\text { Measurement }_{\max }-\text { Measurement }_{\min } \leq 10 \mu \mathrm{m} \text {; then Sample size } \leq 50 \text {. }
$$

However, if the difference between the minimum and the maximum length of a character is higher than $10 \mu \mathrm{m}$ (between 50 values), then there is a significant difference between mean values $(\overline{\mathrm{x}})$. So that means the character shows a wide range of variation and more than 50 measurements should be considered to get an accurate value.If there are deviated measurements, then it is necessary to mention the minimum and maximum values of the range in brackets.

$$
\text { Measurement }_{\max }-\text { Measurement } t_{\min } \geq 10 \mu \mathrm{m} \text {; then Sample size } \geq 50 \text {. }
$$

Ex: Ascospore length of Neomicrosphaeropsis alhagi-pseudalhagi; (28.5)30.2-45.3(48.7) $\mu \mathrm{m}(\overline{\mathrm{x}}=$ $38.0 \mu \mathrm{m}, \mathrm{n}=50)$.

\section{Isolation of fungi}

Isolation means obtaining a genetically pure entity from a sexual or asexual morph of one or a mixture of fungi and pure cultures are elemental for any fungal experiment (Mueller et al. 2004a, Noman et al. 2018, Senanayake et al. 2020). Isolation is essential for many reasons. Cultures can provide important morphological characters useful for identification purposes as well as the starting material for DNA extraction useful for molecular studies. Ex-type cultures represent the taxon (Gams 2015). Cultures are one of the major sources to study and understand the biology of a species. Extracted DNA is used to obtain sequence data from a few genes, whole genomes, to transcriptomes under different conditions (Zeng et al. 2019). In addition, some taxa produce 
secondary metabolites that can be extracted from cultures (Strobel et al. 1996, Choi et al. 1999) and these are used as biocontrols, biopesticides, nematicides and enzymes (Chandler et al. 2011). However, contamination of cultures by other fungi can affect the final product on an industrial scale (Noman et al. 2018). Inbreeding, + and - hyphae of isolates are used in mating studies. These breeding cultures are used in mushroom production, biomedicine and also for fungal inoculum production in agriculture (Chandler et al. 2011).

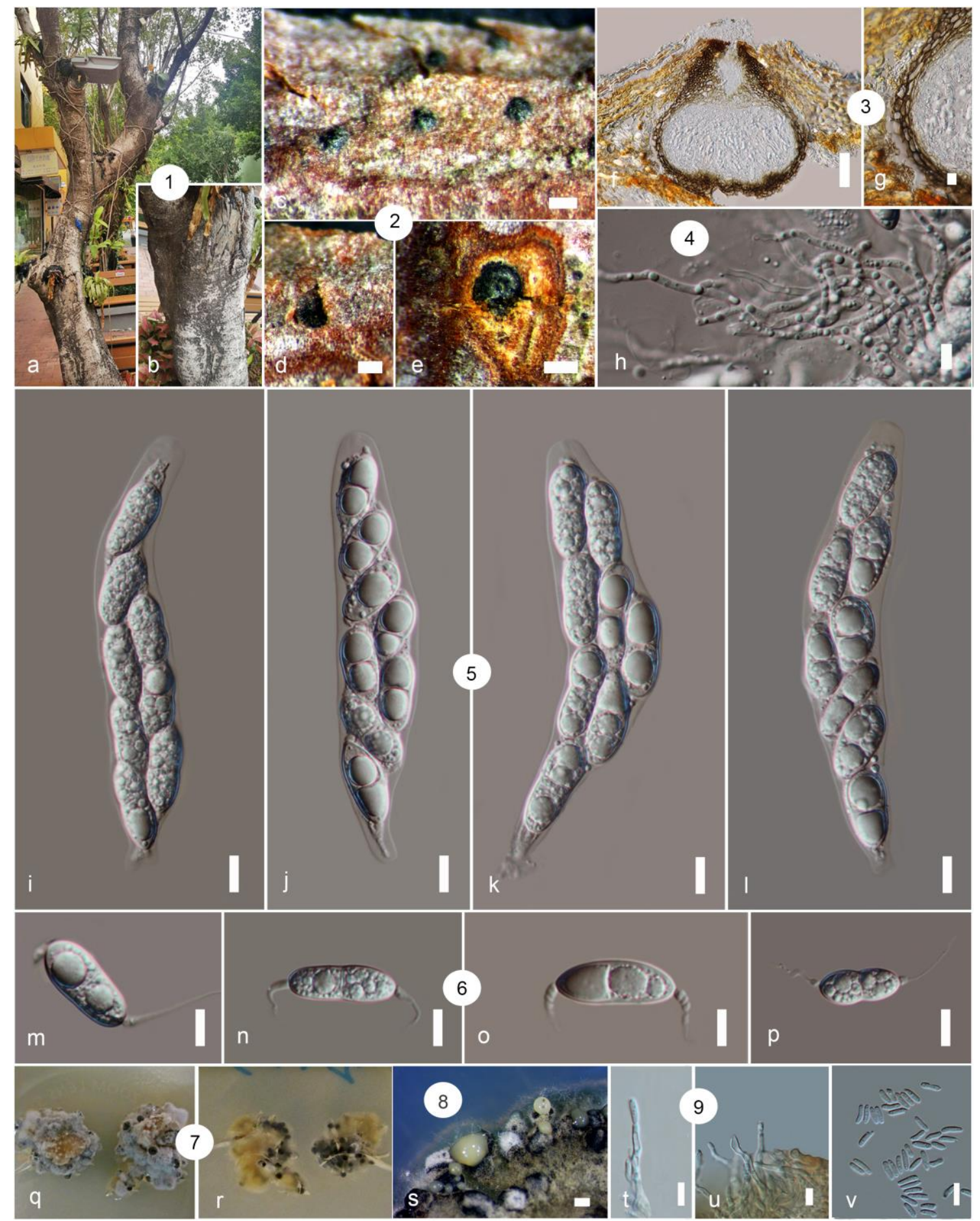

Figure 11 - A model illustration. 1 Collection details (e.g locality and host). 2 Appearance of fungus on substrate (disease symptoms, fruiting body arrangement and visual characters before dissecting). 3 Vertical or horizontal sectioning through the ostiole demonstrating periphyses, ostiole tissues and peridium characters (stromatic characters and chemical reactions of stromata if available). 4 Hamathecium tissues such as paraphyses, pseudoparaphyses, and cellular mass. 5 Asci 
arranged from immature to mature, natural to stained. 6 Ascospores arranged according to maturity and natural to stained; demonstrating sheath, appendages, globules, septa. 7 Pure culture to show colony characters. 8 Conidiation on cultures. 9 Conidiophores, conidiogenous cells and conidia.

Isolation of taxa from hosts or substrates and obtaining pure cultures has always been challenging (Goh 1999). Most mycologists consider that a pure culture should be obtained from a single germinated spore (Kirsop \& Doyle 1991, Goh 1999). Thus, single spore isolation is commonly used for obtaining pure cultures (Goh 1999). To establish relationships between sexual and asexual morphs, it is better to obtain isolates from single spores (Choi et al. 1999, Goh 1999, Chomnunti et al. 2011, Noman et al. 2018) in order to confirm that they are the same fungus.

Isolation methods should be simple, inexpensive and effective (Choi et al. 1999). Some species produce numerous fruiting bodies, others produce only a few (Goh 1999) or sometimes fruiting bodies are not fully matured to enable identification. Collection of mature fruiting bodies or an adequate number of spores is needed (Goh 1999). Conidia of contaminants on the specimens can be transferred along with conidia or spores (Bernadovičová \& Ivanová 2011). If the species is slow growing, then bacterial or fungal contaminants may overgrow them and may result in identifying the wrong species (Smith 1969, Goh 1999). Some bacteria can inhibit growth even if the culture is prepared in antibiotic incorporated media (Smith 1969, Goh 1999).

Since not all spores germinate in artificial media, the development of other techniques has been necessary (Choi et al. 1999). In addition to single spore isolation, multiple spores or spores attached to the fruit body walls are isolated to obtain cultures. Some taxa need specific culture media to maintain their metabolic activities (Smith \& Onions 1994, Leyronas et al. 2012). Fungal mycelia obtained in pure culture are also essential for physiological and chemical profiling of the strain of interest (Noman et al. 2018).

If the isolated taxon is contaminated with other fungi or bacteria, then purification is required to obtain a pure culture. Techniques including hyphae tip culture (Fig. 12) or single colony subculture into new media (Choi et al. 1999, Noman et al. 2018). Hyphae tipping can be used when the growth rate of the taxon is greater than the contaminant (Choi et al. 1999, Goh 1999, Rangaswami \& Mahadevan 2006). For some taxa that cannot grow in artificial media, it is recommended to extract DNA directly from fruiting bodies as prescribed by Zeng et al. (2018). Goh (1999) proposed the hand-made glass needle technique to purify the spores before isolation. Herein, the spores are rolled by the dragging movement on the agar surface and contaminants on the surface of the spore are removed by repeating rolling.

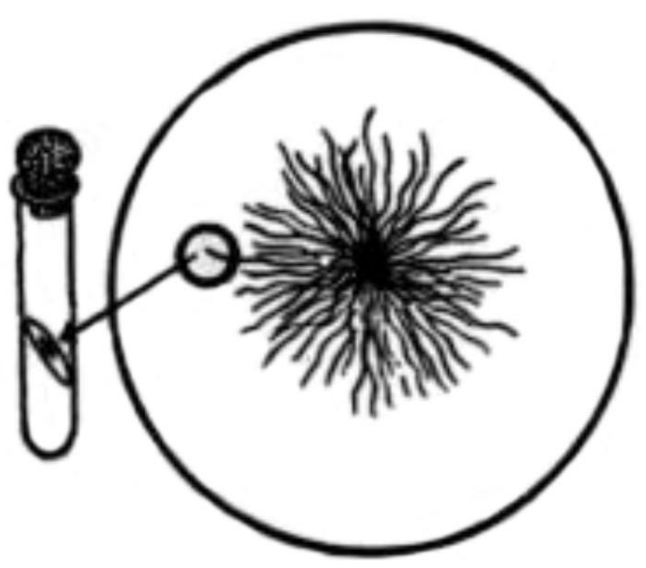

(a)

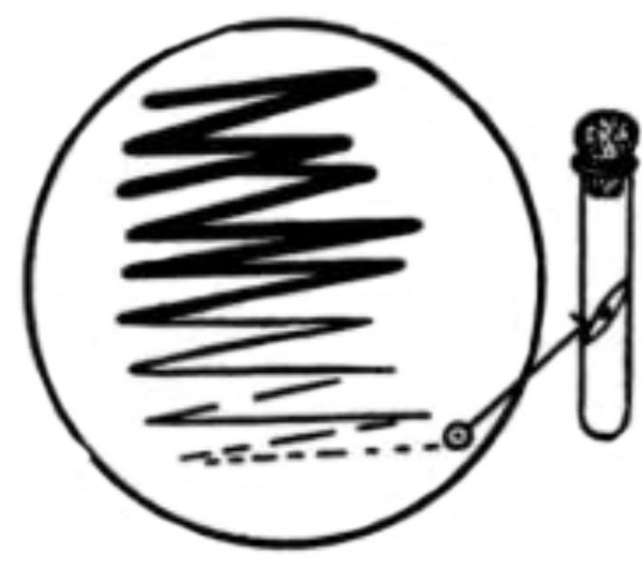

(b)

Figure 12 - Culture purification methods (Redrawn from Rangaswami \& Mahadevan 2006). a Single hyphal tip culture method. b Single colony subculture method. 


\section{Culture media}

Culture media generally contains a high percentage of carbohydrates and nitrogen (at a $\mathrm{pH}$ range of 5-6), which facilitates growth. Incubation normally ranges from $15-37^{\circ} \mathrm{C}$ (Basu et al. 2015). Natural and synthetic media are the two general types of media. Natural media are made from herbaceous or woody stems, seeds, leaves, cornmeal, wheat germ, oatmeal and starchy substrates (Collins et al. 2005). The composition of natural media is undetermined and corn meal agar, potato dextrose agar, V8 juice agar, dung agar are just a few examples of natural media (Fig. 13). The composition of ingredients in synthetic media such as carbohydrates, nitrogen and vitamins is quantified (Basu et al. 2015).

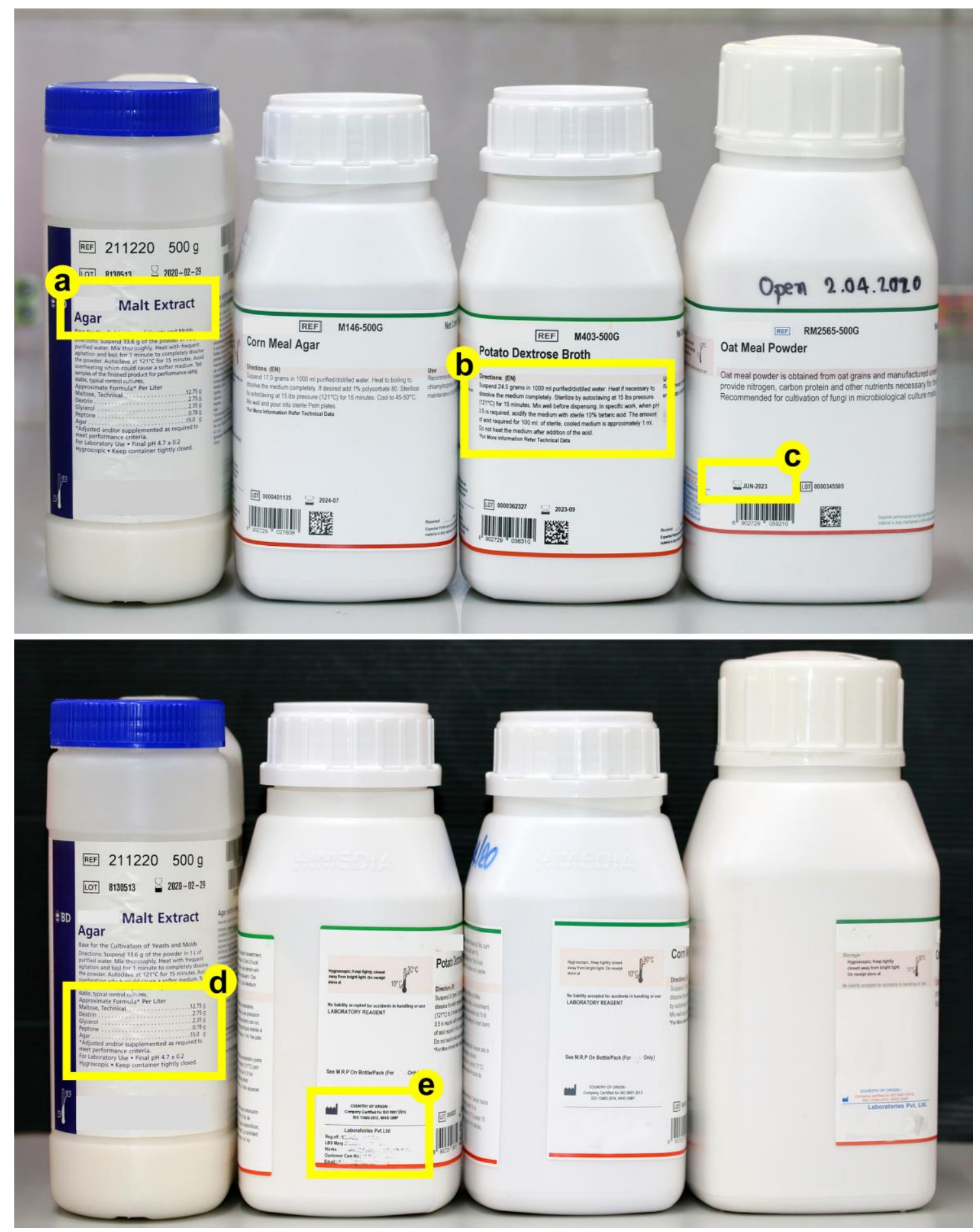

Figure 13 - Important information on culture media labels. a Name of the media. b Concentration to prepare. c Production and expiry date. d Composition. e Producer and distributor. 
Different media are used for different fungal groups and the selection of media depends on the group being studied (Basu et al. 2015). Generally, spore suspensions are used for isolation and may contain different spores. Therefore, spores are carefully examined under a stereomicroscope and germinating spores are transferred to new media just after the formation of germ tubes (Basu et al. 2015). Nutrients deficient medium such as water agar is suitable for this. If nutrient rich media are used for isolation, then contaminant spores may germinate faster than targeted species ( $\mathrm{Su}$ et al. 2012).

Generally, nutrient-rich media with a low percentage of agar are used for subculturing. This releases nutrients easily and retains moisture in the media (Braun et al. 2011). Cornmeal agar, czapek yeast autolysate agar, malt dextrose agar, malt extract agar, potato carrot agar, potato dextrose agar, potato sucrose agar, V8 vegetable juice agar, yeast extract-phosphate medium, are the commonly used media which facilitate colony formation (Su et al. 2012).

Fungal sporulation usually occurs under unfavorable growth conditions (Dahlberg \& Etten 1982). Many taxa need nutritional elements such as carbon, nitrogen and microelements for their growth (Timnick et al. 1951), while some taxa have specific carbon and nitrogen requirements (Engelkes et al. 1997, Gao et al. 2007). However, most of the commonly used media provide favorable growth conditions for most taxa (Su et al. 2012) but are frequently less successful in inducing sporulation (Guo \& Michaelides 1998, Li et al. 2007). Starvation or nutritional depletion often stimulates sporulation and therefore, artificial media with low nutrient content, such as water agar, half- or 1/4-strength PDA and synthetic nutrient-poor agar induce sporulation (Nirenberg 1976, Masangkay et al. 2000, Wulandari et al. 2009, Braun et al. 2011).

Mycelia are grown on a thin layer of media to store the cultures. Metabolic activities of the mycelia are deactivated by storage in the low temperature or in oxygen-free oil. Therefore, general media such as cornmeal agar, malt dextrose agar, malt extract agar, potato dextrose agar, potato sucrose agar and V8 vegetable juice agar are used for this. Conventional media are often unsuccessful for culturing extremophiles under high or low temperature, pressure and $\mathrm{pH}$ conditions (Tsudome et al. 2009). Thermophilic taxa cannot be cultured on cellulose and agar plates, as those media are liquefied above $70^{\circ} \mathrm{C}$ for extended periods of time (Tsudome et al. 2009). Porous solid plates made of nanofibrous cellulose have been developed and these plates can withstand temperatures of up to $260^{\circ} \mathrm{C}$ at $25 \mathrm{MPa}$ (Tsudome et al. 2009). Porous solid plates support the growth of a wide range of extremophiles including acidophiles, alkaliphiles, thermophiles, acidothermophiles and alkalithermophiles under extreme physiochemical conditions (Basu et al. 2015).

\section{Preparation of media, apparatus and aseptic conditions}

A sterile work environment is essential for the isolation of fungi. Appropriate preparation and sterilization of both media and apparatus are very important for the successful isolation of targeted species and avoid contaminants (Basu et al. 2015). Suitable solid media is prepared using distilled water mixed with the appropriate amount of selected powdered agar. Powdered agar is now commercially available or can be prepared according to the manufacturer's protocols (Crous et al. $2009 \mathrm{~b}$ ). The powdered media mixed with the water is then heated in a microwave oven until all the agar dissolves and a clear solution is obtained (Gao et al. 2007). The medium is then sterilized in appropriate media bottles using an autoclave set at $121^{\circ} \mathrm{C}$ for 20 minutes or as specified otherwise. The media is allowed to cool and antibiotics are added if necessary, to suppress the growth of bacterial contaminants. Medium is then poured into Petri-dishes in a laminar flow cabinet and the latter should be disinfected and cleaned before hand (Fig. 14).

All tools used for the isolation are autoclaved or flame-sterilized. Plastic Petri-dishes are sterile and do not require further treatment. Glass Petri-dishes are sterilized in a hot air oven at $160^{\circ} \mathrm{C}$ for 4 hours (Longcore et al. 1995). Since pouring of media should be conducted in a sterile environment, the UV-lamp in the laminar flow cabinet should be switched on for at least 20 minutes to sterilize the interior of the hood (Crous et al. 2009b). Subsequently, the floor of the laminar flow cabinet is cleaned with $70 \%$ alcohol (Longcore et al. 1995). Pouring of a medium into 
plates, culture, subculture or other inoculations should be carried out near the alcohol lamp to keep contamination at minimum.
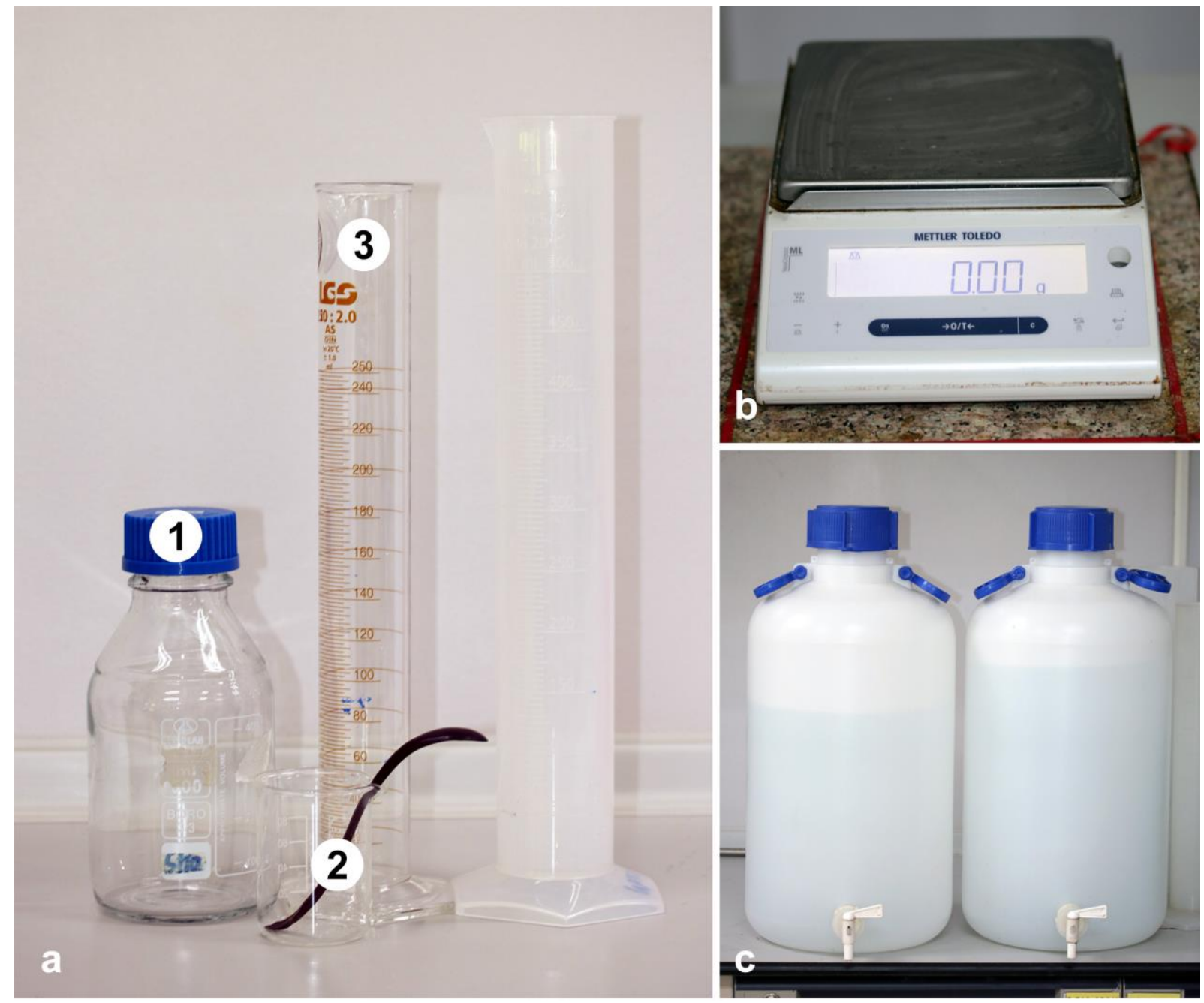

Figure 14 - Materials and tools needed for media preparation. a Materials used to prepare media (1) Media bottle, (2) Spatula in a beaker, (3) Graduated cylinder. b Precision balance. c Distilled water in polyethylene containers.

\section{Preparation of specimens for isolation}

\section{Surface sterilization of specimen}

Bacteria, other fungi, insect eggs, soil and other debris are often associated with specimens, and all may cause problems during isolation (Ataides et al. 2018). Therefore, it is necessary to clean the specimens as much as possible (Jan et al. 2013). Surface sterilization involves treating the material with a strong oxidant (hydrogen peroxide, Ozone, Chloride) or disinfectant (70\% alcohol, UV radiation) for a short period carried out carefully. Surface sterilization is carefully applied in the case of superficial species and hyphomycetes. Procedures outlined by Stone et al. (2004) can be followed for isolating endophytes.

Surface sterilization is important to isolate endophytic fungi. Plant samples are rinsed using running tap water for around 15 minutes and then washed with surfactants for one minute. Surfactants commonly used in endophytic studies such as Tween 20, Tween 80, or Triton X-100, N-Dodecyl-Beta-D-Maltoside (DDM) and Digitonin (Gan et al. 2017, Mucciarelli et al. 2002, Adnan et al. 2018). The plant tissues are washed repeatedly with sterile water after each treatment (Bamisile et al. 2019, Xia et al. 2019). Samples are then washed with sterilizing agents such as 15\% Sodium hypochlorite for 2-10 minutes (Gardner \& Szabo 1982, Weber et al. 1999), 70-95\% 
ethanol for 0.5-4 minutes (Dong et al. 1994), Hydrogen peroxide (McInroy \& Kloepper 1995, Dastogeer et al. 2018) and Mercuric chloride 0.05-0.2\% for 2-5 minutes (Ahmed et al. 2012, Zhong et al. 2017, Rather et al. 2018). Rose Bengal or aniline blue is used to stain endophytic fungi. Different treatment durations and combinations can be used depending on the study or plant tissue of interest.

\section{Isolation methods for basal fungi}

Basal fungi produce both motile and non-motile sporangiospores (Longcore et al. 1995) and among them, members of Aphelidiomycota, Blastocladiomycota, Chytridiomycota, Monoblepharomycota, Neocallimastigomycota, Olpidiomycota and Rozellomycota produce motile spores (Powell 2017, Hurdeal et al. 2020). These organisms require different isolation techniques depending on the purpose of isolation, habitats, mode of life, sample types, propagule sizes and fungal density in the sample (Longcore et al. 1995). For example, obligate biotrophs require a host culture, while baiting is required to isolate the zoospores of saprobes (Powell 2017). Different methods are reviewed here that have been used by various researchers for the isolation of basal fungi. The diversity of fungi is affected by environmental factors, nutrient composition of culture media and isolation methods (Longcore et al. 1995).

Specific culture media are needed to isolate basal fungi because of their particular chemical composition and nutritional modes. Some media are suitable for a particular basal group, while others may be unsatisfactory (Benny 2008). Members of Chytridiomycota are saprobes or parasites in or on organisms (Ogawa et al. 2001, Hurdeal et al. 2020). Niches of Chytrids are pollen grains, insect exoskeletons, protists and small invertebrates, amphibian skin, other fungi, pieces of plants, fruits, and water-logged twigs (Boyle et al. 2003). These substrates are therefore used as baits to trap chytrids. The commonly used media for isolation and growth of Chytridiomycota are peptonized milk-tryptone glucose (PmTG), yeast phosphate soluble starch (YpSs), Archimycete agar (ARCH), and cornmeal agar (CMA) (Emerson 1958, Barr 1986, Longcore et al. 1995, 1999, Crous et al. 2009a).

Most species of Mucoromycota and Kickxellomycota can be isolated in pure culture on laboratory culture media, such as malt extract agar, malt-yeast agar, oatmeal agar, potato dextrose agar and Sabouraud dextrose agar (Bärtschi et al. 1991, Strauss et al. 2000, Benny 2008, Chuang et al. 2017). Neocallimastigomycota colonizes the rumen of herbivores. They are generally grown in small batch cultures without agitation on soluble or insoluble carbon sources with $100 \% \mathrm{C}$ or $70 \%$ C: $30 \% \mathrm{~N}_{2}$. The culture media for rumen anaerobic fungi are complex, non-defined media $(\mathrm{pH} 6.5-$ 6.8) and contain up to $15 \%$ (v/v) clarified rumen fluid (Davies et al. 1993).

\section{Baiting of motile sporangiospores of basal fungi}

Baits are commonly used to attract zoospores of basal fungi, especially members of Chytridiomycota and Blastocladiomycota from soil and water samples. Commonly used baits are chitin, purified shrimp exoskeleton, pollen grains, snake skin, and cellulosic substrates such as cellophane, onion skin, lens paper (Barr 1987). It is advisable that baits are sterilized under UV light before use (Jerônimo et al. 2019). Baiting techniques result in pure cultures from motile sporangiospores of basal fungi and several species of chytrids such as Boothiomyces macroporosum, Chytriomyces hyalinus and Rhizoclosmatium globosum have successfully been isolated using this technique from soil and water (Karling 1977, Kinsey et al. 1999, MozleyStandridge et al. 2009).

Materials required - Petri-dish, pipette with bulbs, baits, sterile distilled water, compound microscope, sterile needle, parafilm, antibacterial added culture media, stereo-microscope, micropipette, funnel, filter papers (same size with Petri-dish), glass spreader.

Methodology - If the samples are soil or any organic material, then 1-2 $\mathrm{g}$ of the sample is added to a sterilized Petri-dish along with the selected bait. Additional amounts may cause excessive bacterial growth. Sterile distilled water is then added to the Petri-dish (Karling 1977). The plates are then incubated at $25^{\circ} \mathrm{C}$. Periodically, the baits are observed to determine the 
presence of sporangia using a compound or dissecting microscope. Once sporangia can be seen on baits, the zoospores can be isolated to obtain an axenic culture (Letcher et al. 2014).

If the samples are water, then water samples are passed through a sterilized filter paper placed in a funnel and then, filter papers are incubated directly on the media. It may be necessary to concentrate samples by centrifugation (Mozley-Standridge et al. 2009). Sample enrichment is carried out by adding the sample to sterile nutrient broth in shake flasks at $12^{\circ} \mathrm{C}$ for 72 hours or $22^{\circ} \mathrm{C}$ for 48 hours. $1 \mathrm{~mL}$ of sub-samples is then plated on the media and plates are examined occasionally for the appearance of fungal colonies (Jerônimo et al. 2019).

\section{Cavity slide method for isolating motile sporangiospores of basal fungi}

This method is used to isolate mature sporangia from a natural substrate or bait before it is covered by undesirable microorganisms. The concept is to allow the release of spores in a determined place for isolation (Mozley-Standridge et al. 2009).

Materials required - fine isolation needles, forceps, micro-scissors, microscopic slides, slide coverslips, cavity slides, Petri-dish damp chamber, alcohol lamps, marker pens, antibacterial added agar plates, parafilm, sterile distilled water, glass spreader, stereomicroscope, compound microscope.

Methodology - A part of the bait bearing the most sporangia is transferred to the cavity of a cavity slide with few drops of sterile water. Another sterilized, inverted cavity slide is placed on top of the first cavity slide with the specimen. This avoids the evaporation of water. The cavity slides are placed in a damp chamber on a triangle of bent glass in a Petri-dish. Few drops of water are placed in the Petri-dish to maintain moisture. A compound microscope is used to observe the cavity slide for the release of zoospores from the mature sporangia. Once zoospores are released and swimming in the water, they are transferred to the $50 \mu \mathrm{L}$ of sterile water. Several drops of this spore suspension are pipetted onto the agar plates containing antibiotics and marked where the suspension drop using a marker pen (Mozley-Standridge et al. 2009).

\section{Sporangia isolation method}

This is the method to isolate zoospores, by which the sporangium/sporangia are directly isolated before they release the zoospores (Powell 2017, Jerônimo et al. 2019).

Materials required - agar plates, sterile needles, parafilm, sterile pipettes, microscope.

Methodology - A single sporangium is picked using a pipette or needles from the baits and is transferred directly onto any agar media. A sterile loop needle is used to drag the sporangium through the agar to remove any contamination and the sporangium is then transferred to a fresh agar plate and sealed with parafilm. When the sporangium releases the zoospores, those can be further isolated by transferring them onto fresh media.

\section{Co-culture method}

This method is used to isolate the obligate biotrophic basal fungi Aphelidiomycota, Rozellomycota and some members of Chytridiomycota (Jerônimo et al. 2019). Co-culture or mixed cultures involve the growth of two different microorganisms together. This culturing technique allows synergistic or antagonistic interactions and for basal fungi, co-culture refers to the growth of the fungi with the host organisms (Boyle et al. 2003, Carter \& Shieh 2015).

Materials required - host culture, micropipette, filters, media, gauze, pipettes, microscopic slides, compound microscope

Methodology - Water samples are mounted on a microscope slide and examined using a compound microscope for any symptoms of infection of algae, diatoms, dinoflagellates and other planktonic microorganisms. If the above microorganisms are available, then microorganisms with the fungal sporangia are transferred to either solid or liquid media using micropipettes. Usually, F/2 liquid medium or mineral medium are used. If the host culture is available, then the host is cultivated in the media first. The isolated fungi are then transferred to the host organism using pipettes. The host is cultivated with the fungi until the maximum infection is observed (Garvetto et 
al. 2019). The fungus is then harvested and obtained in cultures (Karpov et al. 2017). Alternatively, water samples can be filtered through gauze and the filtrate can then be inoculated with host culture in nutrient media such as F/2 liquid medium and N1 medium (Karpov et al. 2016).

\section{Hungate roll tube/ Serum roll bottle technique for isolation of anaerobic fungi}

This technique was devised for the cultivation of anaerobic microorganisms (Neocallimastigomycota) and originally described by Hungate (1969). Several variations exist for the roll tube method, whereby the serum roll bottle technique is one of the most widely used (Miller $\&$ Wolin 1974). The technique is widely used for acquiring pure cultures of anaerobic fungi. These fungi inhabit the guts of ruminants. In the roll tube method, anaerobic agar medium is distributed on the internal surface of a test-tube under anaerobic conditions (Hungate 1969).

Materials required - Glass serum tubes, glass test tubes, culture media, Balch stopper, aluminum seals for them, micropipette and tips, loops and needles, anaerobic chamber, heater, gassing probe, antibacterial added media plates, anoxic gas mixture, incubator, antibiotics and microscope.

Methodology - Immediately collected fresh samples, such as fecal matter or rumen digesta from herbivores are homogenized, and dilutions are made from $10^{-1}$ to $10^{-6}$ using an anaerobic diluent or rumen-fluid cellobiose (RFC) media (McSweeney et al. 2005, Calkins et al. 2016).

Culture media are heated to boiling point while eliminating dissolved oxygen by a gassing probe with high-grade $\mathrm{CO}_{2}, \mathrm{~N}_{2}$, or mixtures of $80 \% \mathrm{~N}_{2}$ and $20 \% \mathrm{CO}_{2}\left(\mathrm{~N}_{2}: \mathrm{CO}_{2}\right)$ or $80 \% \mathrm{H}_{2}$ and $20 \% \mathrm{CO}_{2}\left(\mathrm{H}_{2}: \mathrm{CO}_{2}\right)$. An anoxic gas mixture filled plastic syringe $(10 \mathrm{~mL})$ is used to flush the anoxic gas into the media and take out culture media under an $\mathrm{O}_{2}$ free condition (Wolfe 2011). During the whole procedure, anoxic gas should pass through the gassing probe to maintain anaerobic conditions. Then $10 \mathrm{~mL}$ of culture media is transferred to the Hungate sealing tube filled with anoxic gas in an anaerobic chamber. This tube is sealed with a Balch stopper and crimped an aluminum seal (Olson 1992, Metcalf et al. 1998).

After sealing the tubes, they are opened in an anaerobic chamber when required. The inoculum is added to the glass serum tube containing culture medium supplemented with antibiotics and tube is sealed with a Balch stopper and crimped with an aluminum seal. Then tubes are shaken and rolled on the bench surface to slowly solidify the media and this increases the surface area for fungal growth. The roll tubes are incubated at $39^{\circ} \mathrm{C}$ for $2-3$ weeks, whereby fungal growth is monitored regularly using a microscope. When distinct colonies form, they are picked aseptically under anaerobic conditions and transferred to a fresh liquid culture medium. Subsequently, the roll tube method is repeated several times until a pure culture is obtained (Hanafy et al. 2018, Joshi et al. 2018).

For isolation of anaerobic fungi from saliva samples, roll tubes containing $2 \mathrm{~mL}$ molten defined medium B with $1.8 \%(\mathrm{w} / \mathrm{v})$ agar and either $25 \mathrm{mM}$-glucose or $1 \%(\mathrm{w} / \mathrm{v})$ cellulose agar supplemented with antibiotics are prepared and then inoculated with $0.1 \mathrm{~mL}$ saliva. The tubes are incubated at $39^{\circ} \mathrm{C}$ for 3 days and examined for colonies using a stereo-microscope (Lowe et al. 1987).

For the isolation of anaerobic fungi from the rumen, samples of rumen liquor are flushed with $\mathrm{O}_{2}$-free $\mathrm{CO}_{2}$ for a minute and diluted $\left(10^{-1}\right.$ dilution) in an anaerobic diluent containing $2 \%(\mathrm{v} / \mathrm{v})$ antibiotic stock solution of Benzylpenicillin, Streptomycin sulfate, and Chloramphenicol (each 5 $\mathrm{mg} / \mathrm{mL}$ ) (McSweeney et al. 2005). Roll tubes are then prepared with antibiotics by injecting $0.5 \mathrm{~mL}$ of a $10^{-1}$ dilution into $50 \mathrm{~mL}$ serum bottles containing $5 \mathrm{~mL}$ cellobiose agar medium (Miller \& Wolin 1974). Joshi et al. (2018) proposed that, samples of rumen digesta are pooled and homogenized for 10 minutes under a gas phase of $\mathrm{CO}_{2}$ before preparing dilutions (up to $10^{-4}$ ) in the anaerobic diluents.

For the isolation of anaerobic fungi from soil, a serial dilution is performed on $1 \mathrm{~g}$ of soil samples using $10 \mathrm{~mL}$ of $0.03 \%$ cysteine hydrochloride solution $(\mathrm{pH} 7.0)$ and the $10^{-1}$ to $10^{-3}$ dilution series are done. A diluted sample $\left(1 \mathrm{~mL}\right.$ of $\left.10^{-3}\right)$ is transferred to a solidified agar medium on roll tubes and then tubes are sealed under an oxygen free condition. Tubes are incubated at $25^{\circ} \mathrm{C}$ 
in a vertical position (Hungate 1969, Tonouchi 2009). Fungal colonies are transferred to the same anaerobic fresh agar slant and incubated at $25^{\circ} \mathrm{C}$, then transferred to PDA plates and incubated at $25^{\circ} \mathrm{C}$ under anaerobic conditions.

\section{Dilution plating method for non-motile sporangiospores producing basal fungi}

The dilution plating method can be used for isolation of basal fungal groups, such as Mucoromycota and Mortierellomycota from the soil and fecal samples of animals. The dilution method entails a serial dilution of samples. Apophysomyces thailandensis, a new species of basal fungus from soil was isolated using the dilution plating method (Khuna et al. 2019). Mostly, selective media are used for the isolation of mucoralean fungi (Strauss et al. 2000). Bärtschi et al. (1991) recommended malt yeast agar supplemented with an antibacterial for the isolation of Mucor species. Tansey (1984) isolated thermophilic and thermotolerant mucoralean fungi from numerous materials using selective media and incubated samples at $45^{\circ} \mathrm{C}$ (Fig. 15).

Materials required - culture media plates, glass spreader, parafilm, a sieve set, electric balance, measuring cylinders, sterile distilled water, test tubes, flasks and lids, shaker, incubator.

Methodology - Soil samples are sieved through a $2 \mathrm{~mm}$ sieve. Then a series of dilutions is performed using $1 \mathrm{~g}$ of soil is added to $10 \mathrm{~mL}$ of distilled sterile water or $5 \% \mathrm{NaCl}$. The mixture is then shaken for around one to two hours with 100-200 rpm. Then, $0.1 \mathrm{~mL}$ of this suspension is plated on an agar plate and is spread using a sterile glass spreader. Subsequently, the agar plate is sealed and incubated. The plates are periodically observed and when fungal colonies are seen, they are transferred to a fresh agar plate (De Souza et al. 2018, Nguyen et al. 2016, 2018).

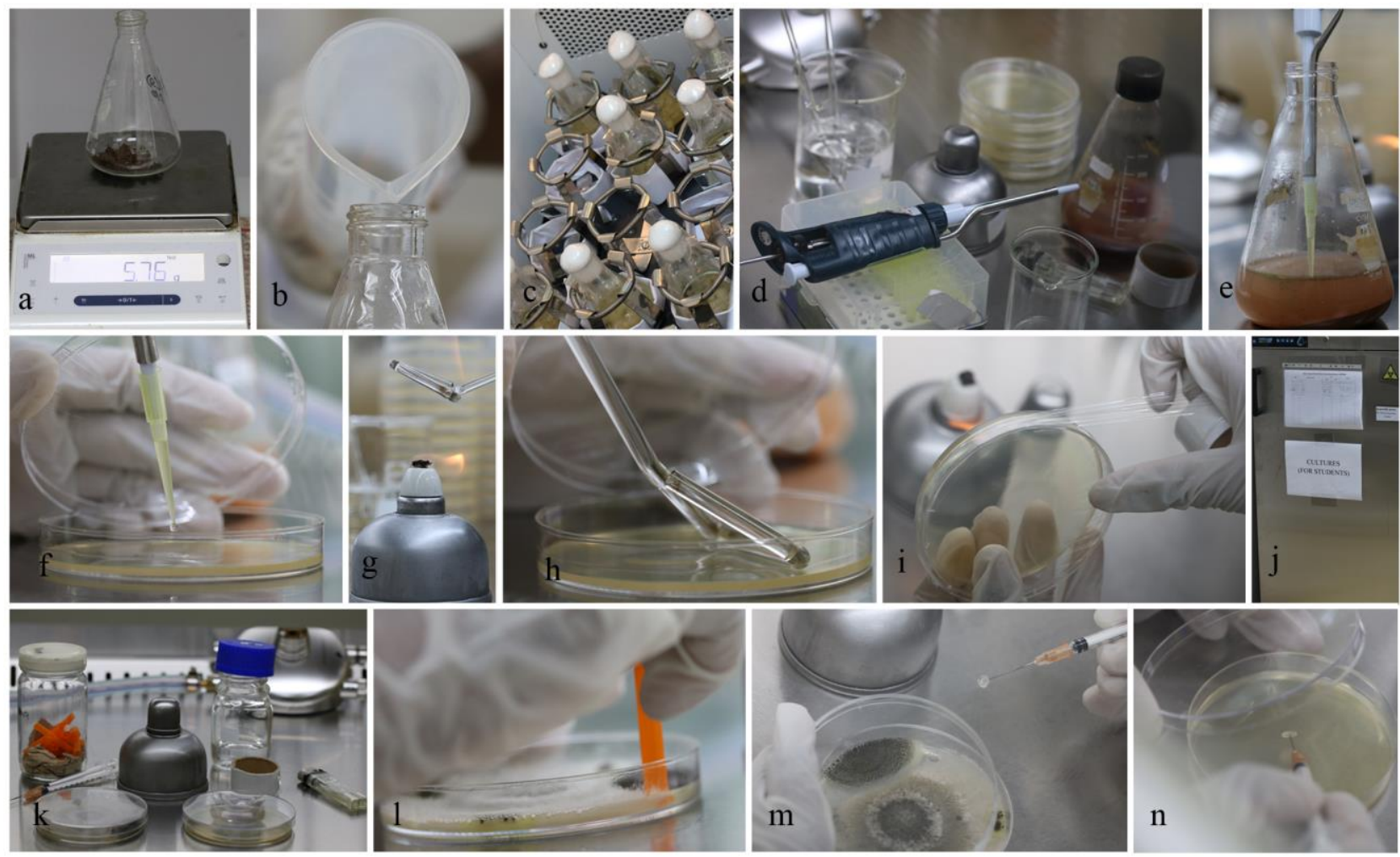

Figure 15 - Dilution plating method. a Weight the soil. b Dilute with sterile distilled water. c Put the flasks containing the mixture in an incubator shaker at 180 to $200 \mathrm{rpm}$. d Materials needed to inoculate an agar plate with the soil suspension. e-f Transfer the suspension to a fresh agar plate using a sterile tip. g Flame sterile a glass spreader. h Spread the soil suspension using the sterile and cooled spreader. $\mathrm{i}-\mathrm{j}$ seal the plate and incubate the agar plates. $\mathrm{k}$ Materials needed to transfer colonies from inoculated plate to fresh media. 1 Use a sterile straw to cut the mycelia tip and the agar. m-n Use a flame-sterilized needle to pick up and transfer the mycelial plug to a new agar plate. 


\section{Direct inoculation method}

Direct plating is the best method to isolate mucoralean fungi from dung (Krug 2004, Benny 2008). Species of Absidia, Circinella, Cunninghamella, Lictheimia, Mucor, Pilobolus, Rhizopus, Syncephalastrum and Thamnostylum are often isolated using this method from dung and soil (Benny 2008, De Souza et al. 2016).

Materials required - Agar plates, sterile forceps, parafilm.

Methodology - Samples such as water, soil and fecal content are directly placed on agar media containing antibiotics. The agar plates are then incubated at $25-28^{\circ} \mathrm{C}$. Once fungal growth is seen, colonies are transferred to fresh agar media to further isolate the fungi (De Lima et al. 2018).

Alternatively, the bottom of the Petri-dishes is lined with one or two layers of filter paper or paper towel and dung samples from small animals such as rats and mice are placed on the top of the filter papers (Benny 2008). Then, the plates are incubated in the moist chambers for 3-10 days. Mucoralean fungi can be observed under a stereomicroscope (Fig. 16).

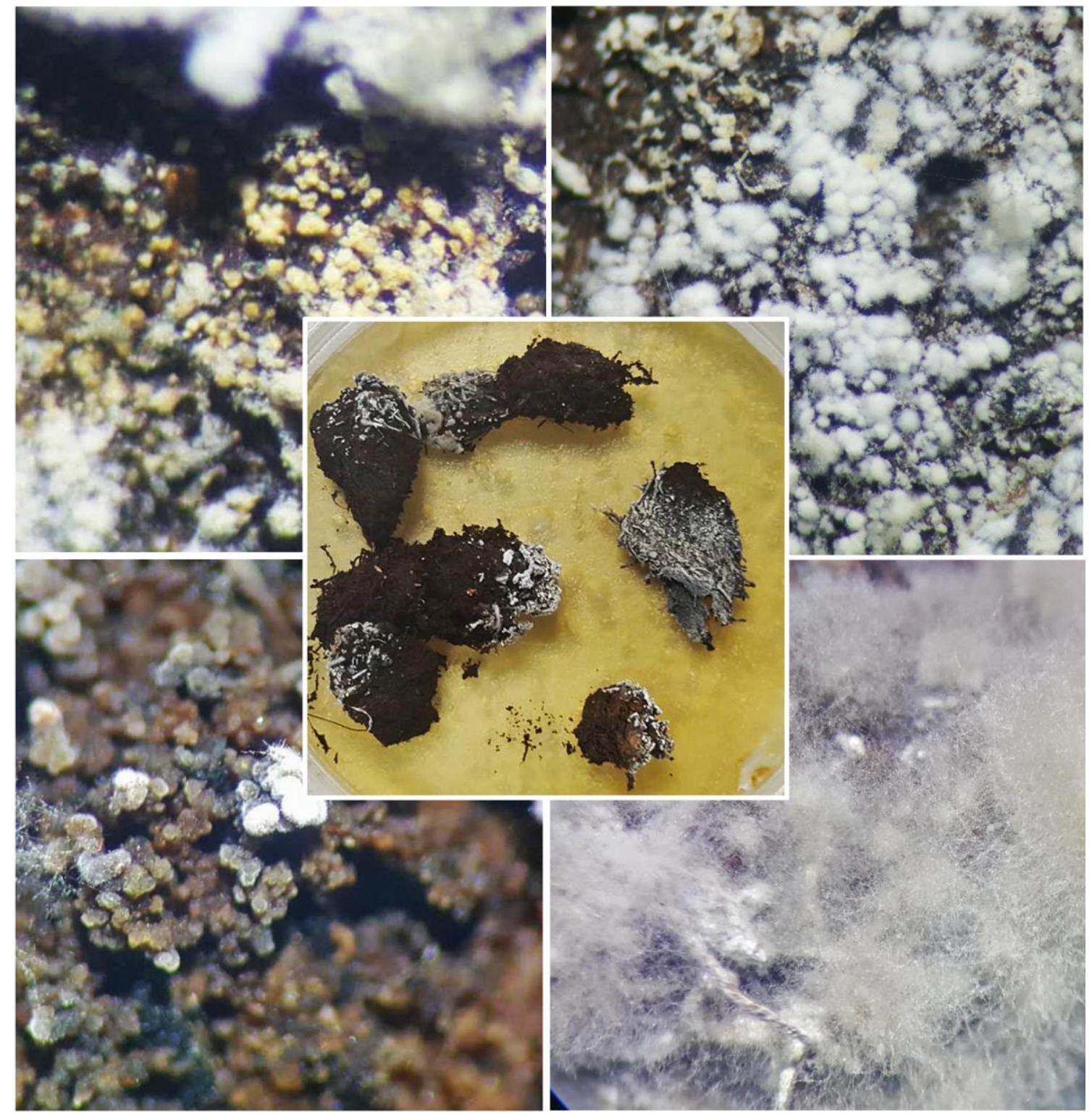

Figure 16 - Fungi isolated from direct inoculation method.

\section{Wet sieving method}

The wet sieving method is generally used to isolate large spores producing soil fungi and hyphal pieces of mycorrhizal fungi (Thorn et al. 1996, Hoysted et al. 2018). Arbuscular 
mycorrhizae fungi generally produce larger spores than other fungi ranging from $50-500 \mu \mathrm{m}$ diam. (Blauda et al. 2016).

Materials required - sterilized needles, filter papers, ethanol lamp, distilled water, disinfectant, marker pen, centrifuge tubes, Petri-dishes containing about $10 \mathrm{~mL}$ of PDA, Standardized analytical sieves for analytical sieve shakers of the series AS 200, 50\% sucrose solution, compound microscope.

Methodology - Spores are directly extracted from material in soil samples. Soil samples mixed with water or $50 \%$ sucrose solution are stirred. This suspension is poured onto the top sieve of the wet sieving stack. The sieve shaker is turned on for 5-10 minutes depending on the size of the sample. While the sample is being sieved, water is added continuously to wash the samples. If the liquid from the outlet is clear, then the samples retained on each sieve are collected and they are dried using an oven. After extraction, spores are carefully selected using a compound microscope. Spores are further cleaned by ultrasonication for 15 seconds and are rinsed in sterilized distilled water. Clean and matured spores are selected from the material observing by a compound microscope and transferred to $1.5 \mathrm{~mL}$ microcentrifuge tubes containing sucrose using a sterilized needle. Tubes are stored at $-80^{\circ} \mathrm{C}$ until required and spores are used for culturing in the agar media when necessary.

\section{Isolating techniques for hyphomycetes}

Isolation of hyphomycetes can be difficult due to their exposure to the external environment (Subramanian 1983). These fungi can be easily contaminated by other fungi and bacteria. Preventing the growth of non-targeted fungi is the most important strategy to obtain a pure culture of targeted hyphomycetes (Ellis 1971). However, different methods are used for isolating hyphomycetes, some of which are described below.

\section{Direct streaking method}

This is a method used to obtain pure cultures from hyphomycetes or fungi that have a large number of spores. Here, spores are directly streaked on culture media under aseptic conditions without making a spore suspension. This method uses only several tools and saves time (Andrews \& Pitt 1986).

Material required - Culture media, needle, razor blades, $70 \%$ alcohol, tissues, sterile water, parafilm, stereomicroscope

Methodology - Fungal specimen surface is sterilized using a tissue paper moistened with $70 \%$ alcohol without disturbing conidial attachment. Then, a needle tip is dipped in sterilized water and slowly moved across the fungus surface and conidia stick to the needle surface. A few conidia can be placed on a sterilized, moisten needle. Spores or conidia picked by the needle are streaked directly on freshly prepared culture media under aseptic conditions. Plates are then incubated to facilitate conidia germination and germinated conidia are transferred to the fresh media.

\section{Glass needle technique}

This method was introduced by Goh (1999) to isolate various taxonomic groups of fungi including hyphomycetes using single conidium isolation. The method has been successfully used in the isolation of dematiaceous hyphomycetes, which have large spores (Goh 1999).

Material required - 2 Pasteur pipettes, alcohol burner, beaker, folded paper towel, culture media.

Methodology - The glass needle preparation is the initial step used in this method. The entire narrow front portion of the two glass Pasteur pipettes are broken. Then broken pipettes are held horizontally with the conical parts facing each other and the broken tips of the Pasteur pipettes are heated in the flame of an alcohol-burner to join them while melting and then pulling the glass pipettes apart. This should be done inside an enclosed compartment, such as the laminar flow (with the airflow turned off), fume cupboard or any other enclosed cabinets. It is essential to keep the 
flame of the burner vertical during the joining and pulling of the pipettes. If the flame is not vertical, then the glass would become fragile and the needles would not be flexible (Goh 1999).

Once an ideal glass needle has been made, it can be used for single spore isolations. A small cavity is made by a sterile scalpel on a fresh $3 \%$ water agar plate under a dissecting microscope in an aseptic condition. Then, the natural substratum or a minute sample of the mycelium with spores is placed onto the cavity made on the water agar. Then a small part of the sample is dragged on the agar sample and while dragging spores are separating from each other. The separated spores are transferred to fresh culture media for spore germination. Germinated spores are transferred to fresh plates for colony formation (Goh 1999).

\section{Soil plate method for isolation of soil hyphomycetes}

This method can be used for isolation of soil hyphomycetes and it is necessary to follow manufacturer's protocol to prepare media for optimum fungal growth and sporulation (Barron 1972).

Material required - Petri-dishes, Sterilized water, Czapek-Dox $+0.5 \%$ Yeast extract agar, Phosphoric acid, micro spatula, balance, Nichrome needle

Methodology - A small portion of the soil sample containing targeted hyphomycetes is transferred using a micro spatula into a sterilized Petri-dish. Then, $8-10 \mathrm{~mL}$ of agar medium in liquid form $\left(35-40^{\circ} \mathrm{C}\right)$ is added to the Petri-dish and soil particles are dispersed throughout the media by shaking and rotating the plate slightly (Clark et al. 1963, Wanderley-Costa et al. 2006). The amount of soil used in the preparation of a soil plate varies with the soil texture and collected locality. It has been experimentally proven that using $0.005-0.015 \mathrm{~g}$ of soil on Czapek-Dox +0.5 $\%$ yeast extract agars acidified with phosphoric acid of $\mathrm{pH} 4.0$ is ideal for the growth and sporulation of many soil fungi. A micro-spatula made by flattening the end of a Nichrome needle has been used for transferring and crushing soil aggregates (Ayse-Dilek 2003).

\section{Isolation of aquatic hyphomycetes}

Fisher et al. (1991) proposed a method to isolate aquatic hyphomycetes. This is similar to the direct streaking method. However, this method differs in inducing sporulation due to their survival in the aquatic environment.

Materials required $-75 \%$ ethanol, $0.93-1.3 \mathrm{M}$ solution of sodium hypochlorite (3-5\% available chlorine), $1.5 \%$ malt extract agar, antibiotics, distilled water, sterilized needle

Methodology - First, the substrate of hyphomycetous fungi should undergo surface sterilization process using $100 \%$ ethanol for 60 seconds, $0.93-1.3 \mathrm{M}$ solution of sodium hypochlorite (3-5\% free $\mathrm{Cl}_{2}$ ) for 3 min and $75 \%$ ethanol for 30 seconds. Then, the sterilized host specimen is cut into small pieces using a razor blade and placed on $1.5 \%$ malt extract agar (MEA) supplemented with antibiotics (Fisher et al. 1991).

\section{Isolating techniques for coelomycetes}

Coelomycetes are conidia-bearing fungi and the conidia are formed within a cavity lined by fungal tissue, host tissue or combination of both (Sutton 1980, Wijayawardene et al. 2016, Li et al. 2020). Conidia of coelomycetes are often exuded from the conidiomata and they should be isolated singly using different techniques.

\section{Single conidial isolation}

Single conidium isolation simply means that a single, germinating conidium is transferred to a pure media plate (Davis 1930). This ensures that the colony derived from a single, pure conidium and there is no mixing of genetic material from multiple conidia. Single conidium isolation is a basic and accurate method to obtain a pure culture. The single conidial technique is performed with pour plating, spread plating or streaking on the plate (Zhang et al. 2003). A sufficiently diluted pure conidia suspension is used, as this separates conidia well allowing for easy picking. 
In this technique, it is important to confirm that only target conidia have been isolated because one specimen can host more than one fungal taxon and fast-growing species often suppress others during isolation. Frequently, endophytes are also isolated along with targeted fungi (Ju et al. 2009). Therefore, initially, the surface of the specimen should be sterilized using $70 \%$ ethanol. Single conidium is obtained with a capillary pipette from a conidial suspension (Fig. 17) or direct picking using forceps under the dissecting microscope (Booth 1971).

Materials required - Fine tip forceps, needles, Pasteur pipette, flamed or cavity microscope slide, distilled water (all these materials should be sterilized using autoclave before using them), an alcohol lamp, $70 \%$ ethanol, fresh culture media plate $(90 \times 60 \mathrm{~mm})$.

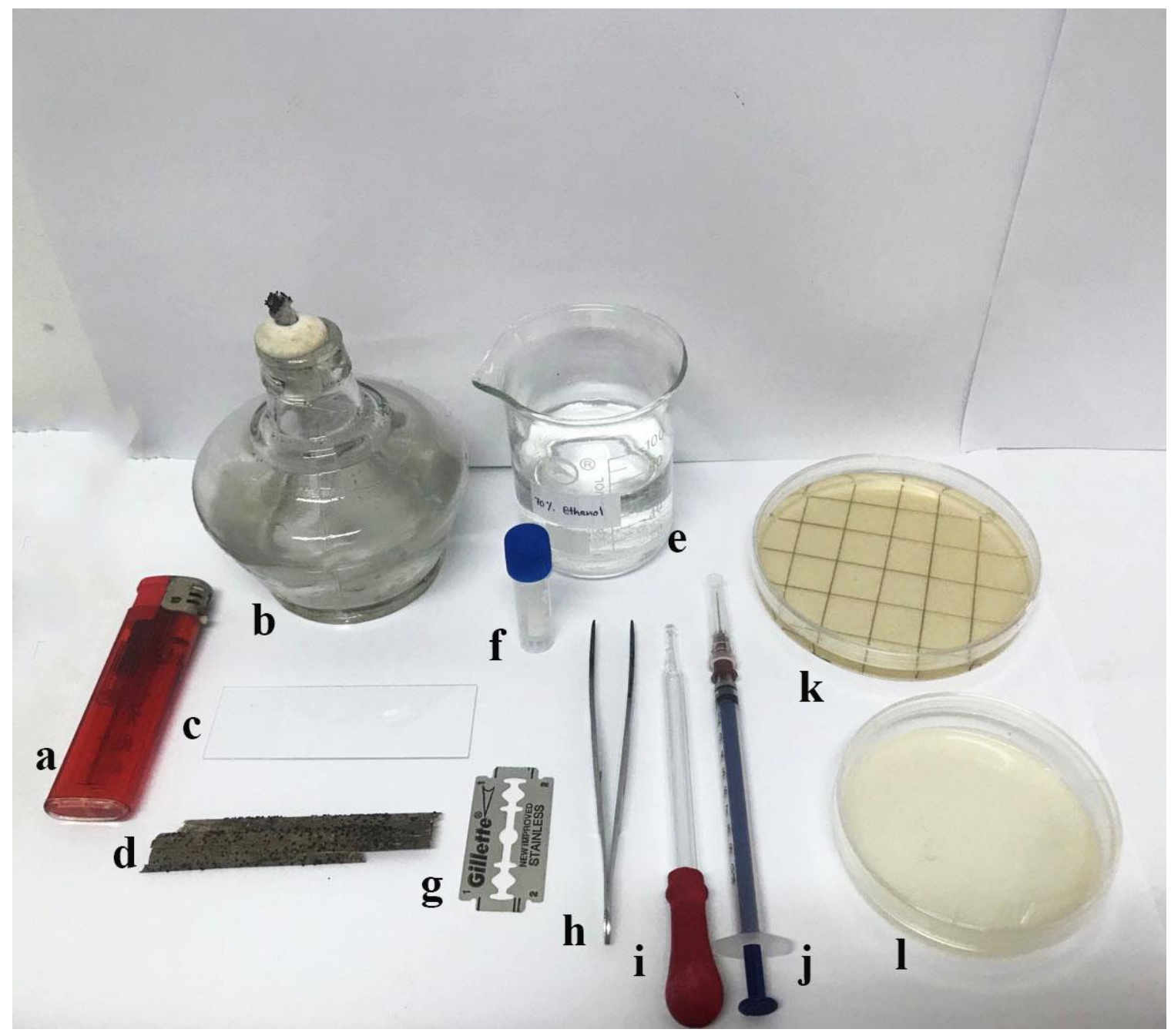

Figure 17 - Material and tools used in single conidium isolation. a Lighter. b Alcohol lamp. c Microscope slide. d Plant materials. e 70\% ethanol. f Sterilized distilled water. g Razor blade. h Forceps. i Pasteur pipette. j Needle with syringe. k $90 \mathrm{~mm}$ Petri-dish with marked squares. 160 mm Petri-dish.

Methodology - All tools and distilled water are sterilized before use. If necessary, antibacterial chemicals can be added in the culture media while cooling (at a temperature of less than $40^{\circ} \mathrm{C}$ ). All Petri-dishes are kept in laminar air flow to dry the water drops in the upper lid after pouring of culture media. The working space should be cleaned with $70 \%$ ethanol and two alcohol lamps are used to obtain aseptic conditions. For the spore suspension, a glass cavity slide is sterilized with $70 \%$ ethanol and wiped with a paper towel. A few sterilized water drops are placed on a glass cavity slide using a sterilized Pasteur pipette. Several conidiomata are picked from the surface-sterilized sample using sterilized fine-tipped forceps or the selected conidiomata are cut laterally using a razor blade. Conidial masses are transferred into sterilized water drops on the 
cavity slide using a sterilized needle. The water drop with conidial mass is examined under a dissecting microscope to confirm that sufficient conidia of interest have been transferred. The agitated conidial suspension is sucked into a sterilized Pasteur pipette. Small drops of conidial suspension are placed on the media. These media plates are incubated for 12-24 hours at room temperature or appropriate temperature. Plates should not be sealed to facilitate drying out of some of the surface water. If the plates are sealed with parafilm, water vapors will accumulate on the media surface and the chance of contamination will increase. However, if the plates are sealed with parafilm, they should be kept inverted. Plates should be observed after 12 hours and from then on every 24 hours for two days. Germination of conidia can be seen under a dissecting microscope. The agar surrounding the germinated conidia are carefully cut using a sterilized needle and transferred to new media plates. Two to four germinated conidia with germ tubes should be transferred to one media plate. Plates should be observed under the light microscope to confirm whether spores form colonies.

\section{Spore suspension method by vortex}

In this method, a spore suspension is prepared using a vortex mixer (Leyronas et al. 2012, Fei et al. 2019). Vortex mixer facilitates the breakdown of the ascus wall and releases spores easily to the suspension (Figs 18, 19).

Materials required - Micropipette and tips, fine forceps, alcohol lamp, fresh culture media plates (PDA), centrifugal tubes, vortex.

Methodology - Fruiting bodies are picked from the surface of sterilized plant material and placed on a sterilized microscopic slide. Then, fruiting bodies are crushed carefully using forceps under the dissecting microscope and transferred in a centrifuge tube with sterilized water. The centrifuge tube is covered quickly and vortexed to obtain a homogeneous spore suspension. A single drop of spore suspension is transferred onto each marked square on PDA using a micropipette under the laminar air flow and plates are sealed using parafilm. Inoculated media plates are incubated at appropriate temperatures.

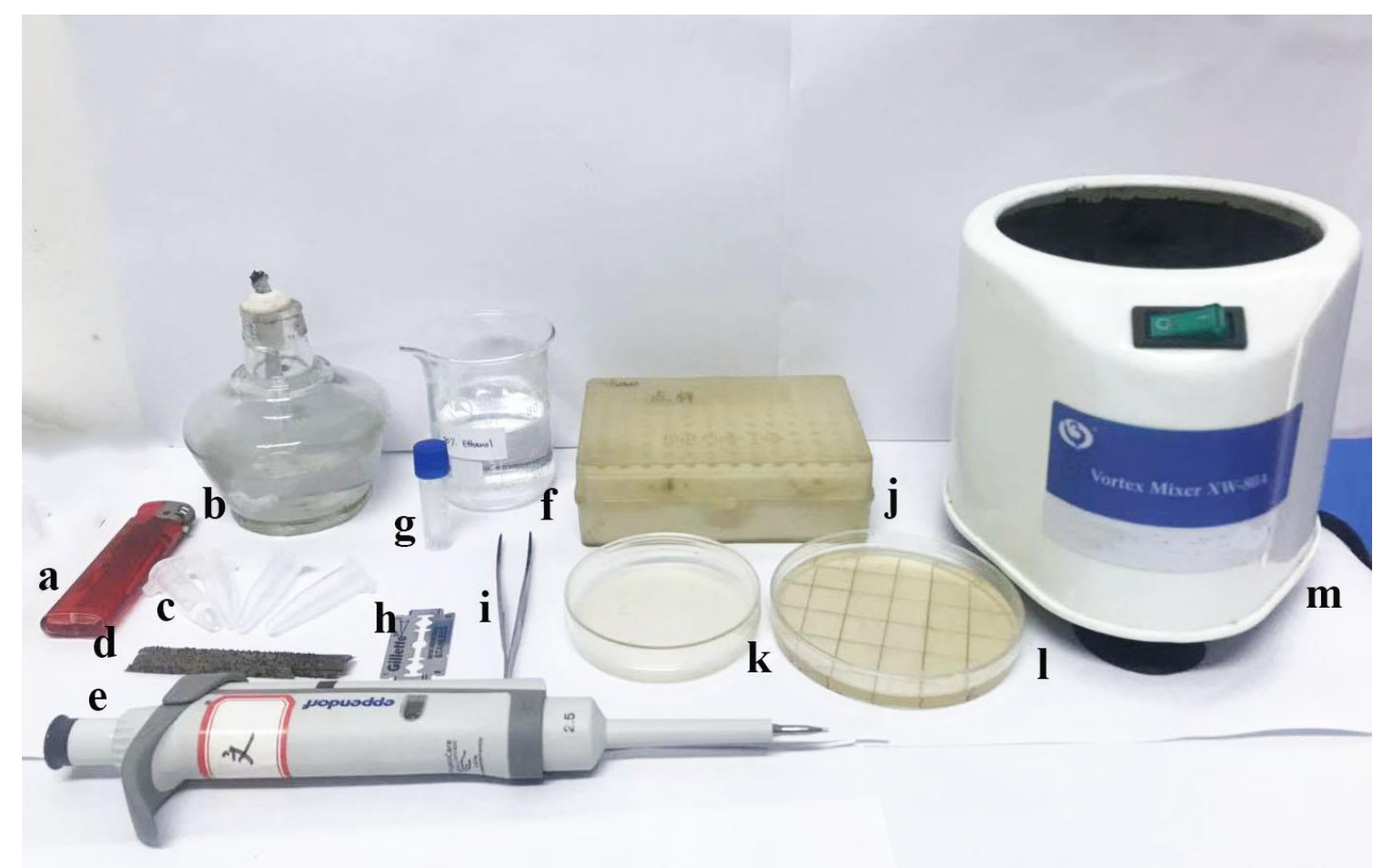

Figure 18 - Material and tools used in the spore suspension method. a Lighter. b Alcohol lamp. c Centrifuge tube. d Plant material. e Micropipette. f $70 \%$ ethanol. g Sterilized distilled water. 
h Razor blade i Forceps. j Micropipette tips. k 90 mm Petri-dish with media marked squares in lower surface. $160 \mathrm{~mm}$ Petri-dish. m Vortex.
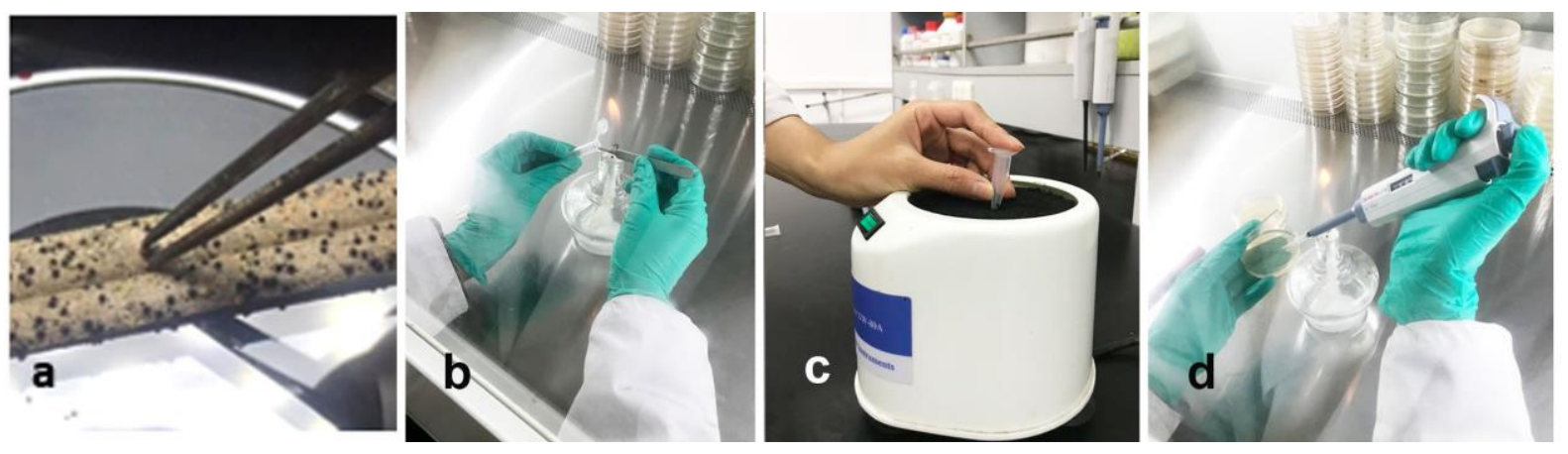

Figure 19 - Basic steps in spore suspension method. a Use of extra fine forceps to pick up fruiting bodies. $\mathrm{b}$ Transferring crushed fruiting bodies into sterilized water in a centrifugal tube. c Stirring suspension in centrifugal tubes using a vortex. $\mathrm{d}$ Use of micropipette to transfer suspensions onto agar plates.

\section{Isolating techniques for sexual morph of ascomycetes}

Ascomycetes produce perithecia, pseudothecia, cleistothecia or apothecia as sexual fruiting bodies (Pöggeler et al. 2006). These reproductive organs may be superficially immersed in the host surface. During isolation, fruiting bodies are opened and ascospores are taken out. Herein, several relevant techniques are discussed.

\section{Spotted pour technique}

The spotted pour technique is commonly used for isolation of the single germinating spore (Choi et al. 1999). A spore suspension is prepared as described above. Sixteen squares are marked on the bottom of the Petri-dish using a marker. One drop of homogenous spore suspension per square is transferred onto the media with a sterilized pipette (Choi et al. 1999). Individual drops are checked using a stereo-microscope to confirm that the correct fungus has been selected (Hildebrand 1938). Plates are sealed with parafilm and kept inverted to prevent water accumulation on the agar surface. Plates are incubated at $18^{\circ} \mathrm{C}$ to $25^{\circ} \mathrm{C}$ for $12-24$ hours (Fig. 20).

Spores are checked within 12-24 hours for germination. Upon germination, a very small amount of agar surrounding the germinating spore can be cut with a very fine sterilized needle and transferred to fresh media (Booth 1971). Selecting germinating spores with relatively short germ tubes prevents the transfer of excess agar. Germinating ascospores can be easily recognized, when observed using a stereo-microscope at the highest magnification or compound microscope using 10-20 times lenses. It is better to transfer spores to several other plates to keep replicates. Spores should be transferred to new media within an hour after germination. Otherwise germinating spores form colonies and this may result in picking up spores from different species. In addition, spore wall disappear when the germ tube develop into mycelia and it is difficult to select the correct germ tubes to pick up (Fig. 21).

\section{Multiple spore or asci isolation}

Multiple spore isolation can be obtained for taxa which produces a large number of spores. This is a perfect isolation method for fungi that produce small spores and whose germinating spores are not clearly visible (Stadler et al. 2014). In addition, hyaline spores are also difficult to see on culture media plates. Therefore, multiple spore isolation may be applicable in this situation (Bills et al. 2012). In this technique, the fruiting body on sterilized host is horizontally cut using a sterilized blade and the perithecial content is streaked on a media plate (Wongkanoun et al. 2019). Germination of asci or multiple spores can occur on media plates (Fig. 22). 


\section{Direct isolation from fungal tissues}

Sometimes, collected specimens are not in good condition and spores may be either overmature or present in low numbers. In these cases, subsisting parts of peridium, hamathecium and vegetative hyphae can be used to obtain a culture (Rozas et al. 2011). If there are any mature spores attached to peridium or hamathecium content, they may germinate and produce the colonies. For this process, tissues are rehydrated before being transferred to the media (Liu et al. 2015).

Materials required $-70 \%$ alcohol, sterilized tissues, fungal specimen, sterilized blade, forceps, fresh media plates, parafilm

Methodology - A small piece of surface sterilized ascoma is cut away from the substrate. These vegetative fungal pieces are rehydrated with a few drops of sterilized water. The pieces of ascomata are transferred to fresh media plates using sterilized forceps and plates are incubated and checked for germination after 24 hours.

\section{Spore shooting or drop method}

Spore shooting or spore drop method can be used to isolate ascomycetes (Choi et al. 1999). Some fungi produce huge spore masses and spores are released through a long neck. Some species eject the spores and spore shooting or drop method can be applied. For ascomycetes, ascospore should be ejected from the fruiting body (Choi et al. 1999).

Material required - fine-tipped forceps, needle, wax, fresh culture media plates, razor blade, sterilized scalpel, fungal specimens.

Methodology - A few, surface sterilized, fresh ascomata picked from the substrate or a small piece of the substrate containing ascomata are and soaked in sterilized, distilled water for 2 hours. The fruiting bodies are attached to the inner surface of the upper lid of media and wrapped with parafilm. Plates are incubated vertically (Fig. 23) and are checked for the spore germination after 24 hours.

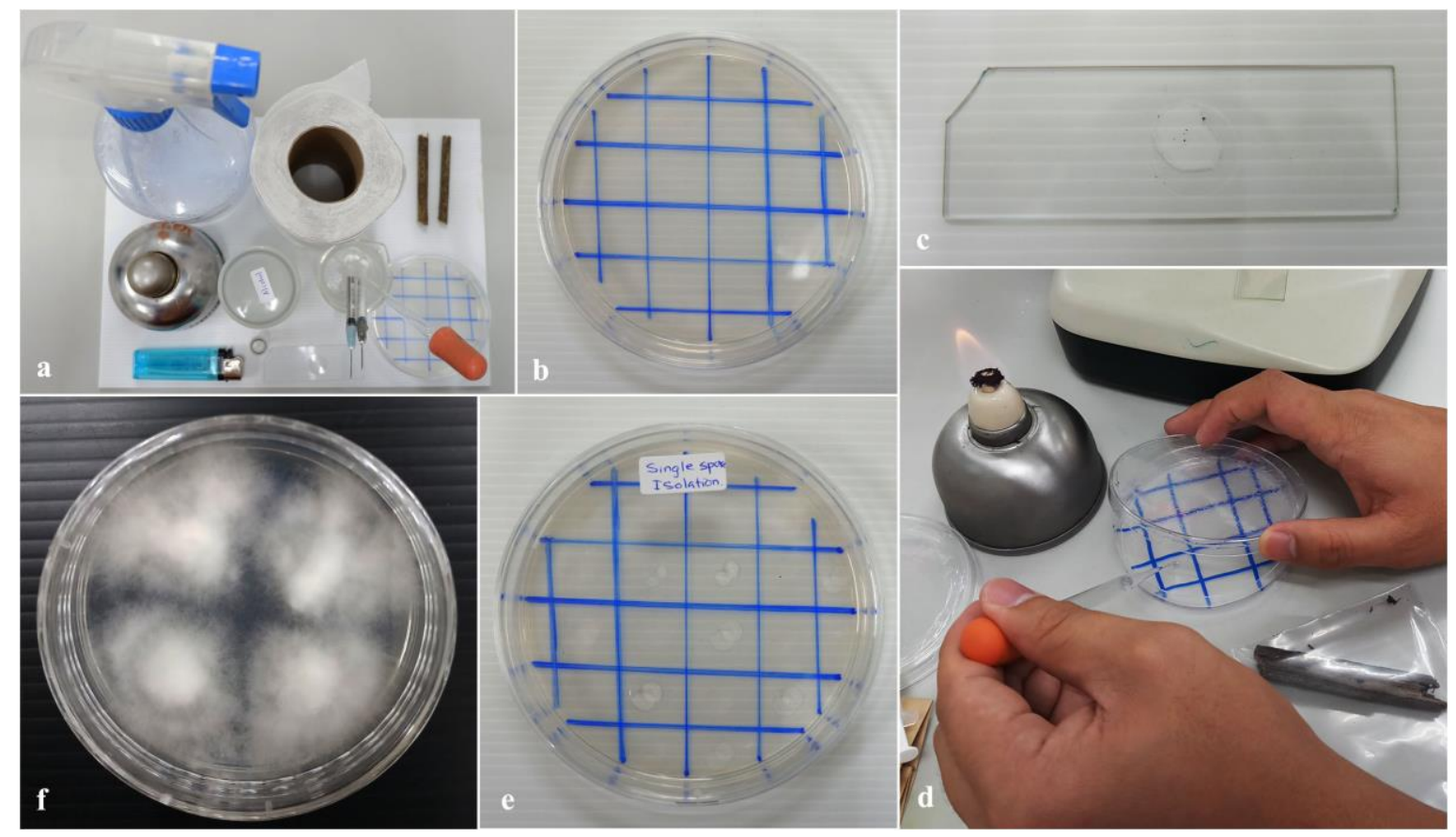

Figure 20 - Single spore isolation by spotted pour technique. a Tools. b Squares on bottom of the PDA plate. c Spore suspension. d Spore transfer. e Labeling. f Culture from transferred germinated spores.

\section{Isolating techniques for epiphytes}

Fungal epiphytes live on plant and leaf surfaces and can be categorized as obligate parasites or saprobes (Hyde et al. 2013, Li et al. 2016). Saprobic epiphytes produce a black coating of 
hyphae on the leaf and fruit surface on economically valuable crops and they reduce the photosynthetic ability (Reynolds 1998). The hyphal coating can also cause chlorosis (Ismail et al. 2016). Hyphae of multiple sooty mold species are combined in blackened superficial colonies on the host and the morphology of the hyphal network gives taxonomic information of various species (Hughes 1976, Hughes \& Seifert 2012, Chomnunti et al. 2014). Therefore, careful isolation of epiphytes is needed for reliable phylogeny based classification.
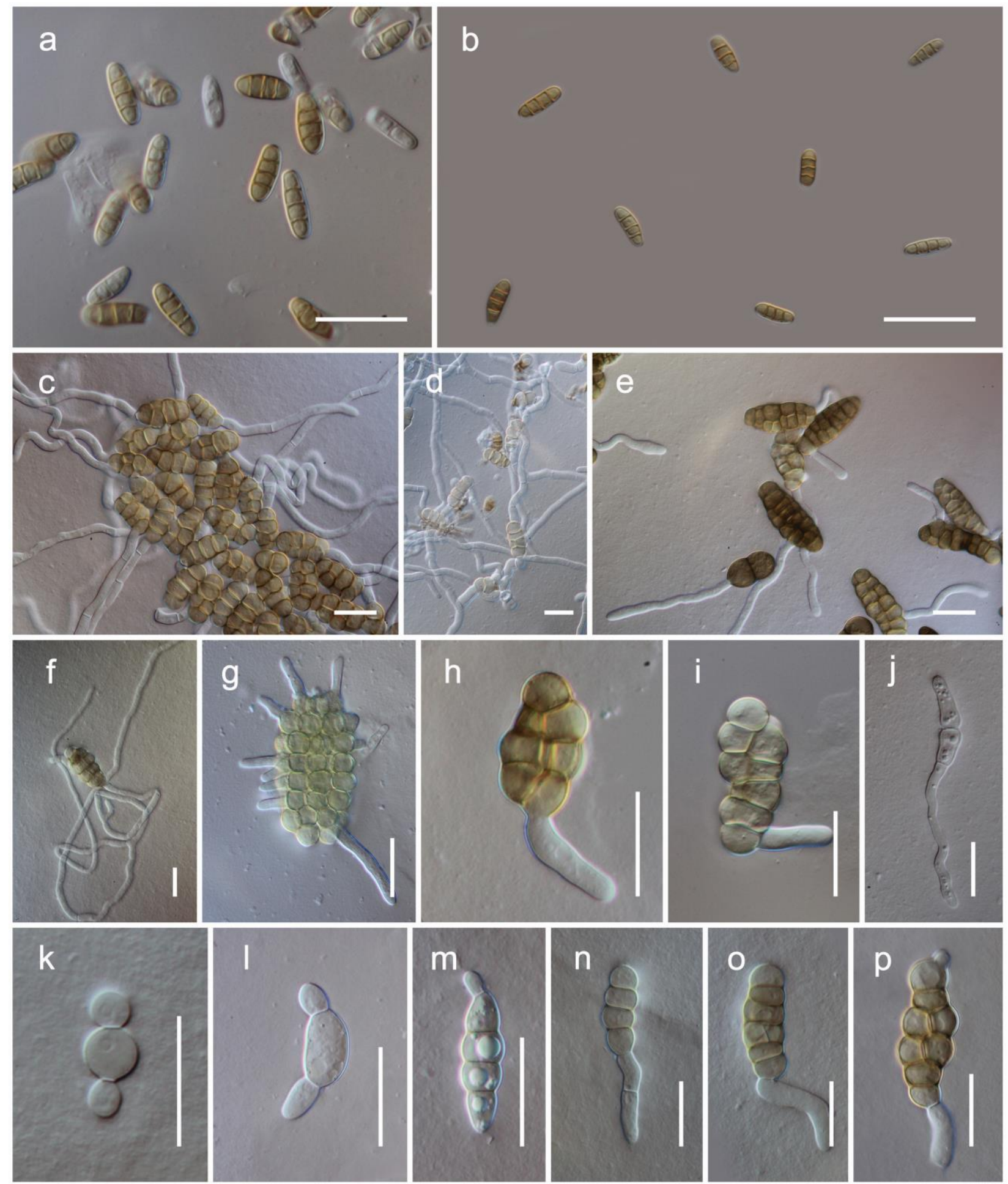

Figure 21 - Spore density on media and germinating spores. a Too crowded spore spread. b Welldistributed spore spread. c Germinating spores in crowded spore spread. d Over grown germ tubes formed mycelia. e Spore suspension with different spores. $f$ Overgrown germ tubes. g-p Germinating spores suit to transfer. 


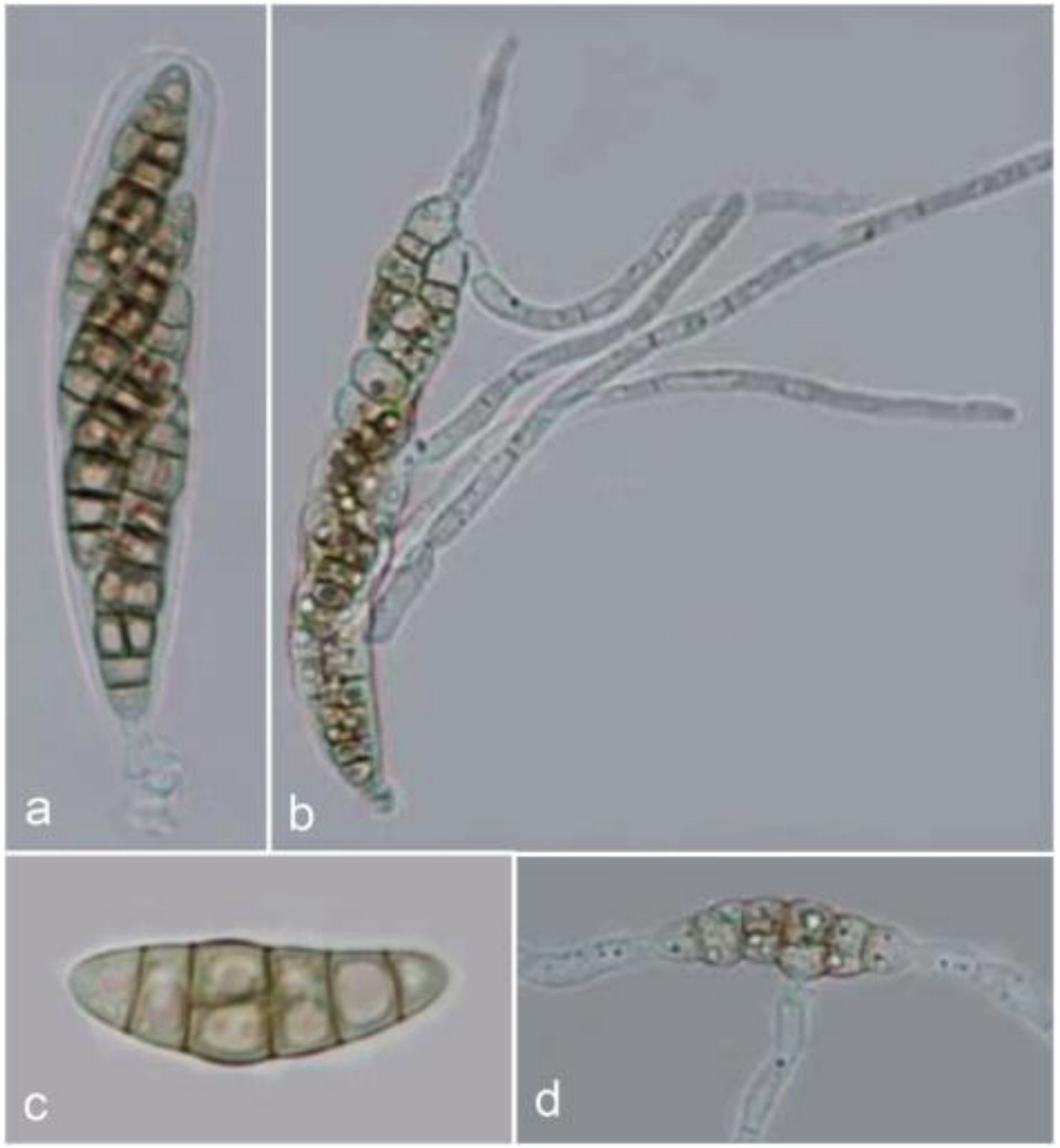

Figure 22 - Different forms of germinating single or multi ascospores; ascospores and asci of Phaerosphaeria vagans.
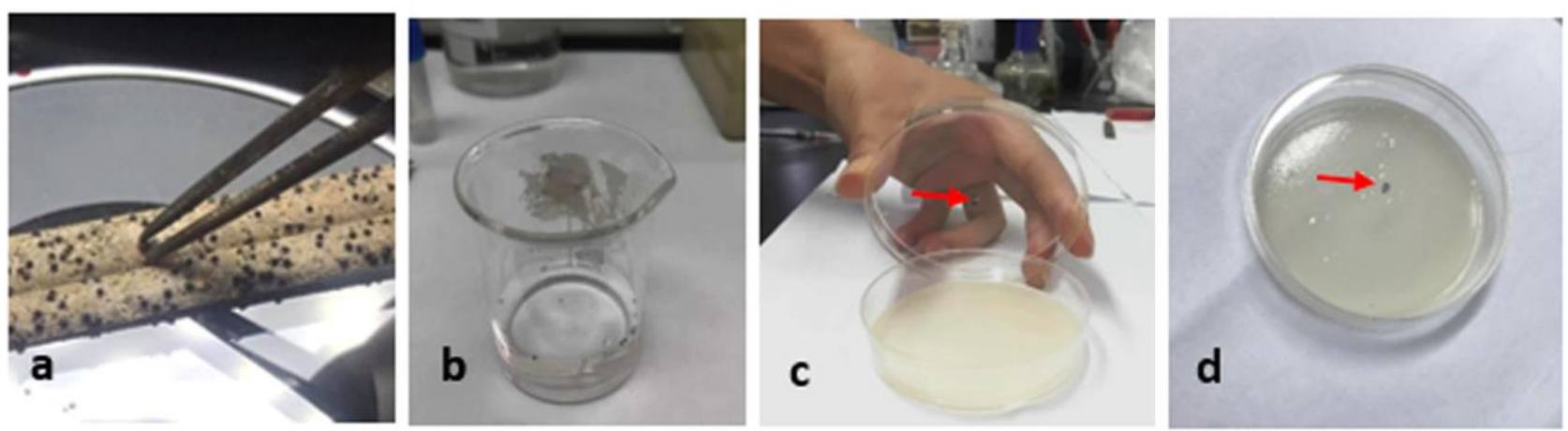

Figure 23 - Spore shooting method. a Picking up fruit-bodies using extra fine forceps. b Soaking the picked fruiting bodies in sterilized distilled water. c Conidiomata in the Petri-dish lid (arrow showing place of conidiomata on lid). $\mathrm{d}$ Incubation of plates for $24 \mathrm{hr}$ at room temperature. 


\section{Imprint method}

This method was proposed by Santamaría \& Bayman (2005). Leaf imprinting on agar was used to isolate the epiphytic fungi from leaves (Fiss et al. 2000).

Materials required - Specimen, fine forceps, surgical blade, sterilized distilled water, glass Petri-dish, sterilized filter papers, Petri-dish with media, alcohol burner and lighter, $70 \%$ alcohol, tissue, marker pen, wrapping tape, labels.

Methodology - The working bench is cleaned using $70 \%$ alcohol. Then leaf specimens are cut into $5 \times 5 \mathrm{~mm}$ pieces. Leaf pieces are placed on the media plate as adaxial or upper leaf surfaces need to be in contact with the agar medium. Leaf pieces are removed after 1 hour and PDA plates are then incubated at $25^{\circ} \mathrm{C}$ for $12-24$ hours. Germinated spores are transferred to another media plate. After colonies grow, a small piece of mycelium with agar is cut and transferred to another media plate to obtain a pure culture.

\section{Isolation techniques for endophytes}

Endophytes are an endosymbiotic group of microorganisms often bacteria or fungi that colonize inter or intracellular locations of plants for completely or partially spending their life (Pimentel et al. 2011, Singh \& Dubey 2015). Culture based studies are regularly used in endophytic research (Raja et al. 2016). Therefore, isolation of endophytic fungi is necessary for their morphological characterization, studying population dynamics and species diversity, use culturebased inoculants to intensify plant development, and screening for novel naturally active secondary metabolites (Philipson \& Blair 1957, Hartmann et al. 2000).

Healthy leaves, stems and roots are plucked from selected plants (Jiaojiao et al. 2016, Kannan et al. 2017). Surface sterilization is the initial and important step for isolation of endophytes (Torres et al. 2011, Gan et al. 2017). Some plant tissues require additional pre-treatment as their exterior parts comprise hydrophobic materials such as waxy cuticle (Coombs \& Franco 2003, Radhakrishnan et al. 2013). Sanitation is necessary before surface sterilization to remove soil and organic particles from the plant parts (Coombs \& Franco 2003). The leaves, stems and roots of each plant are washed separately under running tap water to remove soil particles and the majority of microbial surface epiphytes (Richter-Heitmann et al. 2016, Saad et al. 2019). For the information on surface sterilization, Please see: Sanitation and surface sterilization of specimen.

\section{Plant tissue fragments culture method}

This technique is suited for isolating endophytic fungi and also actinobacteria (Golinska et al. 2015). Slow growing microorganisms are often underestimated during endophytic diversity studies (Hyde \& Soytong 2008). Also, the growth of two or more microorganisms may require parting of the isolates and may perhaps end up resulting in a low amount of colonies (Arnold 2007, Toghueo et al. 2017).

Material required $-1 \%$ Sodium hypochlorite $(\mathrm{NaClO}), 70 \%$ ethanol, sterile water, Petridishes, forceps, media plates, alcohol lamp

Methodology - The surface-sterilized plant parts are cut into fragments under aseptic conditions (Oses et al. 2008, Geisen et al. 2018). Fragments are placed directly on an appropriate nutrient medium (Rödel et al. 2016). Tissue fragments should be cut into very small and thin pieces as colonization may be inadequate (Fig. 24) (Anjum \& Chandra 2019, Hassan et al. 2019). The chosen nutrient medium is supplemented with antibiotics to prevent any unwanted microbial growth (Sanders 2012). Antifungal substances can also slightly be added to delay the development of fast-growing fungal contaminants (Müller \& Ruppel 2014, Mat-Jalil \& Ibrahim 2018). Endophytic microorganisms that arise from plant fragments are consequently shifted to fresh media (Bernardi-Wenzel et al. 2010).

Most endophytic fungi, especially if slow growing should undergo a soaking process (Sieber 2002). Hypothetically, this method traps endophytic invaders of the plant tissue, including colonizers of the root cortex or vascular tissue, intercellular as well as intracellular colonizers. To increase the efficiency of this process, sterile water or buffer solution is added to the surface- 
sterilized plant tissue before maceration (Chi et al. 2005, Mattos et al. 2008). This method is simple and reliable.

Material required - mortar and pestle or other automated machines such as Klecco tissue pulverizer, Polytron homogenizer or a blender, needle, culture media plates

Methodology - Surface sterilized plant parts are macerated with water. The duration and intensity of maceration need to be calculated. The temperature should be monitored and cooling is necessary to deactivate plant enzymes and toxins released during maceration. Otherwise, plant enzymes and toxins can deactivate or destroy the endophytic fungi (Hardoim et al. 2015, Hyde et al. 2019). After the maceration of plant tissue, the suspension is streaked on culture medium under aseptic conditions (Ramalashmi et al. 2018, Shukla \& Wahla 2019).

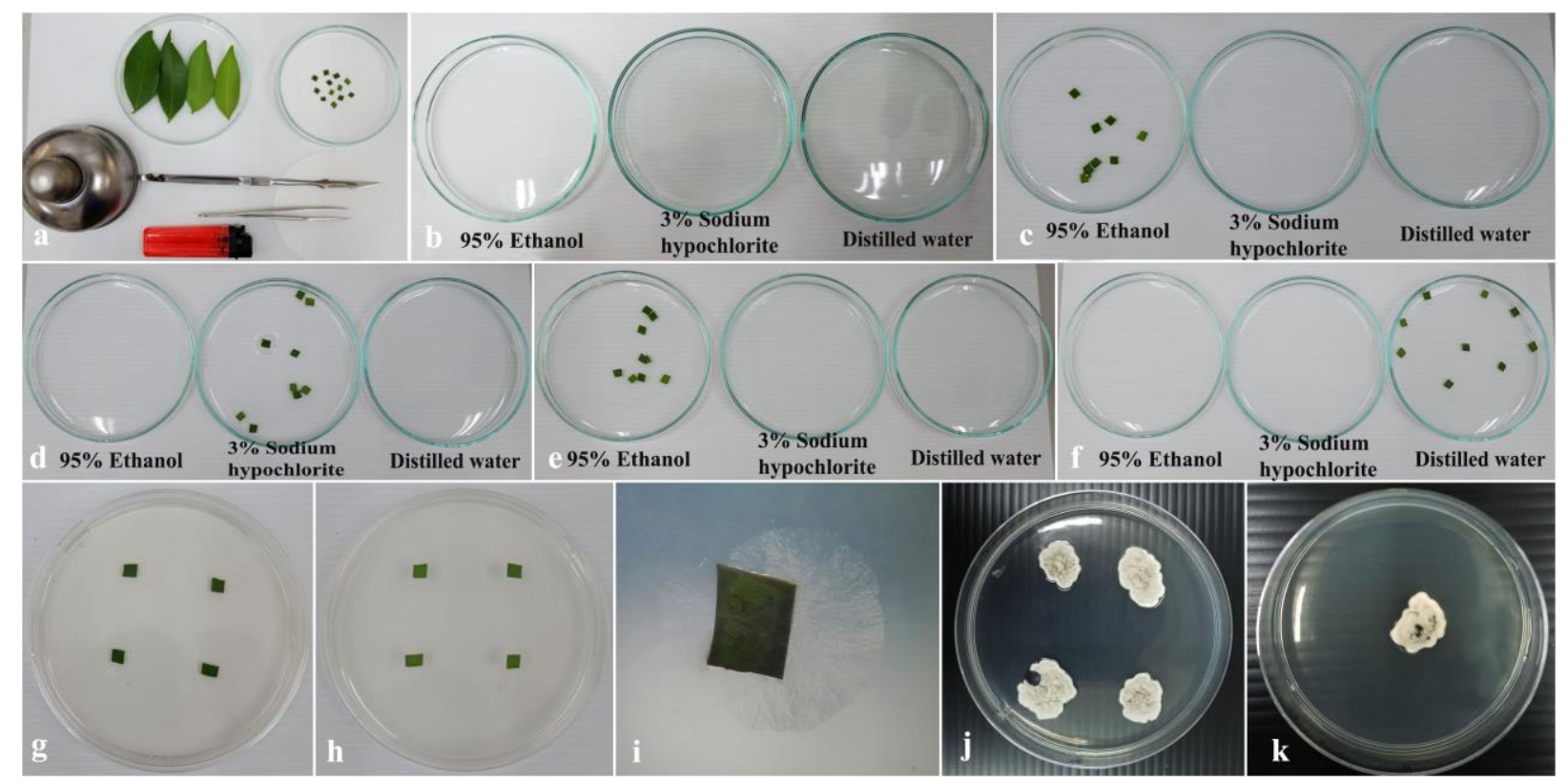

Figure 24 - Isolation endophytes by cultivating plant tissue fragments. a Tools. b Media for Surface Sterilization. c Soaking in 95\% ethanol. d Soaking in 3\% sodium hypochlorite. e Soaking in $95 \%$ ethanol. $f$ Washing in distilled water. $g$, $h$ Leaf pieces placed on a PDA plate. $g$ upper side of PDA plate. h lower side of PDA plate. i Colony growth on PDA (after 72 hours). $\mathrm{j}$ Colony growth on PDA (after 6 days). $k$ Pure culture.

\section{Plant tissue maceration method}

\section{Plant intercellular fluid culture method}

During centrifugation, the intercellular fluid of the plant tissue is collected to extract the endophytic fungi. The method has been used frequently and is especially successful for root tissues (McCully 2001, Tolulope et al. 2015, Gouda 2016, Hamayun et al. 2018, Shah et al. 2018, Wang et al. 2019). The advantage of this technique is that it avoids maceration of plant tissue, reduces contamination and can deal with several samples at the same time.

Material required - Centrifuge machine, centrifuge tube, $70 \%$ ethanol, sterile water, $1 \%$ sodium hypochlorite, blades, forceps.

Methodology - Plant tissues are washed with distilled water for 1 min., and then air-dried. The leaf surface is sterilized by dipping the leaves in $70 \%$ ethanol for $30 \mathrm{~s}$, followed by dipping in $1 \%$ sodium hypochlorite for $60 \mathrm{~s}$ and subsequently rinsing in sterile demineralized distilled water. The cleaned leaves are cut into pieces of approximately $0.5 \mathrm{~cm}^{2}$. Plant tissues are centrifuged at $3000 \mathrm{~g}$ and the apoplastic fluid is collected (Dong et al. 1994). The fluid streaked on the media for endophyte isolation. 


\section{Isolating techniques for plant pathogens}

The specimen or tissue parts used in isolating plant pathogens usually consist of the interphase or transitional area between healthy and infected tissue (Melotto et al. 2008). The choice of material used for isolation may depend on the experience of the mycologist. Depending on the pathogens, different techniques may be required for isolation (Abdulkhair \& Alghuthaymi 2016). General techniques such as single spore and tissue isolation are used to isolate commonly occurring fungi. In order to isolate primary pathogens, specific techniques such as baits or selective media may be used.

In all isolation methods, the Petri-dishes are maintained at room temperature or in an incubator at a specific temperature such as $25-30^{\circ} \mathrm{C}$. These cultures are typically maintained for $3-$ 10 days and are examined daily for microbial growth. The type of culture medium is an important factor when isolating plant pathogens. Potato dextrose agar (PDA) is the general culture medium that many plant pathogens can utilize. Acidified PDA suppresses bacterial growth while allowing fungi to grow (Trigiano \& Ownley 2017). However, some fungal pathogens may require selective media to grow as high carbohydrate and nitrogen content with a $\mathrm{pH}$ range of 5-6 and a temperature range from $15-37^{\circ} \mathrm{C}$ (Basu et al. 2015).

\section{Baiting techniques for isolation of soilborne plant pathogenic fungi}

Soilborne plant pathogenic fungi can be divided into two groups as root-inhabiting specialists that cannot grow in root free soil and specialists that are capable of growing through root free soil (Kariman et al. 2018). In both cases roots are sampled, thoroughly washed, surface sterilized, dried, dissected and plated on media that contain antibiotics.

Baits are used to trap and isolate plant pathogens (Fig. 25). Baits are usually a desirable plant host that can stimulate pathogen growth. For example, species of Phytophthora produce motile zoospores, which are the dispersal mode and cause new infection. In order to isolate Phytophthora species from a diseased plant root or from the surrounding soil, both root and soil are submerged in water and pieces of leaf disks can be floated on the water surface. Zoospores of Phytophthora will be attracted to the leaf disks and after several days the disks are carefully removed from the water, blotted dry and placed on a semi-selective medium. If species of Phytophthora are present in the diseased root or soil, growing mycelium from the leaf disk will be observed.

\section{Direct isolation for above ground plant pathogenic fungi}

If fruiting bodies are clearly visible on symptomatic plant tissues, then plant material is incubated for 24-48 hours in a moist chamber and single spore isolation is used to obtain a pure culture (Ampt et al. 2019). Plant pathologists use this method with modifications. For example, if parts of plant tissue containing several mature fruiting bodies can be placed in a tube of sterilized water until enough spores are counted in the suspension, this can be plated out (Fig. 26) (Schuck et al. 2014).

\section{Indirect isolation for above ground plant pathogenic fungi}

Poorly or non-sporulating pathogenic fungi have to be isolated indirectly from symptomatic plant tissues. These plant tissues are usually surface sterilized to minimize contaminants or other saprobes that might be present. The reason is the pathogen causing the disease would be present in the infected and damaged internal tissue (Bolton \& Thomma 2012).

Material required $-70 \%$ alcohol, $10 \% \mathrm{NaOHCl}$, sterile water, tissue papers, culture media plates, forceps,

Methodology - Surface sterilization is done by soaking the plant tissues in $70 \%$ alcohol and in $10 \% \mathrm{NaOHCl}$ solution. The exposure time of the plant material to the sterilization agent can vary from a few seconds to several minutes, depending on the permeability of the plant tissue. Once plant tissues are sterilized with chemicals, they are rinsed in sterilized distilled water. Excess water is blotted off with sterilized blotting paper and allowed to air dry. Throughout this process, plant materials should be handled aseptically. Then, the plant tissue is sectioned into small pieces and 
placed on appropriate media in Petri-dishes. If the surface sterilization was done correctly and if there is a pathogen in the plant material, fungal growth originating from internal parts of the plated plant tissue can be seen and the cultures recovered will be morphologically consistent (Fig. 27).
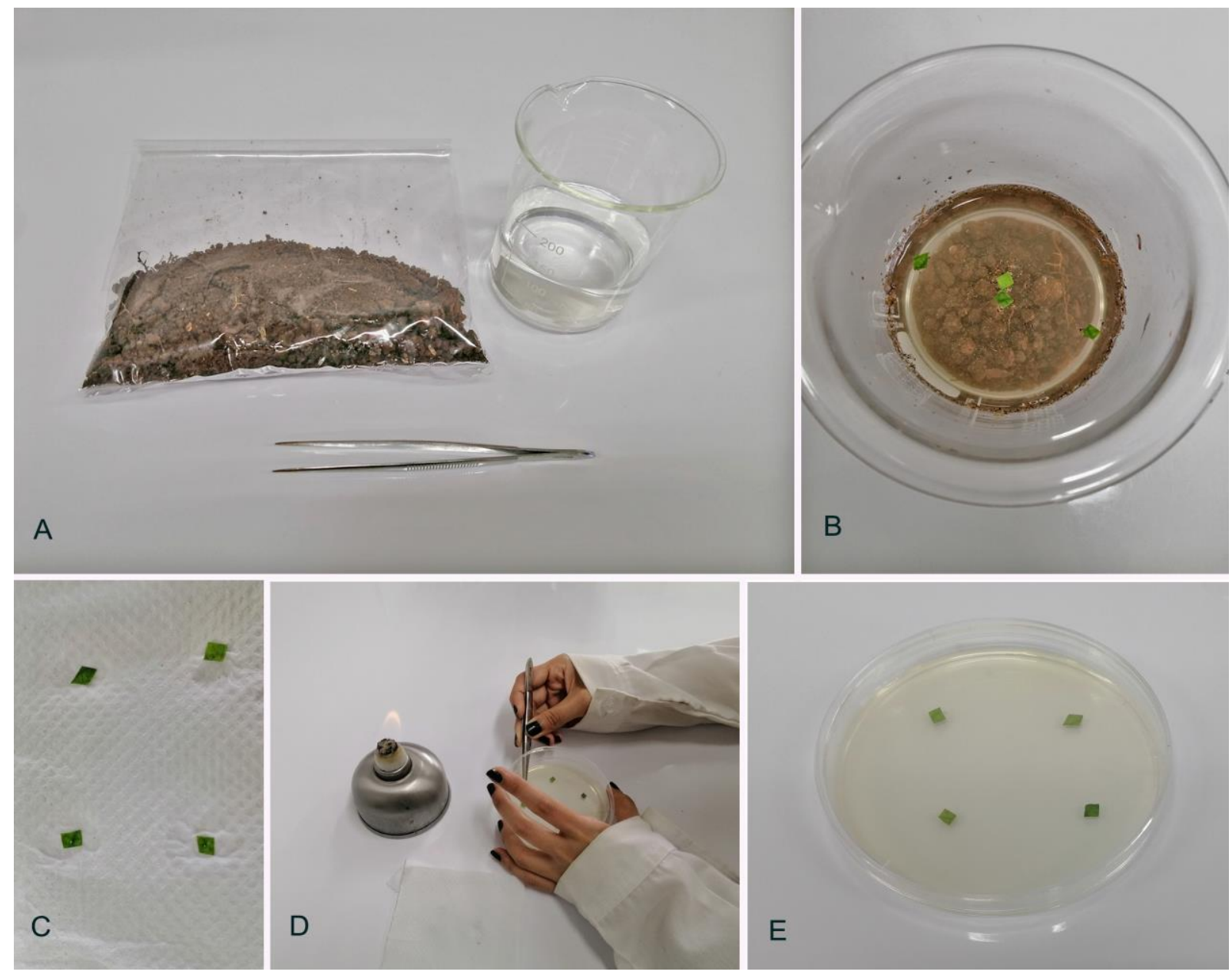

Figure 25 - Baiting technique for plant pathogens. a Soil sample, sterilized water and sterilized forceps. b Soil sample submerged in water with pieces of the bait (eucalyptus, Camelia sp. or Rhododendron sp. leaves) floated on the water surface. c Leaf disks blotted dry. d Leaf disks placed onto the suitable medium in aseptic condition. e Leaf disks onto medium.

\section{Indirect isolation from fruiting bodies}

Poorly or non-sporulating fruiting bodies or mycelium fragments can also be transferred directly onto agar (Zhang et al. 2013). In this method, fruiting bodies are squashed and fragments are streaked on a fresh culture plate (Fig. 28).

Material required - needles, culture media plates, forceps

Methodology - Surface sterilized plant parts are cut into small pieces. Small plant parts are held using forceps and streaked on to media plates. If the samples are fruiting bodies, then, they are squashed and streaked on the media plates.

\section{Spore shooting method}

Spore shooting is another indirect method for isolating plant pathogens. Material required and methodology is noted in: Isolation techniques for coelomycetes (please see page 36).

\section{Isolation techniques for soil fungi}

Many methods have been used to isolate fungi from soils to estimate fungal diversity (Warcup 1960). The hyphal isolation method has been used to assess active mycelial forms in soil (Warcup 1955, Parkinson \& Williams 1960). Soil-washing technique has been used to isolate 
microfungi on root surfaces, organic materials, decaying wood and leaf litter (Simmonds 1930, Robertson 1954, Harley \& Waid 1955, Thorn et al. 1996). However, ascomycetous and zygomycetous fungal spores were removed by this method. Therefore, an immersion-tube technique has been used to isolate ascomycetous and zygomycetous taxa from soil (Chesters 1940).

Soil fungi can be isolated directly from scattered soil particles or spores and mycelial fragments in soil suspension (Chesters 1940). Soil is rich in fungal diversity (Gams 1992) since saprophytic fungi are the major recyclers in litter and soil (Thorn et al. 1996). Warcup's soil plate method is the most successful isolation method for saprophytic soil Zygomycetes (Steiman et al. 1995, Thorn et al. 1996).

\section{Soil dilution plating method}

Soil samples are diluted to reduce the density of fungi in each dilution tube (Aziz \& Zainol 2018). This dilution method was adopted by Waksman (1927) and upgraded by Aziz \& Zainol (2018).
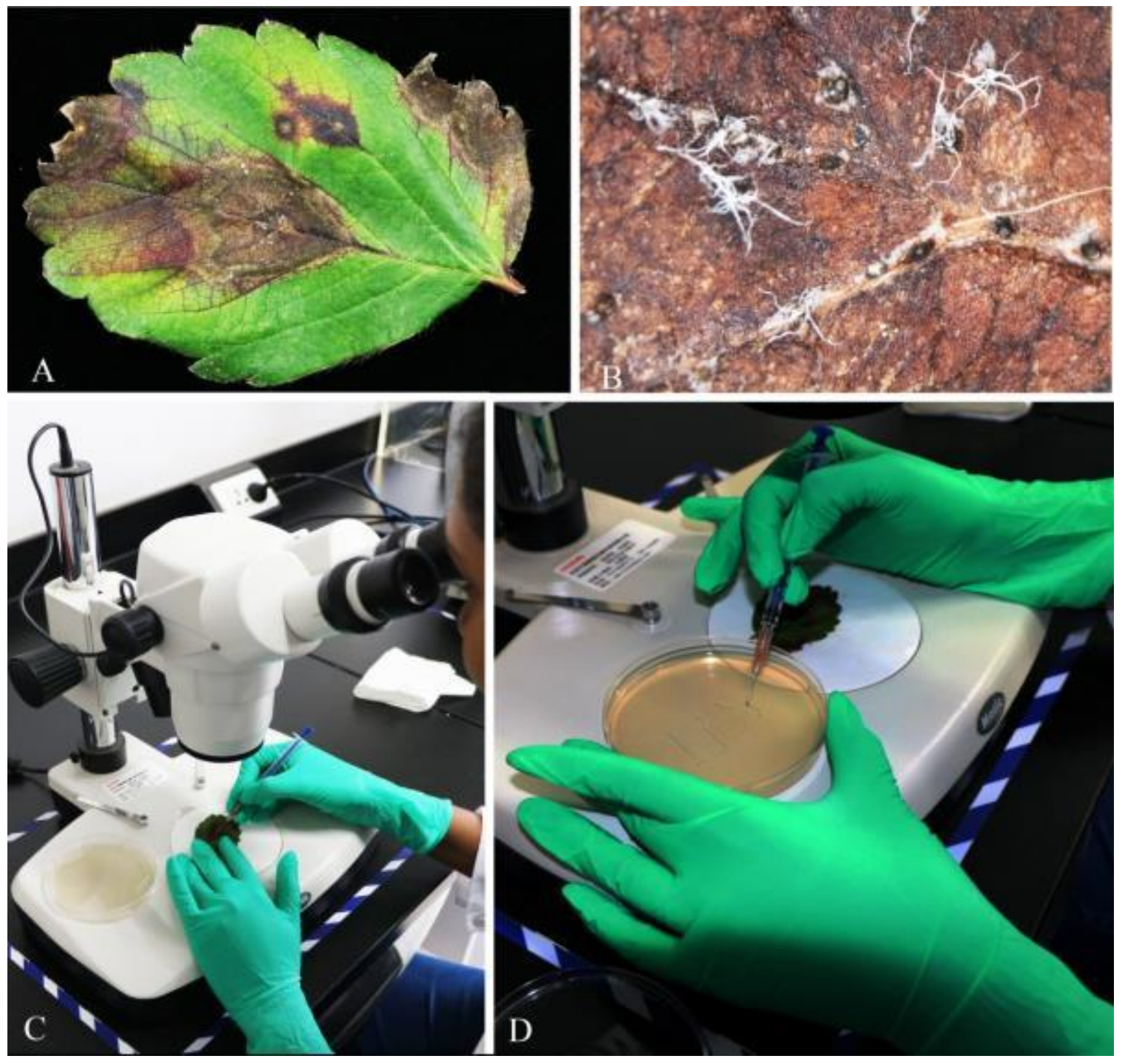

Figure 26 - Direct isolation from fruiting bodies (include Botrytis and any other oozing fruiting bodies on a host). a Disease symptom on leaves. b Fruiting bodies on substrate. c Picking fruiting bodies under a stereomicroscope. $\mathrm{d}$ Streaking on media plates. 

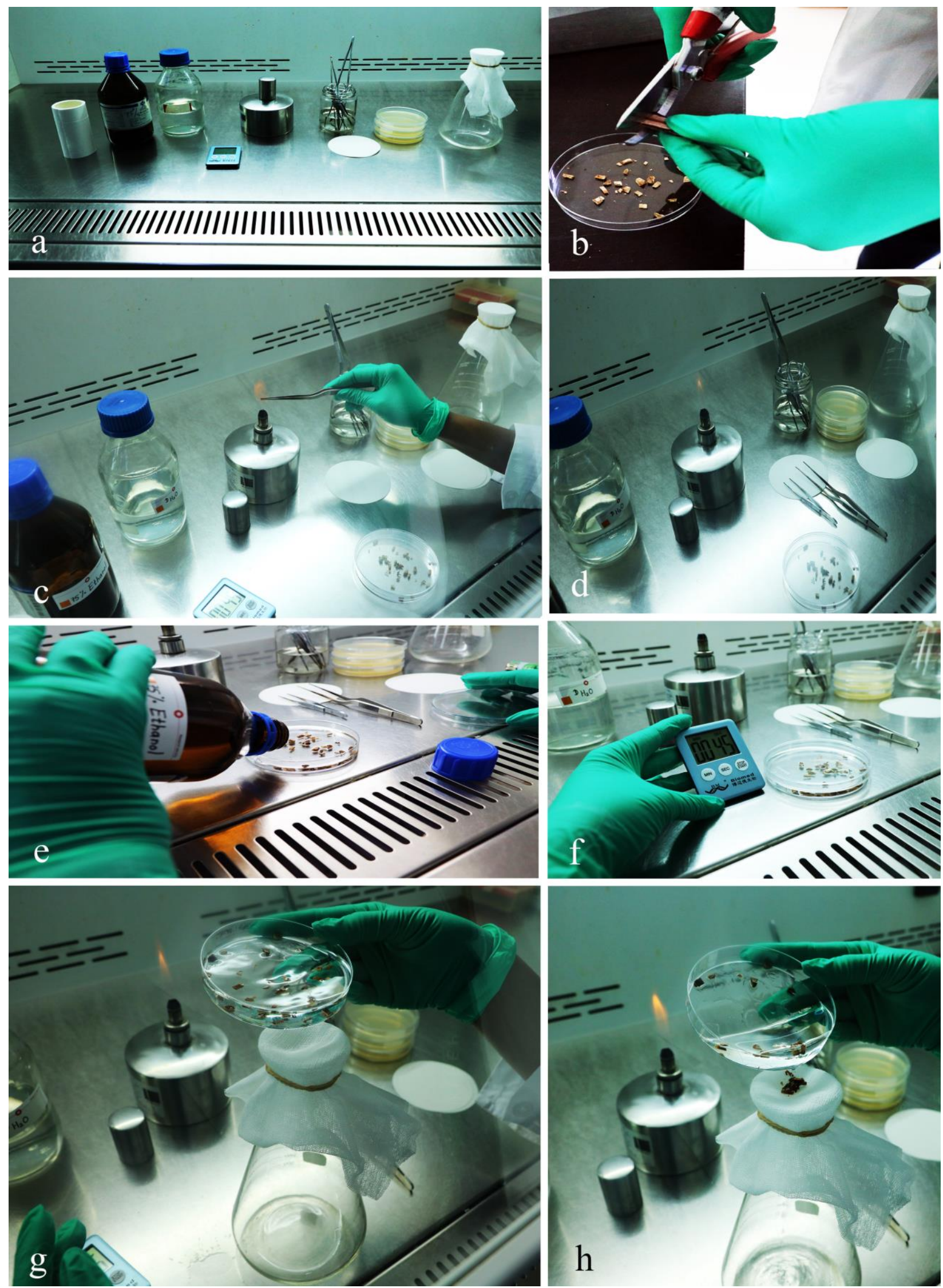

Figure 27 - Indirect isolation from tissue. a Tools (75\% ethanol, Distilled water, media plates, burner, timer, blotting papers and forceps). b Tissues cut into small pieces. c, d Heat sterilization of forceps. e, f Surface sterilization of tissues using $75 \%$ ethanol. g, h Removal of used $75 \%$ ethanol and drying of tissue pieces. 

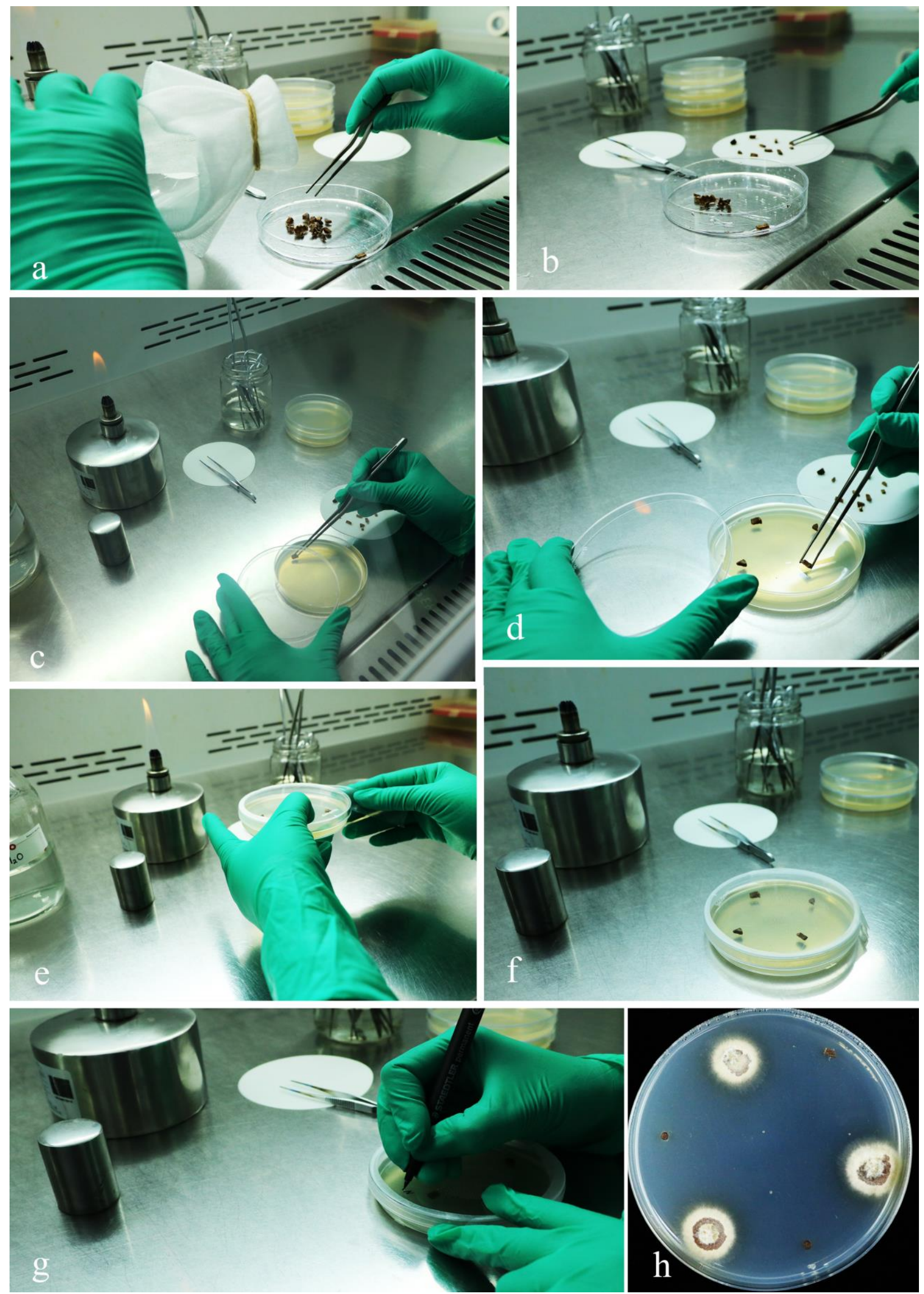

Figure 28 - Indirect isolation from fruiting bodies. a, b Air dried surface sterilized tissues. c, d Dried tissues placed on the media plates. e, f Sealed Petri-dishes. g Labelled plates. $\mathrm{h}$ Incubation of media plates to observe any mycelium growth. 
Material required $-70 \%$ alcohol, alcohol lamp, dissecting microscope, lighter, $85 \% \mathrm{NaCl}$ solution or distilled water, fine forceps, tissue papers, wrapper, PDA media plates, $1 \%$ streptomycin solution, test tube series

Methodology - Soil samples just below the litter layer, from different habitats are collected using a sterilized cork borer. Samples are stored in sterile polyethylene bags until processing in the laboratory at $48^{\circ} \mathrm{C}$. Approximately, $2.5-4.5 \mathrm{~g}$ of dry soil sample is added in $500 \mathrm{~mL}$ of sterile $0.1 \%$ sodium pyrophosphate. The aqueous mixture is intermittently shaken for 1 hour at $48^{\circ} \mathrm{C}$ for dispersing clumps and colloids of soil. The whole suspension is sieved through a soil sieve series (Thorn et al. 1996). The final dense suspension is air-dried and $50 \mathrm{~g}$ of dried soil suspension is mixed with $100 \mathrm{~mL} \mathrm{85 \%} \mathrm{NaCl}$ solution and shaken well (Ratna Kumar et al. 2015). The mixture is diluted from $10^{-1}$ to $10^{-6}$. Finally, $0.1 \mathrm{~mL}$ from each vial is pipetted into an antibiotic added culture media plate. Plates should be observed for 4-6 weeks. Colonies are examined under a microscope for the production of conidia and clamp connections at the septa. Diazonium blue B turns clamp connections red to purple which aid for identification (Thorn et al. 1996).

\section{Soil plate method}

The soil plate method was developed by Warcup (1950) to isolate the soil fungi (Kamal \& Bhargava 1971). This is a simple plating technique in which a small amount of soil is spread over a culture medium (Warcup 1950).

Materials required $-70 \%$ alcohol, alcohol lamp, dissecting microscope, lighter, fine forceps, cork borer, tissue papers, wrapper, PDA media plates

Methodology $-0.005 \mathrm{~g}$ of soil is spread in a sterilized Petri-dish and $10-15 \mathrm{~mL}$ of agar media at $35-40^{\circ} \mathrm{C}$ is poured onto the spread soil. Petri-dishes are rotated gently for fine dispersal before agar solidifies. If the soil is dry, few sterilized water drops are added into the plate before adding the media (Warcup 1950). Plates are incubated at an appropriate temperature for 1 week. After colonies appear, randomly selected fungal colonies are transferred separately for sub-culturing.

\section{Immersion tube method}

The immersion tube method was introduced to isolate a wide diversity of microflora from soil (Chesters 1940, 1948). A modified version of the above technique is as below.

Materials required $-70 \%$ alcohol, alcohol lamp, dissecting microscope, lighter, needle, aluminum foil, screw cap micro tube, fine forceps, cork borer, tissue papers, wrapper, PDA media plates

Methodology - Spirally punched, tiny holes are prepared on the walls of sterile screw cap micro-tubes using a red-hot needle. Tubes are sterilized and $1 \mathrm{~mL}$ of PDA is poured into the tube in laminar airflow. When the medium cooled down, the tubes are wrapped well using sterile aluminum foil and stored in a sterile tube until use. Collection sites are selected and soil layers below the litter layer are exposed using a flame sterilized cork borer. Screwcap micro-tubes are immersed at the required depth. Micro-tubes are incubated inside the soil for a week to provide adequate time for mycelia to grow in a nutrient medium. Tubes are transferred to sterile Petridishes and brought to the laboratory for analyses (Fig. 29).

\section{Isolating techniques from air}

Atmospheric air contains numerous fungal spores (Lacey \& Dutkiewicz 1994). There are two methods generally used for sampling atmospheric fungi as (i) Petri-dish gravitational or settle plate method (Pelczar et al. 1993) and (ii) Volumetric spore sampling method (Martinez-Ordaz et al. 2002). Evaluation of atmospheric fungi is important as these spores may cause respiratory diseases when inhaled by humans. Some of these spores also cause allergies (Şakıyan \& İnceoğlu 2003). Besides qualitative, different spore counts can be obtained by these gravitational and volumetric sampling methods. However, it is difficult to efficiently collect some small-spored fungi using the gravimetric method (O'Connor et al. 2014). 


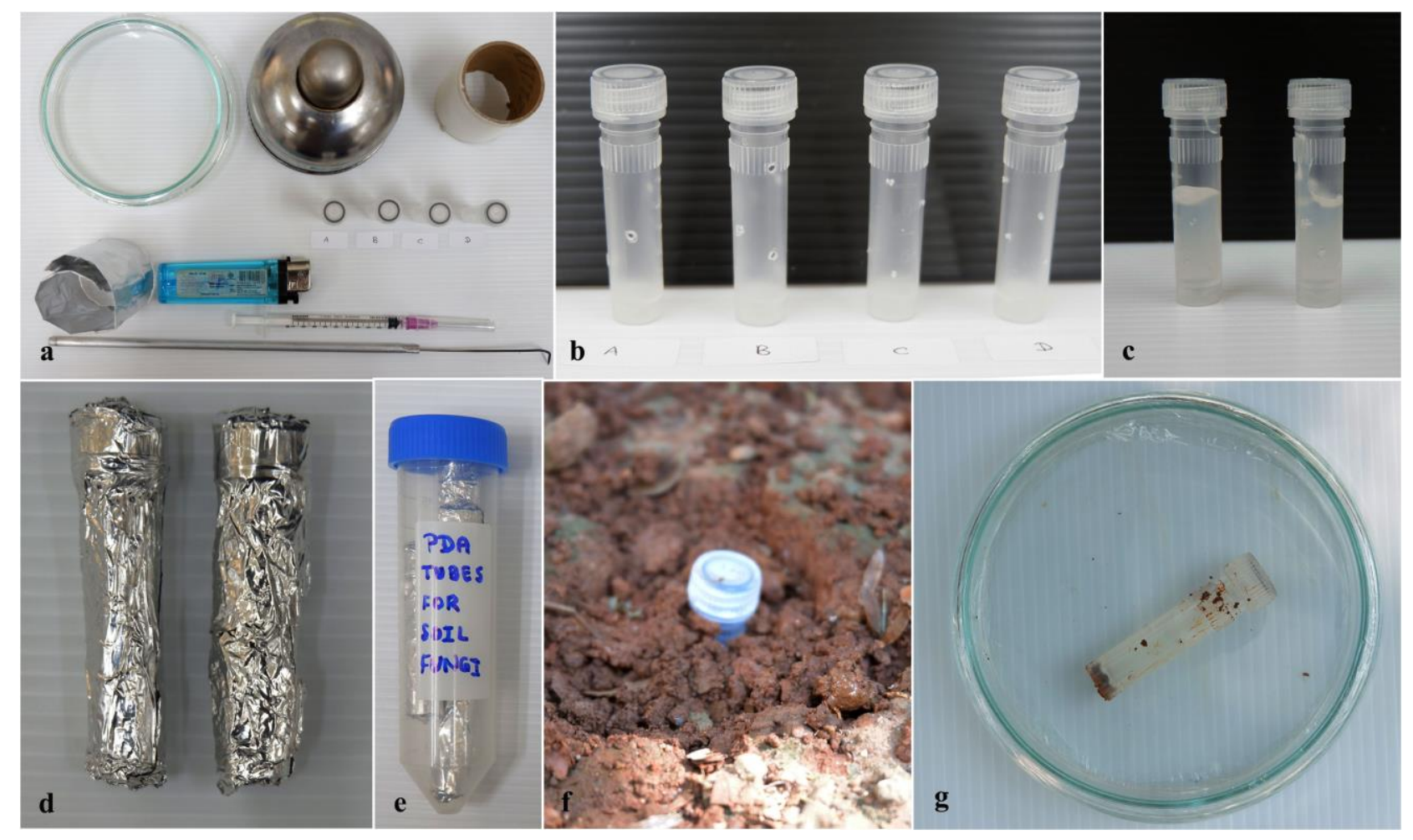

Figure 29 - Immersion tube method. a Tools. b Screw cap micro tubes with tiny holes. c Screw cap micro tubes with PDA medium. $d$ Aluminum foil-wrapped tubes. e Sterile tubes. $f$ Tubes immersed inside the soil. g Tube transferred to sterile Petri-dish.

\section{Petri-dish gravitational or settle plate method}

The settle plate method is a basic, passive sampling method for airborne fungi. A Petri-dish containing suitable agar medium is exposed to the atmosphere wherein the medium collects fungi that are settled by gravity (Napoli et al. 2012). There is however a greater possibility to collect large-spored fungi than small-spored ones, because of air turbulence around the plate can affect the results and small spores may lose and only occasionally settle (Scaltriti et al. 2007). The effect of air turbulence may minimize the spore settlement when the plate left out longer. Settle plates are also impossible to validate because there is no way to measure the volume of air sampled (Pasquarella et al. 2008).

Materials required - Petri-dishes with PDA, double-sided tape, tissue, wrapping tape, labels

Methodology - PDA plates are arranged horizontally and vertically, facing different directions. Exposed PDA plates are collected after 3 hours and incubated at $28^{\circ} \mathrm{C}$ for $5-7$ days to recover the fungal colonies (Fig. 30).

\section{Volumetric spore sampling method}

Active sampling is performed using a surface air sampler and the airflow rate is regulated between 10-180 L/min (Gregory 1954, Brook 1959). The advantage of the machine is that spores from a comparatively large area are concentrated on a small area (Napoli et al. 2012).

Materials required - Petri-dish with PDA, double-sided tape, tissue, wrapping tape, labels, air system sampler

Methodology - The sampler is placed beside the agar plates and the fungal spores sucked from air are spread on agar plates. After a particular period, plates are closed and incubated at a suitable temperature.

\section{Incubation, obtaining pure cultures and sub-culturing}

Incubation promotes the growth of cultures under appropriate conditions and is affected by nutrients in the medium, $\mathrm{pH}$, temperature and light (Meletiadis et al. 2001). It is important to 
provide suitable conditions to yield optimal growth, the viability of mycelia however, depends on the growth rate, culture media and method of storage (Sun \& Guo 2012). Therefore, it is necessary to perform periodic sub-culturing at reasonable intervals to maintain the viability and active growth of cultures. Fresh medium plates are used for sub-culturing and a small amount of mycelial tips are inoculated to media plates. Inoculated plates are incubated under suitable temperature and light conditions (Cai et al. 2009).

However, the original culture characteristics may change during periodic sub-culturing. Colonies of slow growing fungi become thin with less mycelium, through form matted or fluffy colonies in initial isolations ( $\mathrm{Su}$ et al. 2012). Initial fluffy mycelia may become sticky, slimy colonies on repeated sub-culturing. Some cultures form chlamydospores during colony maturation, which appear in brown (Hyde et al. 2010).

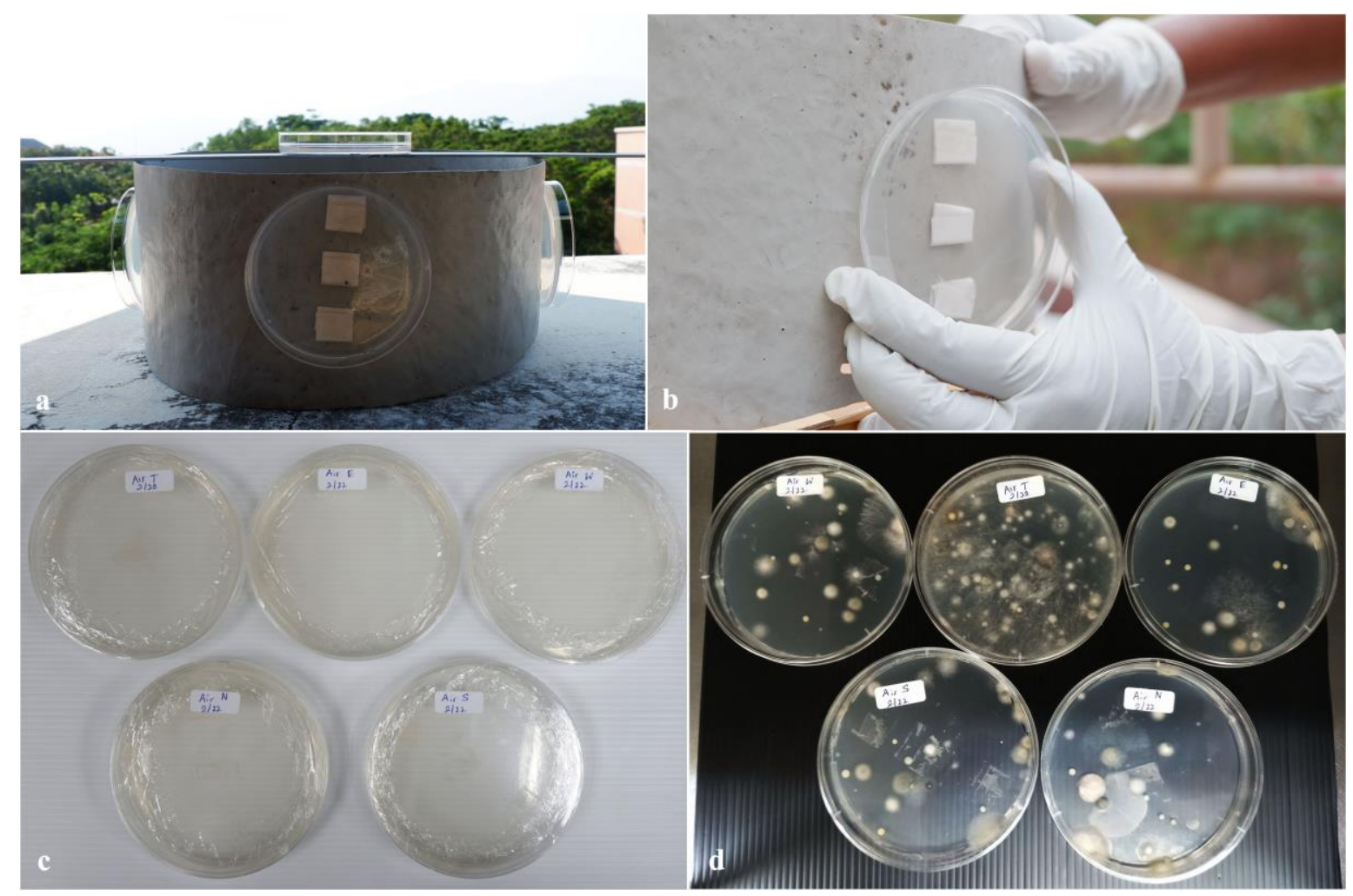

Figure 30 - The gravitation method (open plate technique). a Experimental setup. b Removing PDA plates. c Collected PDA plates. d Colony grown on PDA (after 3 days).

\section{Preservation and deposition at culture collections}

Fungi sometimes exist in hostile climatic conditions where most life cannot survive. Therefore, fungi are considered as one of the most abundant and diversified living organisms on earth (Whitman et al. 1998). However, only a small fraction of those fungi has been explored and studied. Thus, long-term preservation of fungal cultures is necessary to facilitate innovation derived from fungi and their activity.

An important and essential role of fungal culture collections is to make these fungal isolates available to the research community (Hawksworth 1985). Once a species is isolated from their natural habitat, it is useful as they are maintained for conservation and utilization (Humber 1997). Some fungi are host-specific and difficult to obtain culture. Growing ability of some fungi is reduced during sub-culturing due to unavailability of suitable conditions for preservation as well as lack of knowledge regarding conservation (Sharma et al. 2017). There are numerous fungal culture collections worldwide (Hawksworth 1985). Different culture collections have different rules for deposition and preservation. Therefore, there is a need to familiarize with the terms and conditions of culture collections before deposition. 
Ideally, the depositor prepares two screw valves or centrifuge tubes with agar slants and inoculates them with the pure culture (Hawksworth 1985). The inoculated tubes are stored at room temperature for a few days until the test fungus grows on the agar slant. After the cultures are grown on the agar slant, they are deposited with the culture data sheet, which contains the collection and isolation details, storage conditions and colony characters. Depositors may have to sign an agreement with the culture collection (Smith \& Onions 1994). Cultures are stored in suitable conditions to avoid frequent sub-culturing. Storage in mineral oil and demineralized, sterile distilled water only are elaborated as this can easily be carried out in every laboratory. Storage methods are complex and differ between culture collection and they include storage in mineral oils or demineralized, sterile water, lyophilization, storage at $-70^{\circ} \mathrm{C}$ and cryopreservation.

Sterilized mineral oil (liquid paraffin, medical paraffin) is used as a storage medium. Generally, $0.5 \mathrm{~cm}$ diam. mycelial discs are suspended in 1-2 $\mathrm{mL}$ of sterilized mineral oil (Kudriavtsev et al. 1972). Cultures on agar slants can also be covered with mineral oils to retain hydration, as this slows down metabolic activity and growth by reducing oxygen tension (Little \& Gordon 1967). Mineral oils prevent oxygen exchange between mycelial cells in the container and atmosphere, reduce metabolism and prevent desiccation of agar medium (Bunse \& Steigleder 1991). In addition, fungal cultures preserved in mineral oils are stored at $4^{\circ} \mathrm{C}$ for effective preservation. Retrieval of cultures is achieved by draining off excess oil and inoculating a small piece of the fungal colony on an agar medium with a sterile needle. However, it is better to wash the mycelium with sterilized water to remove the oil (Little \& Gordon 1967). The first subculture often shows a low growth rate. Cultures stored in mineral oil remain viable for 4-5 years. The disadvantages of this method are contamination by airborne spores and retarded growth upon retrieval (Sathe \& Dighe 1987). Storage in mineral oil is considered as a low-cost method with moderate genetic stability.

Storage in demineralized, sterilized water is an inexpensive method used to preserve the cultures for 2-5 years. Small mycelia discs of $0.5 \mathrm{~cm}$ are aseptically transferred to sterilized, precooled water and lids are tightly screwed. Culture tubes are stored at room temperature or at $3-5^{\circ} \mathrm{C}$ and preserved hyphae in water do not grow further due to lack of nutrients and oxygen (Bueno \& Gallardo 1998, Diogo et al. 2005). This method has successfully been used to preserve species in Mucoromycota and Kickxellomycota (Diogo et al. 2005).

\section{Avoiding contamination}

Fungal cultures often become an excellent source of food for mites when stored. Mites generally originate from fresh specimens and contaminate the culture plates through subculture and stacking plates together (Fig. 31). There are several guidelines to avoid mites in cultures.

1. The bench and stage of the microscope where the culturing is carried out is thoroughly cleaned with $70 \%$ ethanol.

2. All stored cultures are sealed with parafilm, although this does not keep out mites indefinitely.

3. All tools used to remove fungi from substrates are sterilized by $70 \%$ ethanol. The storage area and cabinets are kept clean and mite-free. This is achieved by sterilizing them with $70 \%$ ethanol as often as possible. If there are mite-contaminated plates in the collection, these are removed and decontaminated immediately. Cultures are stored in batches in separate sealed plastic bags.

4. Naphthalene pellets are put with cultures and this slows down the activity of mites. However, naphthalene may also reduce the growth of fungi.

5. Ivermectin, a chemical that inhibits insect growth and therefore, can be added to culture medium before pouring.

6. Culture containers can be immersed in jars with paraffin oil or water so that mites do not climb into the container.

7. Leaving plates in the incubator for a long time period may cause mite contamination. Therefore, all cultures should be transferred to storage vessels as soon as possible.

8. Mite proof Petri-dishes can also be used (e.g. $60 \mathrm{~mm}$ air-tight Petri-dishes), though they are 
expensive.

In addition to mites, bacteria are also the major contaminants of fungal cultures. Oh et al. (2018) have shown that 16 bacterial species isolated from fruiting bodies significantly influenced mycelial growth of filamentous fungi and molds. Most bacteria negatively affect filamentous fungal growth by inhibiting various enzymatic activities and using fruiting bodies as a nutrient source (Oh et al. 2018, Santos et al. 2003).

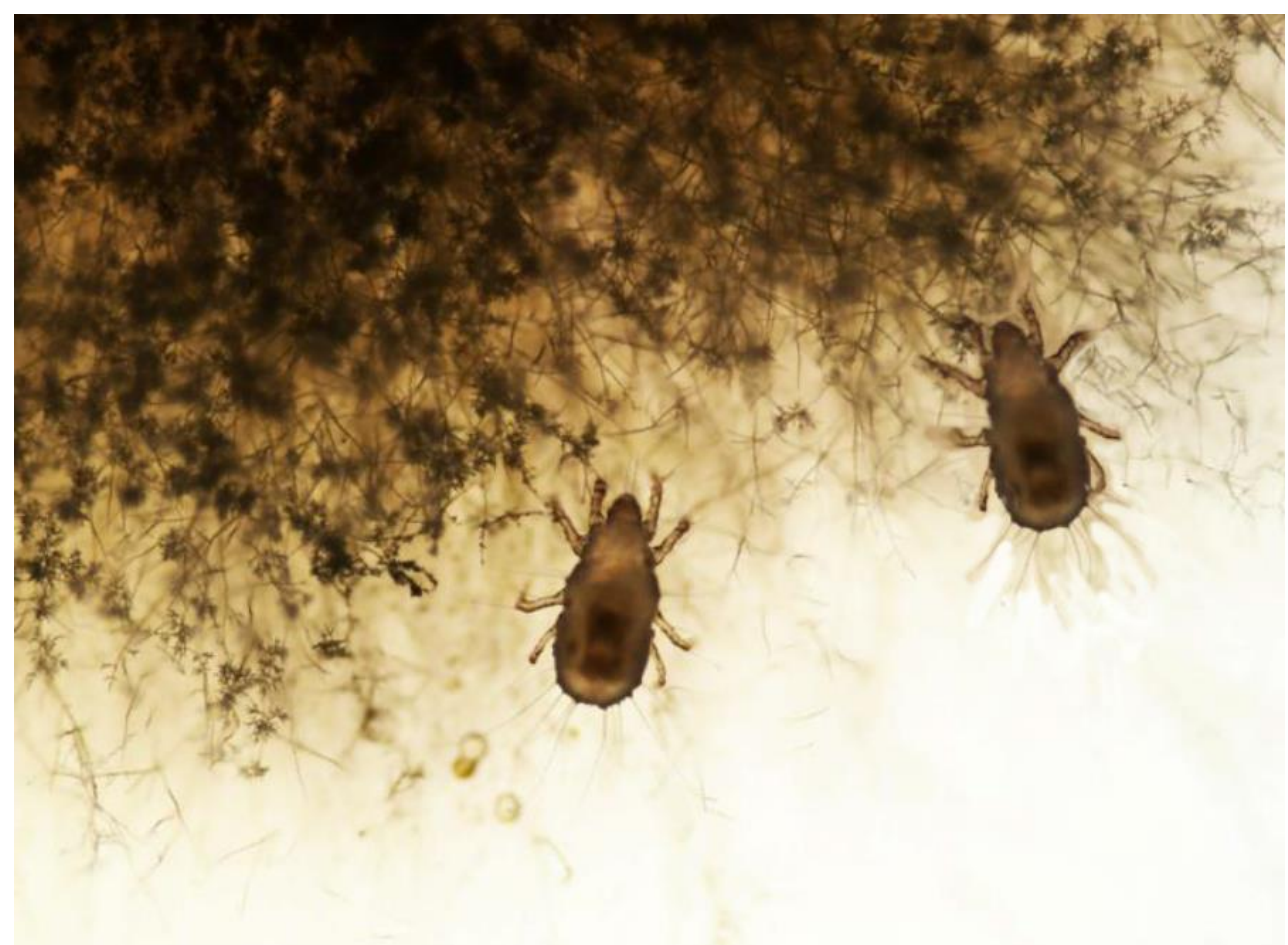

Figure 31 - Mites are eating fungal hyphae and laying eggs on cultures.

\section{Sporulation techniques}

Fungal sporulation or conidiation refers to the formation of spores or conidia from vegetative cells (Adams et al. 1998a) and it is influenced by environmental and endogenous biological rhythms (Wright 1979, Timberlake 1980, Su et al. 2012). Asexual sporulation may be an adaptive response that allows the organism to survive in undesirable conditions such as radiation, extreme heat or cold, and lack of nutrition. Spores are thick-walled, dormant structures that preserve the genetic content of the organism. Many experiments have been conducted to understand the molecular mechanisms of conidial sporulation (Roncal \& Ugalde 2003, Sun et al. 2012) because fungal spore and conidial morphology is very important in fungal taxonomy (Kirk et al. 2008).

Nutrient levels, host substrate and light are considered as major environmental factors that affect sporulation (Smith \& Berry 1974, Guo \& Michaelides 1998, Su et al. 2012). Different conditions and techniques have been used to facilitate fungal sporulation in commercial and scientific research (Xu et al. 2012). These techniques are pre-incubation in the dark, low temperature treatment, brushing mycelia and exposing mycelia to UV light (Guo et al. 2003, Wang et al. 2005, Xu et al. 2012). Other factors affecting fungal sporulation are mutilation of the mycelium (Rands 1917, Su et al. 2012), temperature (Prasad et al. 1973), pH (Yazdany \& Lashkari 1975) and humidity adjustments (Paul \& Munkvold 2005). Kashket \& Cao (1995) have reported that some fungi lose their sporulation ability during repeated transfer in artificial media.

\section{UV exposure}

Near exposure and direct exposure of mycelia to ultraviolet (UV) radiations for a short time can successfully induce conidial sporulation (Marsh et al. 1959, Dahlberg \& Etten 1982, Dhingra \& 
Sinclair 1985, Betina 1995, Xu et al. 2009, Su et al. 2012). Mycelia are exposed to UV light radiations with the wavelength range of $350-500 \mathrm{~nm}$ for 12 hours and darkness for 12 hours to imitate diurnal cycles of light and darkness (Marsh et al. 1959, Leach 1962, Dahlberg \& Etten 1982, Crous et al. 2006, Su et al. 2012). Light radiation-sensitive fungi can sporulate by this method. However, light radiation may hinder the sporulation of nocturnal sporulators such as Alternaria, Choanephora, Helminthosporium, Peronospora and Stemphylium (Dhingra \& Sinclair 1985). In addition, 18 to 24 hours of exposure to strong fluorescent or blue light has successfully induced sporulation in some species of ascomycetes (Crous et al. 2006).

\section{Low pH}

The fungal vegetative phase grows well in neutral to alkaline media and an increase in acidity may enhance the sporulation (Tatiana et al. 2010).

\section{Aeration}

Excess ammonia and carbon dioxide act as inhibitors of sporulation in some ascomycetes. Culture plates and cotton-plugged tubes with sufficient gas exchange can prevent the accumulation of $\mathrm{NH}_{3}$ and $\mathrm{CO}_{2}$ during fungal metabolic activities (Dhingra \& Sinclair 1985).

\section{Growing on nutrients depleted media}

Carbon, nitrogen and other microelements are the major nutrient factors in effective growth of fungal cultures (Timnick et al. 1951) and there are varieties of culture media used in fungal isolation and culturing (Booth 1971, Su et al. 2012). Even though these media provide promising growth for most saprobic, endophytic and pathogenic microfungi, their use has not induced sporulation of sterile isolates (Guo \& Michaelides 1998, Li et al. 2007, Su et al. 2012). Most fungi have specific carbon and nitrogen requirements for sporulation (Gao et al. 2007). Sporulation is less promising when mycelium growth is rapid and good culture media for sporulation generally leads to insipid growth of vegetative hyphae (Dahlberg \& Etten 1982, Dhingra \& Sinclair 1985, Nebane \& Ekpo 1992, Elson et al. 1998, Braun et al. 2011). Synthetic nutrient-poor agar, water agar (WA), $1 / 2$ or $1 / 4$ strength PDA are used as typical low nutrient media (Nirenberg 1976, Masangkay et al. 2000, Su et al. 2012). However, some fungi have specific requirements of carbon and nitrogen for sporulation when grown in nutrient-poor media (Dahlberg \& Etten 1982, Su et al. 2012). A possible mechanism for stimulation of sporulation pertains to sending wrong signals to the cells about environmental nutrient status (Adams et al. 1998b). Some Mucor species have records showing sporulation in synthetic mucor agar, while members in Kickxellomycetes sporulate on half-strength malt extract-yeast extract agar or cornmeal agar (Kurihara et al. 2004, Swathi-Sri \& Subrahmanyam 2017).

\section{Two stage cultivation}

In this method, one half of the agar plate is inoculated by the fungi required to sporulate and another half of the plate is inoculated by the fungus only performing vegetative growth. This forms competition for nutrients between both fungi (Sun et al. 2009).

\section{Low temperature}

Many researchers have confirmed that room temperature is suitable for sporulation for most fungi, while others have suggested that low temperature also works well in inducing sporulation (Shahin \& Shepard 1979, Tatiana et al. 2010).

\section{Chemically induced sporulation}

Some chemicals such as $\mathrm{CaCO}_{3}$ may increase sporulation in some Alternaria species (Shahin \& Shepard 1979, Masangkay et al. 2000, Su et al. 2012). Xu et al. (2009) has proposed a novel method to promote the conidial formation of Pochonia chlamydosporia, using hydrogen peroxide together with two-stage cultivation which enhanced sporulation faster than the regular continuous 
cultivation. Fungal colonies grown on PDA are briefly treated with $90 \mathrm{mM}$ concentrations of $\mathrm{H}_{2} \mathrm{O}_{2}$, followed by fungal plugs transferred into water agar (WA) for sporulation (Xu et al. 2009).

\section{Host tissue}

The addition of host tissues on vegetative mycelia is often used to induce sporulation in host specific and plant pathogenic fungi (Gao et al. 2007, Su et al. 2012). Dhingra \& Sinclair (1985) showed that host substrates such as leaves, fruits, roots, straw and stems which are sterilized by propylene oxide, autoclaved twice at $121^{\circ} \mathrm{C}$ for 20 minutes, or with $75 \%$ alcohol (Crous et al. 2006) facilitated fungal sporulation. $1.5 \%$ water agar is poured into a sterile Petri-dish which contains sterilized host substrates. After the medium solidifies, Petri-dishes are moved to the propylene oxide chamber for further sterilization. Petri-dishes are then inoculated with fungal mycelia and incubated until sporulation (Yoshida \& Shirata 2000). Several studies have shown that providing a host substrate on which the taxon originally occurred are successfully induced the sporulation. It is important to use healthy, recently picked, host tissues (Crous et al. 2006). Alternaria, Colletotrichum, Curvularia, Drechslera, Fusarium, Helminthosporium, Nigrospora, Pestalotia, Phaeotrichoconis, Plenodomus, Pyricularia, Trichoconis and Botryosphaeriaceae species successfully sporulated in $2 \%$ water agar with sterilized pine needles incubated under closely exposed UV light radiation (Crous et al. 2006, Wulandari et al. 2009, Glienke et al. 2011). Addition of invertebrate-derived substrate to the media was proven successful for Coemansia asiatica, Coemansia javaensis, Linderina macrospora and species of Kickxellomycota (Kurihara et al. 2008). However, this substrate did not consistently yield positive results.

\section{Dehydration-mycelial injury method}

This method has been used successfully to induce sporulation in Alternaria solani, Drechslera poae, Cochiobolus sativus and Pyrenophora dictyoides (Dhingra \& Sinclair 1985). The fungus is grown in a shaking flask for sufficient mycelium growth. The mycelium suspension is then blended for two minutes to wound the hyphal wall, followed by centrifugation to concentrate and harvest the mycelium. The pellet is re-suspended in $0.02 \mathrm{M}$ phosphate buffer $(\mathrm{pH}$ 6.4). Then two $\mathrm{mL}$ of the suspension is distributed onto sterile, dry filter paper placed on the culture plate. Small agar blocks are placed on filter paper in media and incubated at room temperature. The hyphae grown on agar blocks are sporulated and the spores can be washed using a wash bottle. Placing sterile filter paper, lens cleaning paper or blotting papers on the agar surface dehydrates the mycelia and induces sporulation in many fungi. Dehydration prevents bacterial growth on cultures (Paul \& Munkvold 2005).

\section{Fungaria: preparation, conservation and handling}

Fungarium specimens are a vital source of information of all taxa and provide a permanent, verifiable record of the occurrence of a taxon at a particular time and place (Hawksworth 2010, Seifert \& Rossman 2010). A fungarium specimen is a dried material, dried substrate of its host, dried culture or microscope slide (Fig. 33) deposited in fungaria (Fig. 32). Fungi are collected from a range of organic or inorganic substrates such as any part of plants, humans, animals, and mushrooms (Blackwell 2011, Hyde et al. 2019). Inanimate objects such as clothes, concrete, rocks, marine sediments, metals, plastic materials, sand and glass can be the substrates as well.

Fungarium specimens are very important in all aspects of fungal systematics and appropriate preparation, storage and careful handling of specimens to ensure the good condition to use in future research (Hosoya et al. 2017). They can also be used for DNA extraction (Smith et al. 2020, Sugita et al. 2020). Morphology of a taxon can be referred from fungarium specimens (Andrew et al. 2019). If the morphological descriptions are poor, it can be improved by examining the fungarium specimens (Guarro et al. 1999). In addition, they provide information on the biogeographical distribution of taxa. 


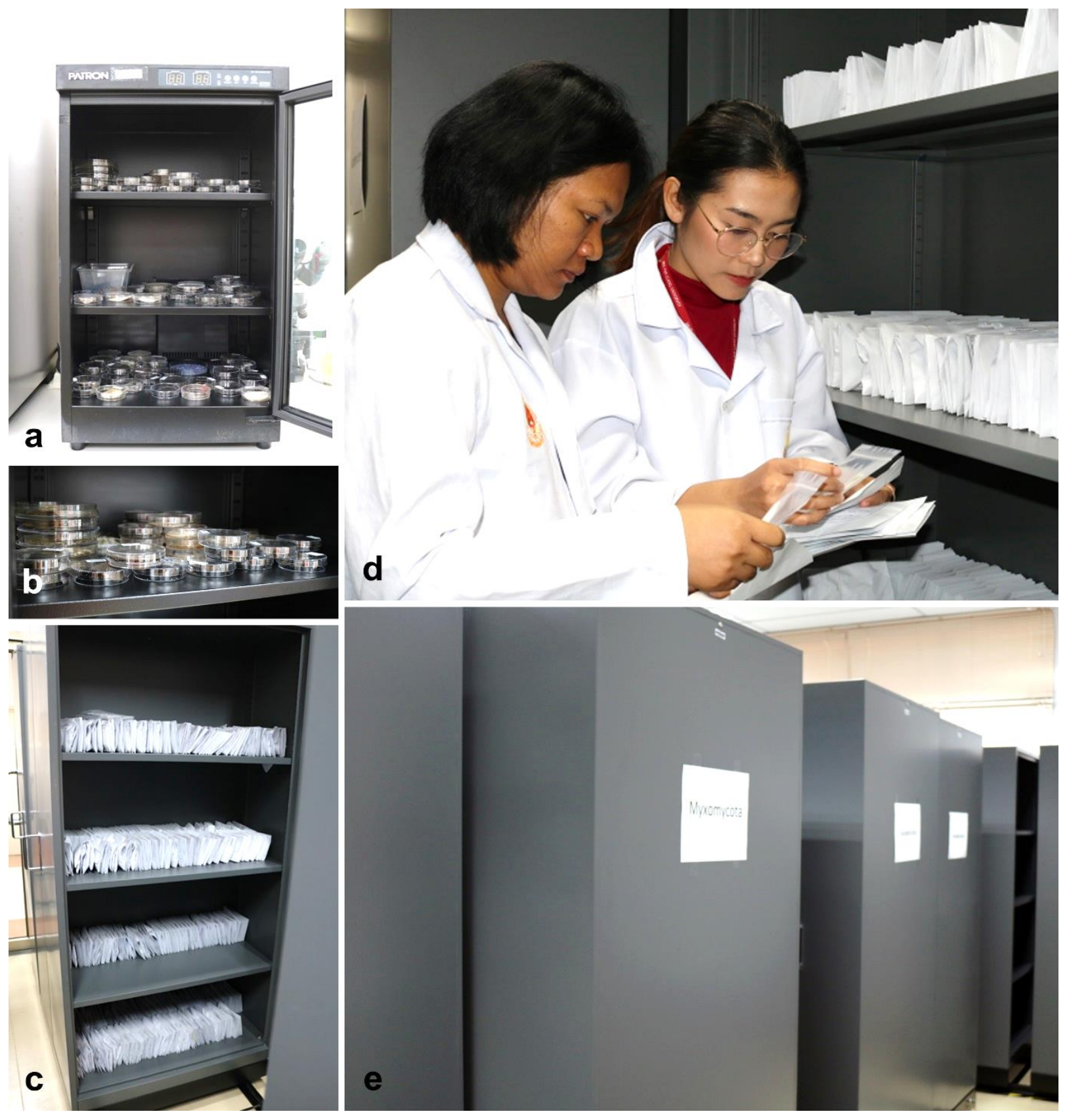

Figure 32 - Storage of specimens in a fungarium. a, b Drying oven for fungal cultures. c-d Cabinets with properly labeled specimens. e Storage room with controlled temperature and humidity.

\section{Fungaria specimen preparation}

Studies on fungal pathology, diversity and ecology are revealing many new species (Hyde et al. 2018). The host or material on which the species occurs serves as an important criterion to recognize a fungus (Dayarathne et al. 2016). The holotypes, isotypes, paratypes, and lectotypes represent different species, genera and families (Senanayake et al. 2018). Not only are the types useful in understanding the historical context of a fungus, but also important in future education and training (Thiers et al. 2013). When fungal classification changes and species taxonomy are shifting based on new knowledge, voucher specimens help to cross-reference these changes with previous research. Therefore, the preservation of specimens is essential. Well-prepared voucher specimens, cultures or slides should be deposited in recognized, accessible fungaria or institutions and cited in the publication to ensure scientific reliability of published accounts (Robert et al. 2013, Andrew et al. 2019). 


\section{Dried material}

Fresh fungal specimens are air-dried or oven-dried as soon as possible once photographs have been taken. If it is impossible to dry the specimens as soon as collected, they are stored in a refrigerator, but not the freezer (Andrew et al. 2019). Specimens are dried using hot air or 60-100watt desk lamp. Temperature between $40-45^{\circ} \mathrm{C}$ for $6-12$ hours is ideal for most specimens.Specimens are dried at $25-35^{\circ} \mathrm{C}$ for $2-3$ days when the material is thick and the fruiting bodies are cracked or shrunk dramatically losing their moisture, causing discolouration and changes in shape (Antonovics et al. 2003, Willis et al. 2017). Drying avoids mold growth and pest contamination.

The packaging depends on the size of the specimen, but should ideally follow the rules and regulations of the fungaria where the material is deposited (Andrew et al. 2019). Thin specimens such as leaves can be stored in envelopes, while specimens such as seeds, stems, twigs or macrofungi can be stored in hard paper boxes. A suitable glue, liquid latex or double-sided adhesive tapes are used to stick the labels. Three-dimensional specimens can be fixed with board pins or placed in grooves or slits on the supporting material (Molin et al. 2011).

\section{Permanent microscope slides}

Permanent microscope slides that show morphological characters of the taxon can be a fungarium specimen (Mueller et al. 2004a). These are very useful where the specimen has a limited amount of the fungus or has unique characteristics. The details of fungal mounting techniques and slide preservation are explained under mounting.

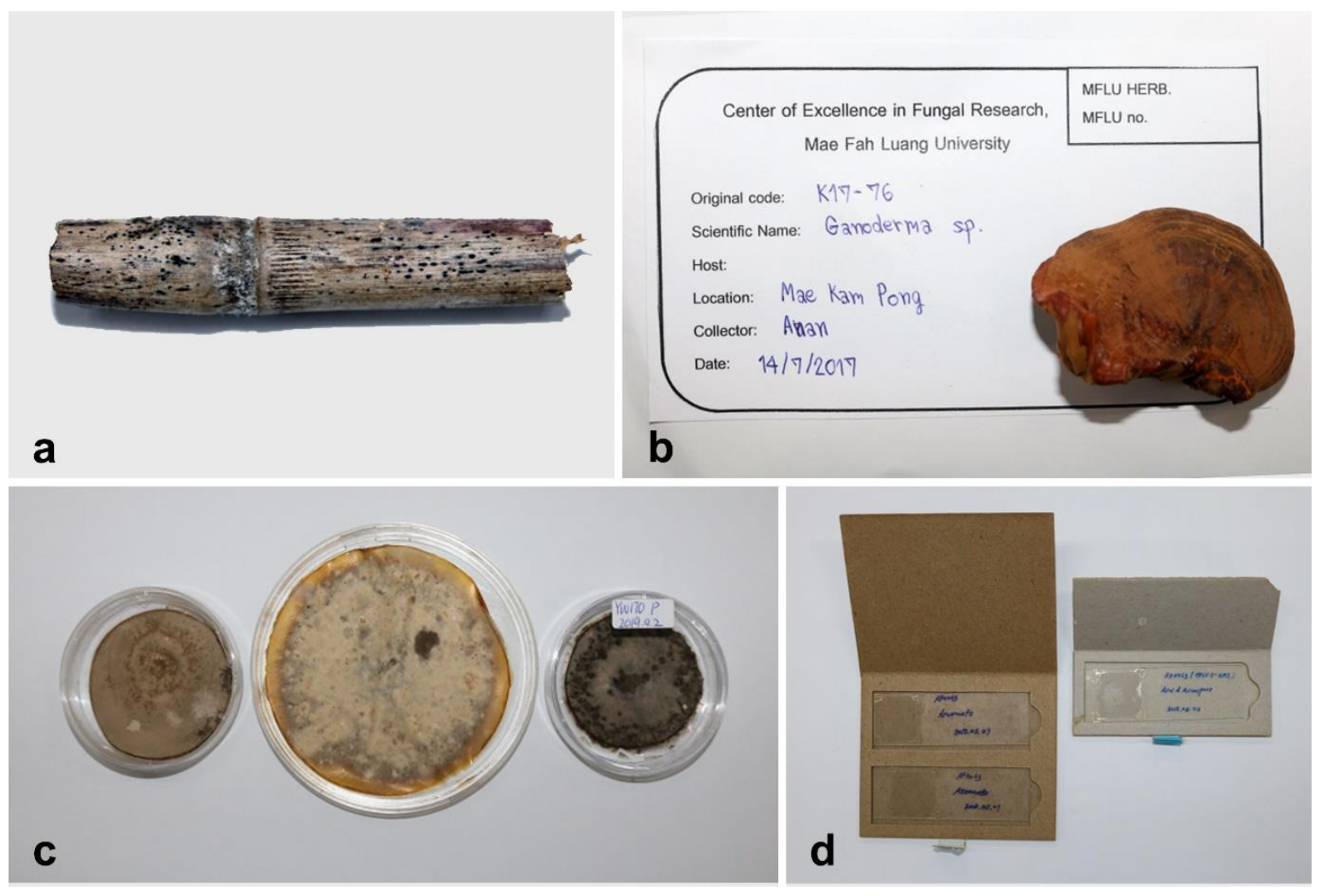

Figure 33 - Fungarium specimens. a Dried woody material. b Dried mushroom. c Dried culture. d Permanent microscpe slide.

\section{Dried cultures}

Fungal cultures not only preserve morphological characters of hyphae or colonies but also reproductive structures (Andrew et al. 2019). Therefore, dried cultures should be deposited as part of a fungarium specimen. Taxa discovered from environmental samples may only be a culture (Onions 1971). Cultures grown in water agar with $2.5 \%$ glycerol are examined for fruiting 
structures and other morphological characters. The base of an aluminum tray is covered with silica gel and dried in a hot-air oven at $160^{\circ} \mathrm{C}$ for 2 hours and let it cool inside the oven (Rogers 1914). Culture plates are placed on the aluminum tray with silica gel. The tray with cultures is placed in a desiccator. Cultures are continuously observed for drying and taken out before shrinking. Dried cultures are stored in wax paper bags with silica gel (Silva et al. 2011).

\section{Care of fungarium specimens}

Fungarium specimens ideally preserve all morphological characters of a fungus. Specimens are arranged on the hardboard to show the characters well and this prevents shrinking during drying. The specimens are placed on mounting boards and are stuck to the boards using glue. They are stored in suitable packaging with a tag (Wu et al. 2004). Generally, fungarium specimens are stored at humidity between $40-45 \%$ and temperatures lower than $20^{\circ} \mathrm{C}$ (Rogers 1914 , Andrew et al. 2019). If specimens are collected from the decaying litter or soil, then insects and their eggs are removed first. Dried specimens are placed in zip-lock bags and frozen at $-20^{\circ} \mathrm{C}$ for seven days (Rogers 1914). Insects and their larva are destroyed during freezing. Specimens are then taken out and kept at room temperature overnight allowing any remaining eggs of insects to hatch. The specimens are frozen again for another seven days (Wu et al. 2004).

Labeling of fungarium specimens is very important. Each specimen gets a unique fungarium reference number (Meineke et al. 2018). Generally, important data that should be labeled with the specimen are fungus name, locality, host, substrate, collector's name, collected date, temporary code, type status and collection code of the specimen (Dentinger et al. 2016). The collector's number is written on tags that can be attached to the specimen and its duplicates (Mueller \& Schmit 2007).

Locality refers to the exact geological position of the specimen collection and the closest reference points are included when recording the locality such as the nearest town or a well-known landmark along with the exact locality. This information can be obtained from maps together with a GPS (Global Positioning System) reading (Bruns 2012). The collected date and location provide information about the life cycle and distribution of the desired fungus. This can be used when planning subsequent trips to recollect the specimens or to collect the fungi in similar other areas (Thiers \& Halling 2018).

Specimens can often be destroyed by mites. Therefore, fumigation is performed once a year using suitable fumigates such as methyl bromide (De Gasper et al. 2014). They are handled with care to make sure they are not crushed or deteriorated due to poor fungarium storage conditions. Broken packaging or covers cause misplacing or damaging of specimens by pressure, moisture, pests, chemicals and fire (Bruns 2012, Thiers et al. 2013). Specimens are damaged due to the usage of unsuitable, non-archival materials, overuse of stains or splashes of stains and chemicals, surface dirt, insect damages and wasting or overusing of the specimen (Dentinger et al. 2016, Thiers \& Halling 2018). In addition to the physical damages, taxonomical losses can occur in old specimens such as lack of enough fruiting bodies on specimens due to examination by mycologists and loss of some morphological characters (Senanayake et al. 2018).

\section{Documents need to attach with the specimens when posting}

Fungarium specimens are biological materials and can only be sent out or loaned for scientific studies. All rules and regulations according to international and regional laws should be followed (Thiers 2020). At all times, fungarium materials loans should have the following documents.

\section{Material Transfer Agreement (MTA)}

The material transfer agreement is a document signed between the party that sends the specimen and the party that receives the specimens to explain their rules and regulations (Verkley et al. 2020). There should be a specific label on the packaging to clarify that the specimen contained is a 
"dry fungarium specimen for scientific study with no commercial value" and any invoice should also be included.

2. Some other useful documents depending on the fungarium institute and country.

Most countries have restricted sending biological materials across the borders post COVID 19 pandemic. Some countries may quarantine the packages for 14 days especially if packages are from high risk areas.

3. Phytosanitary certificate.

Plant quarantine procedures are needed when transporting fungi with plant parts across the borders of some countries (Laird 2002).

4. Permit to import laboratory specimens (e.g: one year permit)

\section{Recommendation for the numbers of cultures, fungarium specimens and sequence data needed for publication}

Preserved cultures in culture collections are metabolically inactive (Seifert \& Rossman 2010). This can be an ex-type strain, authentic strain, original strain or reference strain. Ex-type living cultures should be deposited when introducing a new species (Agerer et al. 2000, Senanayake et al. $2017 \mathrm{~b}$ ) while cultures obtained from new hosts and geographical records or specimens of extant species can be reference strains (Doilom et al. 2017). All published cultures are deposited in an accessible culture collection. Hence, we provide the following recommendations:

1. Ex-type strains should preferably be deposited in at least two easily accessible culture collections and it will ensure that cultures are available in at least one collection for future studies. Cultures can be contaminated or lost. Thus, depositing more than one culture collections will ensure the safeguarding of the deposited cultures.

2. Ex-type strains should also be kept in a personal culture collection with replicates. Personal culture collections are quickly accessible resources and researchers can obtain any additional data easily from cultures such as sequence data, colony and phenotypic characters.

3. Three isolates should preferably be obtained from the same specimens, but from different fruiting bodies to compare their colony characters.

4. Isolates obtained from a holotype, isotype and paratype should preferably be sequenced and DNA sequences deposited in GenBank.

5. Nontype strains are also very important to confirm the taxonomic characters of the desired taxon. If there are some missing characters or details in the original description such as asexual or sexual morph, and colony characters on different media of the taxon can be obtained from reference strains, this may add additional value to the taxon. Reference strains can be used to confirm existing characters of those taxa too.

6. Colony characters should be obtained from the original cultures as these characters are subjected to changes after several sub-culturing.

7. The holotypes are generally deposited at the nearest accessible repository to the collected locality or accessible repositories where authors are affiliated. Isotypes or paratypes should be deposited in different fungaria if available and this ensures the availability of the specimens for future studies (Seifert \& Rossman 2010).

\section{Discussion}

Numerous fungal and fungus-like species have been discovered yearly over the last decades (Wu et al. 2019). This has resulted in an increased interest in fungal taxonomy and the development of new tools and methods in fungal systematics (Guarro et al. 1999). Phenotypic characters are the fundamental attributes in myco-taxonomy and it gives a basic figure of an unknown taxon. Morphology provides a particular dimension to the taxonomy of a species and nowadays these are supported with DNA sequence based phylogeny and its evolutionary relationships can be inferred (Hyde \& Soytong 2008). However, there is no general consensus among taxonomists about which morphological criteria should be emphasized on (Guarro et al. 1999) and different taxonomists 
adopted different methods. The techniques used in morphological studies are scattered in different papers and the main objective herein was to provide a synthesis and attempt to summarise widely used and ease mycologists task in the search of a particular method. The commonly used techniques for microfungi has been reviewed and discussed herein under five major topics.

Morphological examination and isolation techniques are important to obtain a taxonomic description of a taxon (Sun \& Guo 2012, Fernandes et al. 2015). The techniques or methodologies can greatly influence perceived fungal diversity and must constantly reduce the practical problems as far as possible (Hyde \& Soytong 2008). However, the selected techniques are based on the facilities in the laboratories, cost for the experiments and the availability of expertise.

Mycologists in developing countries use low-cost techniques (Sati 2006). The innovation of simple methods or methods which are low in cost is needed for developing countries. As an example, an aseptic workbench is better than laminar airflow when considering the cost. In addition, use of toxin free, low cost chemicals and reagents are encouraged to protect the environment because the toxic chemicals have harmful health effects.

In old Protologues, some morphological characters were either not examined or not included, while recent descriptions include more character details. Some important characters that were not originally examined are revealed in recent studies using modern microscopy and based on these newly investigated characters, previously described species may be introduced as new ones. Therefore, it is necessary to compare the same set of morphological characters of new collections with the previous collections of existing species that have poor Protologues.

Technology has played an important role in the expansion of taxonomy (Guarro et al. 1999. Specifications of microscopes have technologically advanced during the last several decades (Swedlow 2012). Therefore, accurate measurements and characters can be obtained. However, the measurements of a fresh specimen may not always totally tally with the measurements of holotype in the same species because of technical errors. Therefore, a combination of characters and their size, shape, colour, ecology, biological activities and molecular phylogeny are considered while delineating species (Lodge et al. 2014).

\section{Acknowledgements}

I.C. Senanayake thanks to Kevin D. Hyde, Putarak Chomnunti, Saranyaphat Boonmee, Nalin N. Wijayawardene, Mingkwan Doilom for his great work on this manuscript and also appreciate the kind support given by the laboratory staff of College of life science and Oceanography, Shenzhen University, Shenzhen, China and Center of Excellence in Fungal Research, Mae Fah Luang University, Chiang Rai, Thailand for preparation of illustrations and doing experiments. I.C. Senanayake thanks Chiang Mai University, Thailand. Our special thanks go to staff members of the Mae Fah Luang University Herbarium. K.D. Hyde would like to thank "Impact of climate change on fungal diversity and biogeography in the Greater Mekong Subregion" (RDG6130001). D.N. Wanasinghe would like to thank the CAS President's International Fellowship Initiative (PIFI) for funding his postdoctoral research (number 2019PC0008), the $64^{\text {th }}$ batch of China Postdoctoral Science Foundation (No Y913083271).

\section{References}

Abdulkhair WM, Alghuthaymi MA. 2016 - Plant Pathogens, Plant Growth, Everlon Cid Rigobelo, IntechOpen, Doi: 10.5772/65325

Adams HL, Thomas CR. 1988 - The use of image analyses for morphological measurements on filamentous microorganisms. Biotechnol Bioeng 32, 707-712.

Adams RM, Hurd BH, Lenhart S, Leary NA. 1998a - Effects of global climate change on agriculture: an interpretative review. Climate Res 11, 19-30.

Adams TH, Wieser JK, Yu JH. 1998b - Asexual sporulation in Aspergillus nidulans. Microbiol Mol Biol Rev. 62(1), 35-54. 
Adds J, Larkcom E, Miller R, Sutton R. 1999 - Tools, techniques and assessment in biology: A course guide for students and teachers. Nelson Thornes.

Adnan RM, Alshammari E, Ashraf SA, Patel K et al. 2018 - Physiological and Molecular Characterization of Biosurfactant Producing Endophytic Fungi Xylaria regalis from the Cones of Thuja plicata as a Potent Plant Growth Promoter with its Potential Application. BioMed Res Int 1-11.

Agerer R, Ammirati J, Blanz P, Courtecuisse R et al. 2000 - Always deposit vouchers. Mycol Res 104, 642-644.

Ahmad I, Ahmad Khan MS. 2012 - Microscopy in mycological research with especial reference to ultrastructures and biofilm studies. Current microscopy contributions to advances in science and technology (A. Méndez-Vilas, Ed.), Formatex Research Center, Spain, 646-659.

Ahmed M, Hussain M, Dhar MK, Kaul S. 2012 - Isolation of microbial endophytes from some ethno-medicinal plants of Jammu and Kashmir. J. Nat. Prod. Plant Resour 2 (2), 215-220.

Ainsworth GC. 1976 - Introduction to the history of mycology Cambridge University Press, Cambridge, UK.

Ali MM. 1962 - Comparison of the physiology of three isolates of Colletotrichum graminicola. Mycopathologia et Mycologia Applicata 17, 261-268.

Ali-Shtayeh MS, Jamous RMF. 2000 - Keratinophilic fungi and related dermatophytes in polluted soil and water habitats. Revista Iberoamericana de Micologia 17, 51-59.

Almeida D, Gusmao LFP. 2015 - Modification to the Barber and Keane's method of illustrating fungi. Mycosphere 6, 630-633.

Ampt EA, van Ruijven J, Raaijmakers JM, Termorshuizen AJ, Mommer L. 2019 - Linking ecology and plant pathology to unravel the importance of soil-borne fungal pathogens in species-rich grasslands. Eur J Plant Pathol 154, 141-156.

Andrew C, Diez J, James TY, Kauserud H. 2019 - Fungarium specimens: a largely untapped source in global change biology and beyond Philos. Trans R Soc Lond B Biol Sci 37420170392.

Andrews S, Pitt JI. 1986 - Selective medium for isolation of Fusarium species and dematiaceous hyphomycetes from cereals. Appl Environ Microbiol 51, 1235-8.

Anjum N, Chandra R. 2019 - Endophytic bacteria of Catharanthus roseus as an alternative source of vindoline and application of response surface methodology to enhance its production. Arch Biol Sci 71, 27-38.

Antonovics J, Hood ME, Thrall H, Abrams JY, Duthie GM. 2003 - Herbarium studies on the distribution of anther-smut fungus (Microbotryum violaceum) and Silene species (Caryophyllaceae) in the eastern United States. Am J Bot 90, 1522-1531.

Arai T, Mikami Y, Yokoyama K, Kawata T, Masuda K. 1977 - Morphological Changes in Yeasts as a Result of the Action of 5-Fluorocytosine. Antimicrobial agents and chemotherapy 12(2), 255-260.

Ariyawansa HA, Hawksworth DL, Hyde KD, Jones EBG et al. 2014 - Epitypification and neotypification: guidelines with appropriate and inappropriate examples. Fungal Divers 69, 57-91.

Arnold AE. 2007 - Understanding the diversity of foliar fungal endophytes: progress, challenges, and frontiers. Fungal Biol Rev 21, 51-66.

Ataides D, Pamphile J, Garcia A, Ribeiro M et al. 2018 - Curvularin produced by endophytic Cochliobolus sp. G2-20 isolated from Sapindus saponaria L. and evaluation of biological activity. J Appl Pharm Sci 8, 32-37.

Ayse-Dilek AZAZ. 2003 - Isolation and Identification of Soilborne Fungi in Fields Irrigated by GAP in Harran Plain Using Two Isolation Methods. Turk J Bot 27, 83-92.

Aziz NH, Zainol N. 2018 - Isolation and identification of soil fungi isolates from forest soil for flooded soil recovery. IOP Conf. Series: Mater Sci Eng 342: 012028.

Baldauf SL, Palmer JD. 1993 - Animals and fungi are each other's closest relatives: congruent evidence from multiple proteins. Proc Natl Acad Sci 90, 11558-62. 
Bamisile B, Dash CK, Akutse K, Qasim M et al. 2019 - Endophytic Beauveria bassiana in FoliarTreated Citrus limon Plants Acting as a Growth Suppressor to Three Successive Generations of Diaphorina citri Kuwayama (Hemiptera: Liviidae). Insects 10, 176.

Barber P, Keane P. 2007 - A novel method of illustrating microfungi. Fungal Divers 27, 1-10.

Barr DJS. 1986 - Allochytridium expandens rediscovered: Morphology, physiology and zoospore ultrastructure. Mycologia 78, 439-448.

Barr DJS. 1990 - Phylum Chytridiomycota. In: Margulis (ed.) Handbook of protoctista. Jones and Bartlett, Boston, 454-466.

Barr ME. 1978 - The Diaporthales in North America: with emphasis on Gnomonia and its segregates. Mycol Mem 7, 1-232.

Barr ME. 1987 - Prodromus to Class Loculoascomycetes. M.E. Barr Bigelow, Amherst, Massachusetts

Barron GL 1972 - The genera of Hyphomycetes from soil. Robert E. Krieger Publishing Co. Inc., New York.

Barry DJ, Williams GA. 2010 - Microscopic characterization of filamentous microbes: towards fully automated morphological quantification through image analyses. J Microsc 244, 1-20.

Bärtschi C, Berthier J, Guiguettaz C, Valla G. 1991 - A selective medium for the isolation and enumeration of Mucor species. Mycol Res 95, 373-374.

Basu S, Bose C, Ojha N, Das N et al. 2015 - Evolution of bacterial and fungal growth media. Bioinformation 11, 182-4.

Beneke ES, Rogers AL. 1980 - Medical Mycology Manual with Human Mycoses Monograph. Minnesota Burgess Publishing Company, Minneapolis.

Benny GL. 2008 - Methods used by Dr. R. K. Benjamin and other Mycologists to isolate Zygomycetes. Aliso 26, 37-61.

Bernadovičová S, Ivanová H. 2011 - Hyphomycetes and Coelomycetes fungi isolated from affected leaves and twigs of cherry laurel trees. Folia Oecologica, 38, 137-145.

Bernardi-Wenzel J, Garcia A, Filho C, Prioli A, Pamphile J. 2010 - Evaluation of foliar fungal endophyte diversity and colonization of medicinal plant Luehea divaricata (Martius et Zuccarini). Biol Res 43, 375-384.

Betina V. 1995 - Photoinduced conidiation in Trichoderma viride. Folia Microbiol 40, 219-224.

Bills GF, González-Menéndez V, Martín J, Platas G et al. 2012 - Hypoxylon pulicicidum sp. nov. (Ascomycota, Xylariales), a Pantropical Insecticide-Producing Endophyte. PLoS ONE 7, e46687.

Blackwell M. 2011 - The Fungi: 5.1 million species? American Journal of Botany 98, 426-438.

Blauda A, Menon M, van der Zaanb B, Lairc GJ, Banwarta S. 2016 - Effects of Dry and Wet Sieving of Soil on Identification and Interpretation of Microbial Community Composition. Advances in Agronomy 142, 119-142.

Bolton MD, Thomma BPHJ. 2012 - Plant Fungal Pathogens: Methods and Protocols. (Methods in Molecular Biology; No. 835). Humana Press, New York, USA.

Booth C. 1971 - Methods in Microbiology. Academic press INC LTD, London.

Bortolus A. 2008 - Error Cascades in the Biological Sciences: The Unwanted Consequences of Using Bad Taxonomy in Ecology. J Human Environ 37, 114-8.

Boyle DG, Hyatt AD, Daszak P, Berger L et al. 2003 - Cryo-archiving of Batrachochytrium dendrobatidis and other chytridiomycetes. Diseases of Aquatic Organisms 56, 59-64.

Braddock A. 2000 - Photography through the microscope: A guide for absolute beginners. Field Mycol 1, 7-8.

Braun U, Crous PW, Groenewald JZ, Scheuer C. 2011 - Pseudovirgaria, a fungicolous hyphomycete genus. IMA Fungus 2, 65-69.

Brook PJ. 1959 - A volumetric spore trap for sampling pastures. New Zealand Journal of Agricultural Research 2, 690-693.

Bruns JA, Zehr JP, Montoya JP, Kustka AB, Capone DG. 2006 - Effect of EDTA additions on natural Trichodesmium spp. (Cyanophyta) populations. J Phycol 42, 900-904. 
Bruns TD. 2012 - The North American Mycoflora project - the first steps on a long journey. New Phytol 196, 972-974.

Bueno L, Gallardo R. 1998 - Preservación de hongos filamentosos en agua destilada estéril. Rev Iberoam Micol 15, 166-168.

Bunse T, Steigleder GK. 1991 - The preservation of fungal cultures by lyophilization. Mycoses 34, $173-176$.

Cai L, Hyde KD, Taylor PWJ, Weir BS et al. 2009 - A polyphasic approach for studying Colletotrichum. Fungal Divers 39, 183-204.

Cai L, Ji KF, Hyde KD. 2006 - Variation between freshwater and terrestrial fungal communities on decaying bamboo culms. Antonie Van Leeuwenhoek 89, 293-301.

Calkins S, Elledge NC, Hanafy RA, Elshahed MS, Youssef N. 2016 - A fast and reliable procedure for spore collection from anaerobic fungi: Application for RNA uptake and long-term storage of isolates. J Microbial Methods 127, 206-213.

Carmichael JW. 1962 - Viability of mold culture stored. Mycologia 54, 432-436.

Carter M, Shieh J. 2015 - Guide to Research Techniques in Neuroscience. Academic Press, London.

Chandler D, Bailey AS, Tatchell GM, Davidson G et al. 2011 - The development, regulation and use of bio-pesticides for integrated pest management. Philos Trans R Soc B: Biol Sci 366, 1987-98

Chesters CGC. 1940 - The Arundel Foray. Trans Br Mycol Soc 24, 1-3.

Chesters CGC. 1948 - A contribution to the study of fungi in the soil. Trans Br Mycol Soc 30, $100-117$.

Chethana KWT, Jayawardena RS, Hyde KD. 2020 - Hurdles in fungal taxonomy: Effectiveness of recent methods in discriminating taxa. Megataxa 001, 114-122.

Chi F, Shen SH, Cheng HP, Jing YX et al. 2005 - Ascending migration of endophytic rhizobia from roots to leaves, inside rice plants and assessment of benefits to rice growth physiology. Appl Environ Microbiol 71, 7271-7278.

Choi YW, Hyde KD, Ho WH. 1999 - Single spore isolation of fungi. Fungal Divers 3, 29-38.

Chomnunti P, Hongsanan S, Aguirre-Hudson B, Tian Q et al. 2014 - The sooty moulds. Fungal Divers 66, 1-36.

Chomnunti P, Schoch CL, Aguirre-Hudson B, Ko-Ko TW et al. 2011 - Capnodiaceae. Fungal Divers 51, 103-134.

Chuang SC, Ho HM, Reynolds N, Smith ME et al. 2017 - Preliminary phylogeny of Coemansia (Kickxellales), with descriptions of four new species from Taiwan. Mycologia 109, 815-831.

Clark TB, Kellen WR, Lindegren JE. 1963 - Axenic culture of two Trichomycetes from California mosquitoes. Nature London 197, 208-209.

Collins MM, Nair SB, Der-Haroutian V, Close D et al. 2005 - Effect of using multiple culture media for the diagnosis of noninvasive fungal sinusitis. Am J Rhinol 19, 41-45.

Connell SL, Padgett DE. 1988 - An improved technique for making permanent slide cultures of fungi. Mycopathologia 101, 165-166.

Coombs JT, Franco CMM. 2003 - Isolation and identification of actinobacteria isolated from surface-sterilized wheat roots. Appl Environ Microbiol 69, 5603-5608.

Crous PW, Slippers B, Wingfield M, Rheeder J et al. 2006 - Phylogenetic lineages in the Botryosphaeriaceae. Stud Mycol 55, 235-253.

Crous PW, Schoch CL, Hyde KD, Wood AR et al. 2009a - Phylogenetic lineages in the Capnodiales. Stud Mycol 64, 17-47.

Crous PW, Verkley GJM, Groenewald JZ, Samson RA. 2009b - Fungal Biodiversity: CBS Laboratory Manual Series, CBS Laboratory Manual Series Volume 1, Westerdijk Fungal Biodiversity Institute, Utrecht.

Daghighi E, Burkhardt U, Filser J, Koehler H. 2016 - Techniques for clearing and mounting Collembola from old ethanol collections. Soil Organisms 88, 89-97. 
Dahlberg KR, Etten J. 1982 - Physiology and biochemistry of fungal sporulation. Annu Rev Phytopathol 20, 281-301.

Dai DQ, Tang LZ, Wang HB. 2018 -A review of bambusicolous ascomycetes. In: Abdul Khalil H (Ed.) Bamboo: Current and Future Prospects, 165 - 183. [ISBN978-1-78923-231-8]. Doi: 10.5772/intechopen.76463.

Dastogeer KMG, Li H, Sivasithamparam K, Jones M, Wylie S. 2018 - Fungal endophytes and a virus confer drought tolerance to Nicotiana benthamiana plants through modulating osmolytes, antioxidant enzymes and expression of host drought responsive genes. Env Exper Bot $149,95-108$.

Davies DR, Theodorou MK, Lawrence MI, Trinci AP. 1993 - Distribution of anaerobic fungi in the digestive tract of cattle and their survival in faeces. J Gen Microbiol 139, 1395-1400.

Davis WH. 1930 - Single Spore Isolation. Proc Iowa Acad Sci 37, 151-159.

Dayarathne M, Boonmee S, Braun U, Crous PW et al. 2016 - Taxonomic utility of old names in current fungal classification and nomenclature: Conflicts, confusion \& clarifications. Mycosphere 7, 1622-1648.

De Gasper AL, Vibrans AC, Funez L, Rigon-Jr M, Bittencourt F, Vieira C. 2014 - Dr. Roberto Miguel Klein Herbarium (FURB), Blumenau, Southern Brazil PhytoKeys 37, 21-37.

De Lima CLF, Lima DX, De Souza CAF, De Oliveira RJV et al. 2018 - Description of mucorpernambucoensis (mucorales, mucoromycota), a new species isolated from the Brazilian upland rainforest. Phytotaxa 350, 274.

De Mattos-Shipley KMJ, Ford KL, Alberti F, Banks AM et al. 2016 - The good, the bad and the tasty: The many roles of mushrooms. Stud Mycol 85, 125-157.

De Souza C, Voigt K, Gurgel L, Cordeiro T et al. 2018 - A new species of Mucor (Mucoromycotina, Mucorales) isolated from an enclave of Upland Atlantic Forest in the semi-arid region of Brazil. Phytotaxa 351(1), 53-62.

De Souza FA, Declerck S. 2003 - Mycelium development and architecture, and spore production of Scutellospora reticulata in monoxenic culture with Ri T-DNA transformed carrot roots. Mycologia 95, 1004-1012.

De Souza LS, Godwin JC, Renshaw MA, Larson E. 2016 - Environmental DNA (eDNA) detection probability is influenced by seasonal activity of organisms. PLoS ONE 11(10), e0165273.

Delly JG. 1988 - Photography through the microscope. Photographic products group, The Eastman Kodak Company, Rochester, New York.

Dentinger BT, Gaya E, O'Brien H, Suz LM et al. 2016 - Tales from the crypt: genome mining from fungarium specimens improves resolution of the mushroom tree of life. Biol J Linn Soc $117,11-32$.

Dhingra OD, Sinclair JB. 1985 - Basic plant pathology methods. CRC Press.

Diehl WW. 1929 - An improved method for sealing microscopic mounts. Science 69, 276-277.

Diogo HC, Sarpieri A, Pires MC. 2005 - Fungi preservation in distilled water. An Bras Dermatol 80, 591-4.

Doilom M, Manawasinghe IS, Jeewon R, Jayawardena RS et al. 2017 - Can ITS sequence data identify fungal endophytes from cultures? A case study from Rhizophora apiculata. Mycosphere 8, 1869-1892.

Dong Z, Canny MJ, McCully ME, Roboredo MR et al. 1994 - A nitrogen fixing endophyte of sugarcane stems. Plant Physiol 105, 1139-1147.

Dubois A. 2010 - Describing new species. Taprobanica 2, 6-24.

Dudley N. 2008 - Guidelines for Applying Protected Area Management Categories. Gland, Switzerland, IUCN

Dudley N, Shadie P, Stolton S. 2013 - Guidelines for applying protected area management categories including IUCN WCPA best practice guidance on Recognising Protected Areas and Assigning Management Categories and Governance Types. Best Practice Protected Area Guidelines Series, 21. 
Duong LM, McKenzie EHC, Lumyong S, Hyde KD. 2008 - Fungal succession on senescent leaves of Castanopsis diversifolia in Doi Suthep-Pui National Park, Thailand. Fungal Divers 30, 23 36.

Ekanayaka AH, Ariyawansa HA, Hyde KD, Jones EB et al. 2017 - Discomycetes: the apothecial representatives of the phylum Ascomycota. Fungal Divers 87, 237-298.

Ellis MB. 1971 - Dematiaceus Hyphomycetes. Commonwealth Mycological Institute, Kew, Surrey, UK.

Elson MK, Schisler DA, Jackson MA. 1998 - Carbon-to-nitrogen ratio, carbon concentration, and amino acid composition of growth media influence conidiation of Helminthosporium solani. Mycologia 90(3), 406-413.

Emerson R. 1958 - Mycological organization. Mycologia 50, 589-621.

Engelkes C, Nuclo R, Fravel D. 1997 - Effect of carbon, nitrogen, and C: N ratio on growth, sporulation and biocontrol efficacy of Talaromyces flavus. Phytopathology 87(5), 500-505.

Evidente A, Cimmino A, Andolfi A, Vurro M et al. 2008 - Phyllostoxin and phyllostin, bioactive metabolites produced by Phyllosticta cirsii, a potential mycoherbicide for Cirsium arvense biocontrol. J Agric Food Chem 56(3), 884-888.

Fairclough A, Loxley P, Johnson D, Mills J. 1985 - An improved fungal mounting technique for Nomarski microscopy. J Biol Educ 19(3), 182-183.

Fei LW, Lu WB, Xu XZ, Yan FC et al. 2019 - A rapid approach for isolating a single fungal spore from rice blast diseased leaves. J Integr Agric 18, 1415-1418.

Fernandes EG, Pereira OL, da Silva CC, Bento CB, de Queiroz MV. 2015 - Diversity of endophytic fungi in Glycine max. Microbiol Res 181, 84-92.

Fisher PJ, Petrini O, Webster J. 1991 - Aquatic hyphomycetes and other fungi in living terrestrial roots of Alnus glutinosa. Mycol Res 95, 543-547.

Fisher PJ, Webster J. 1981 - Ecological studies on aero-aquatic hyphomycetes. In: Wicklow DT, Carroll GC (eds) The fungal community. Basel, Dekker, New York, pp 709-730.

Fiss M, Kucheryava N, Schönherr J, Kollar A et al. 2000 - Isolation and characterization of epiphytic fungi from the phyllosphere of apple as potential biocontrol agents against apple scab (Venturis inaequalis). J Plant Dis Prot 107(1), 1-11.

Foster MS, Mueller GM, Bills GF. 2011 - Biodiversity of Fungi: inventory and monitoring methods. Elsevier.

Frey-Klett P, Chavatte M, Clausse ML, Courrier S et al. 2005 - Ectomycorrhizal symbiosis affects functional diversity of rhizosphere fluorescent pseudomonads. New Phytol 165, 317-328.

Fries EM. 1873 - Observations on Gonnermann and Rabenhorst's Mycologia Europaea. Grevillea $2(14), 1-27$.

Fuhrer BA. 2008 - A Field Companion to Australian Fungi ( $3^{\text {rd }}$ ed.) Bloomings Books, Melbourne.

Gadoury DM, MacHardy WE. 1982 - Preparation and interpretation of squash mounts of pseudothecia of Vennturia inaequalis. Phytopathology 72, 92-95.

Gams W. 1992 - The analyses of communities of saprophytic microfungi with special reference to soil fungi. In: Winterhoff W (Ed) Fungi in vegetation science. Kluwer, Dordrecht.

Gams W. 2015 - An ex-type culture cannot always tell the ultimate truth. IMA Fungus 6(2), 69.

Gan H, Churchill A, Wickings K. 2017 - Invisible but consequential: root endophytic fungi have variable effects on belowground plant-insect interactions. Ecosphere 8, e01710.

Gao L, Sun MH, Liu XZ, Che YS. 2007 - Effects of carbon concentration and carbon to nitrogen ratio on the growth and sporulation of several biocontrol fungi. Mycol Res 111(1), 87-92.

Gardner GA, Szabo I. 1982 - British Columbia pelagic marine Copepoda: an identification manual and annotated bibliography. Can Special Pub Fish Aqua Sci 62, 1-536.

Gargas A, De Priest PT, Grube M, Tehler A. 1995 - Multiple origins of lichen symbioses in fungi suggested by SSU rDNA phylogeny. Science 268, 1492-1495.

Garvetto A, Badis Y, Perrineau M, Rad-Menéndez C et al. 2019 - Chytrid infecting the bloomforming marine diatom Skeletonema sp.: Morphology, phylogeny and distribution of a novel species within the Rhizophydiales. Fungal Biol 123, 471-480. 
Geisen S, Snoek LB, ten Hooven FC, Duyts H et al. 2018 - Integrating quantitative morphological and qualitative molecular methods to analyse soil nematode community responses to plant range expansion. Methods Ecol Evol 9, 1366-1378.

Girometta CE, Bernicchia A, Baiguera RM, Bracco F et al. 2020 - An Italian Research Culture Collection of Wood Decay Fungi. Diversity 12(2), 58.

Glienke C, Pereira OL, Stringari D, Fabris J et al. 2011 - Endophytic and pathogenic Phyllosticta species, with reference to those associated with Citrus black spot. Persoonia 26, 47-56.

Glime JM, Wagner DM. 2017 - Laboratory Techniques: Preservation and Permanent Mounts, Chapt. 2-4. In: Glime JM, Bryophyte Ecology, vol 3, Methods. Ebook sponsored by Michigan Technological University and the International Association of Bryologists.

Goh TK. 1999 - Single-spore isolation using a hand-made glass needle. Fungal Divers 2, 47-63.

Golinska P, Wypij M, Agarkar G, Rathod D et al. 2015 - Endophytic actinobacteria of medicinal plants: diversity and bioactivity. Antonie van Leeuwenhoek 108, 267-289.

Gouda S, Das Sen S, Shin H, Patra JK. 2016 - Endophytes: A Treasure House of Bioactive Compounds of Medicinal Importance. Front Microbiol 7, 1538.

Graham JH. 2001 - What do root pathogens see in mycorrhizas? New Phytol 149, 357-359.

Gregory PH. 1954 - The construction and use of a portable volumetric spore trap Trans Br Mycol Soc 37, 390-404.

Guarro J, Gené J, Stchigel AM. 1999 - Developments in Fungal Taxonomy. Clin Microbiol Rev $12,454-500$.

Guo LD, Huang GR, Wang Y, He WH et al. 2003 - Molecular identification of white morphotype strains of endophytic fungi from Pinus tabulaeformis. Mycol Res 107(6), 680-688.

Guo LY, Michaelides TJ. 1998 - Factors affecting the rate and mode of germination of Mucor piriformis zygospores. Mycol Res 102, 815-819.

Gupta VK, Pandey BK. 2013 - Histopathological Technique for Detection of Fungal Infections in Plants. In: Gupta V, Tuohy M, Ayyachamy M, Turner K, O’Donovan A (eds) Laboratory Protocols in Fungal Biology. Fungal Biology, Springer, New York, NY.

Gupta VK, Tuohy MG, Ayyachamy M, Turner KM, O’Donovan A. 2013 - Laboratory protocols in fungal biology: current methods in fungal biology. Springer Science and Business Media.

Halling RE, Mueller GM. 2005 - Common mushrooms of the Talamanca Mountains, Costa Rica. New York Botanical Garden.

Hamayun M, Seema N, Hussain A, Shah M et al. 2018 - Endophytic fungi promote growth of Zea mays L. under PEG induced drought stress. J Appl Environ Biol Sci 8, 95-101.

Hanafy RA, Elshahed MS, Youssef NH. 2018 - Feramyces austinii, gen. nov., sp. nov., an anaerobic gut fungus from rumen and fecal samples of wild Barbary sheep and fallow deer. Mycologia 110, 513-525.

Hardoim PR, Van Overbeek LS, Berg G, Pirttilä AM et al. 2015 - The Hidden World within Plants: Ecological and Evolutionary Considerations for Defining Functioning of Microbial Endophytes. Microbiol Mol Biol Rev 79, 293-320.

Harley JL, Waid JS. 1955 - A method of studying active mycelia on living roots and other surfaces in the soil. Trans Br Mycol Soc 38, 104-118.

Harris JL. 2000 - Safe, low-distortion tape touch method for fungal slide mounts. J Clin Microbiol 38(12), 4683-4684.

Hartmann A, Chatzinotas A, Assmus B, Kirchhof G. 2000 - Molecular microbial ecology studies on diazotrophic bacteria associated with non-legumes with special reference to endophytic diazotrops. In: Subba Rao NS, Dommergues YR (eds) Microbial interactions in agriculture and forestry, vol 11, Science, Enfield, 1-14.

Hassan SE, Fouda A, Radwan AA, Salem SS et al. 2019 - Endophytic actinomycetes Streptomyces spp mediated biosynthesis of copper oxide nanoparticles as a promising tool for biotechnological applications. JBIC 24, 377-393.

Hawksworth DL 1985 - Fungus Culture Collections as a Biotechnological Resource, Biotechnology and Genetic Engineering Reviews 3, 417-453. 
Hawksworth DL 1991 - The fungal dimension of biodiversity: magnitude, significance, and conservation. Mycol Res 95, 641-655.

Hawksworth DL 2010 - Terms used in bionomenclature: The naming of organisms (and plant communities), Global Biodiversity Information Facility.

Hawksworth DL, Lucking R. 2017 - Fungal diversity revisited: 2.2 to 3.8 million species. Microbiol Spectr 5, 79-95.

Hernández-Restrepo M, Genáe J, Castañeda-Ruiz RF, Mena-Portales J et al. 2017 - Phylogeny of saprobic microfungi from Southern Europe. Stud Mycol 86, 53-97.

Hibbett DS, Ohman A, Glotzer D, Nuhn M et al. 2011 - Progress in molecular and morphological taxon discovery in Fungi and options for formal classification of environmental sequences. Fungal Biol Rev 25, 38-47.

Hildebrand EM. 1938 - Techniques for the Isolation of Single Microorganisms. Bot Rev 4, 627664.

Hill DR. 1996 - Thin Agar Film for Enhanced Fungal Growth and Microscopic Viewing in a New Sealable Fungal Culture Case. J Clin Microbiol 34, 2140-2142.

Homei A. 2006 - Medical mycology development and epidemiology in the USA, UK and Japan. Med Mycol 44, 39-54.

Hongsanan S, Maharachchikumbura SS, Hyde KD, Samarakoon MC et al. 2017 - An updated phylogeny of Sordariomycetes based on phylogenetic and molecular clock evidence. Fungal Divers 84(1), 25-41.

Hooke R. 1665 - Micrographia: Or, Some Physiological Descriptions of Minute Bodies Made by Magnifying Glasses with Observations and Inquiries Thereupon. J. Allestry, printer to the Royal Society, United Kingdom.

Hosaka K, Uno K. 2011 - Assessment of the DNA Quality in Mushroom Specimens: Effect of Drying Temperature. Bull Natl Mus Nat Sci Ser B 37, 101-111.

Hosoya T, Hosaka K, Nam KO. 2017 - A check list of non-lichenized fungi occurring on Fagus crenata, a tree endemic to Japan. Mycology 9, 29-34.

Hoysted GA, Kowal J, Jacob A, Rimington WR et al. 2018 - A mycorrhizal revolution. Curr Opin Plant Biol 44, 1-6.

Hughes SJ. 1953 - Conidiophores, conidia, and classification. Can. J. Bot. 31, 577-659.

Hughes SJ. 1976 - Sooty molds. Mycologia 68, 693-820.

Hughes SJ, Seifert KA. 2012 - Taxonomic and nomenclatural notes on sooty mould name based on species mixtures: Hormiscium handelii and Torula lecheriana. Mycoscience 53, 17-24.

Huhndorf SM. 1991 - A Method for Sectioning Ascomycete Herbarium Specimens for Light Microscopy. Mycologia 83, 520-524.

Humber RA. 1997 - Fungi: Preservation of cultures. In: Lacey LA (ed.) Manual of techniques in insect pathology, $1^{\text {st }}$ edn, Academic Press, London, 269-279.

Hungate RE. 1969 - A roll tube method for cultivation of strict anaerobes. In: Norris R, Ribbons DW (eds) Methods in Microbiology, Academic Press, New York, NY, USA, 3B, 117-132.

Hurdeal VG, Gentekaki E, Hyde KD, Jeewon R. 2020 - Where are the basal fungi? Current status on diversity, ecology, evolution, and taxonomy. Biologia. Doi: 10.2478/s11756-020-00642-4

Hyde KD, Alcorn JL. 1993 - Some disease associated microorganisms on plants of Cape York Peninsula and Torres Strait Islands. Australas Plant Pathol 22, 73-83.

Hyde KD, Bahkali AH, Moslem MA. 2010 - Fungi-an unusual source for cosmetics. Fungal Divers 43, 1-9.

Hyde KD, Chaiwan N, Norphanphoun C, Boonmee S et al. 2018 - Mycosphere notes 169-224. Mycosphere 9(2), 271-430.

Hyde KD, Dong Y, Phookamsak R, Jeewon R et al. 2020b - Fungal diversity notes 1151-1276: taxonomic and phylogenetic contributions on genera and species of fungal taxa. Fungal Diversity 100, 5-277.

Hyde KD, Hongsanan S, Jeewon R, Bhat D et al. 2016 - Fungal diversity notes 367-490: taxonomic and phylogenetic contributions to fungal taxa. Fungal Divers 80, 1-270. 
Hyde KD, Jones EBG, Liu JK, Ariyawansa H et al. 2013 - Families of Dothideomycetes. Fungal Divers 63, 1-313.

Hyde KD, Jones EBG. 2002 - Introduction to fungal succession. In: Hyde KD, Jones EBG (eds) Fungal Succession. Fungal Divers 10, 1-4.

Hyde KD, Norphanphoun C, Maharachchikumbura SSN, Bhat DJ et al. 2020a - Refined Families of Sordariomycetes. Mycosphere 11, 305-1059.

Hyde KD, Soytong K. 2008 - The Fungal endophyte dilemma. Fungal Divers 33, 163-173.

Hyde KD, Xu J, Rapior S, Jeewon R et al. 2019 - The amazing potential of fungi: 50 ways we can exploit fungi industrially. Fungal Divers 97, 1-136.

Isenberg HD. 2007 - Clinical Microbiology Procedures Handbook, Vol. I, II \& III. Am Soc Microbiol, Washington, D.C.

Ismail SI, Batzer JC, Harrington TC, Crous PW et al. 2016 - Ancestral state reconstruction infers phytopathogenic origins of sooty blotch and flyspeck fungi on apple. Mycologia 108(2), 292302.

Jan A, Bhat KM, Bhat SJA, Mir MA et al. 2013 - Surface sterilization method for reducing microbial contamination of field grown strawberry explants intended for in vitro culture. Afr J Biotechnology 12, 5749-5753.

Jeewon R, Hyde KD. 2016 - Establishing species boundaries and new taxa among fungi: recommendations to resolve taxonomic ambiguities. Mycosphere 7, 1669-1677.

Jerônimo GH, Jesus AL, Simmons DR, James TY, Pires-Zottarelli CLA. 2019 - Novel taxa in Cladochytriales (Chytridiomycota): Karlingiella (gen. nov.) and Nowakowskiella crenulata (sp. nov.). Mycologia 1-11.

Jiaojiao S, Wattanachai P, Kasem S. 2016 - Isolation and identification of endophytic fungi from 10 species palm trees. IJAT 12, 349-363.

Jobard M, Rasconi S, Sime-Ngando T. 2010 - Diversity and functions of microscopic fungi: a missing component in pelagic food webs. Aquat Sci 72, 255-268.

Joshi A, Lanjekar VB, Dhakephalkar PK, Callaghan TM et al. 2018 - Liebetanzomyces polymorphus gen. et sp. nov., a new anaerobic fungus (Neocallimastigomycota) isolated from the rumen of a goat. MycoKeys 40, 89-110.

Ju Z, Wang J, Pan SL. 2009 - Isolation and preliminary identification of the endophytic fungi which produce Hupzine A from four species in Hupziaceae and determination of Huperzine A by HPLC. J. Med. Sci. 36, 445-449.

Kamal S, Bhargava KS. 1971 - Studies on Soil Fungi from Teak Forests of Gorakhpur IV. Isolation of Fungi by Soil Plate and Soil Dilution Plate Methods. Sydowia 24, 233-240.

Kannan KP, Thilakavathi R, Madhan KD. 2017 - Biodiversity of endophytic fungi from Mukia maderespatana (L) M. Roem - a first report. J Bacteriol Mycol Open Access 4, 109-113.

Kariman K, Barker SJ, Tibbett M. 2018 - Structural plasticity in root-fungal symbioses: diverse interactions lead to improved plant fitness. Peer J 6, e6030.

Karling JS. 1977 - Chytridiomycetarum Iconographia. Lubrecht-Cramer, Monticello, NY, 414.

Karp G. 2009 - Cell and molecular biology: concepts and experiments. John Wiley and Sons.

Karpov SA, Tcvetkova VS, Mamkaeva MA, Torruella G et al. 2016 - Morphological and Genetic Diversity of Opisthosporidia: New Aphelid Paraphelidium tribonemae gen. et sp. nov. J Eukaryot Microbiol 64, 204-212

Karpov SA, Torruella G, Moreira D, Mamkaeva MA, López-García P. 2017 - Molecular Phylogeny of Paraphelidium letcheri sp. nov. (Aphelida, Opisthosporidia). J Eukaryot Microbiol 64, 573-578.

Kashket ER, Cao ZY. 1995 - Clostridial strain degeneration. FEMS Microbiol Rev 17, 307-315.

Khuna S, Suwannarach N, Kumla J, Meerak J et al. 2019 - Apophysomyces thailandensis (Mucorales, Mucoromycota), a new species isolated from soil in northern Thailand and its solubilization of non-soluble minerals. MycoKeys 45, 75-92.

Kim M, Ahn C, Kim C. 2017 - The Fungicolous Ascomycetes Genus Hypomyces in Korea. Mycobiology 45, 209-212. 
Kinsey GC, Paterson RR, Kelly J. 1999 - Methods for the determination of filamentous fungi in treated and untreated waters. J Appl Microbiol Symp Supp 85, 214-224.

Kirk PM, Cannon PF, Minter DW, Stalpers JA. 2008 - Dictionary of the Fungi. 10 ${ }^{\text {th }}$ Edition, Wallingford, CABI, 22.

Kirsop BE, Doyle A. 1991 - Maintenance of microorganisms and cultured cells, a manual of laboratory methods. $2^{\text {nd }}$ edn. Academic Press, London.

Köhler JR, Casadevall A, Perfect J. 2015 - The Spectrum of Fungi That Infects Humans. Cold Spring Harb Perspect Med. 5, a019273.

Kohlmeyer J, Kohlmeyer E. 1972 - Permanent microscopic mounts. Mycologia 64(3), 666-669.

Kohlmeyer J, Kohlmeyer L. 1979 - Marine mycology: the higher fungi. New York Academic Press.

Krug JC. 2004 - Moist chambers for the development of fungi. In: Mueller GM, Bills GF, Foster MS (eds) Biodiversity of fungi. Inventory and monitoring methods. Elsevier, 589-593.

Kudriavtsev VI, Fateeva MV, Nikitina TN. 1972 - Storage of Collection Yeast Cultures under Mineral Oil. Mikrobiologiia 41, 903-908.

Kurihara Y, Degawa Y, Tokumasu S. 2004 - Two novel kickxellalean fungi, Mycoëmilia scoparia gen. sp. nov. and Ramicandelaber brevisporus sp. nov. Mycol Res 108, 1143-1152.

Kurihara Y, Sukarno N, Ilyas M, Yuniarti E et al. 2008 - Indonesian Kickxellales: two species of Coemansia and Linderina. Mycoscience 49, 250-257.

Lacey J, Dutkiewicz J. 1994 - Bioaerosols and occupational lung diseases. J Aerosol Sci 25, 13711404.

Laird SA. 2002 - Biodiversity and Traditional Knowledge: Equitable Partnerships in Practice. Earthscan publications Ltd, London.

Largent DL. 1986 - How to Identify Mushrooms to Genus I: Macroscopic Features, Mad River Press, 166.

Lawrey JD, Diederich P. 2003 - Lichenicolous Fungi: Interactions, Evolution, and Biodiversity. The Bryologist 106, 80-120.

Leach CM. 1962 - Sporulation of diverse species of fungi under near-ultraviolet radiation. Can J Bot. 40(1), 151-161.

Leonard PL. 2010 - A Guide to Collecting and Preserving Fungal Specimens for the Queensland Herbarium. Queensland Herbarium, Department of Environment and Resource Management, Brisbane.

Letcher PM, Longcore JE, Powell MJ. 2014 - Irineochytrium, a new genus in Chytridiales having zoospores and aplanospores. Mycologia 106(6), 1188-1198.

Leyronas C, Duffaud M, Nicot PC. 2012 - Compared efficiency of the isolation methods for Botrytis cinerea. Mycology 3, 221-225.

Li GJ, Hyde KD, Zhao RL, Hongsanan S et al. 2016 - Fungal diversity notes 253-366: taxonomic and phylogenetic contributions to fungal taxa. Fungal Diversity 78, 1-237.

Li W, Zhou J, Guo SY, Guo LD. 2007 - Endophytic fungi associated with lichens in Baihua mountain of Beijing, China. Fungal Divers 25, 69-80.

Li WJ, Mckenzie EH, Liu JK, Bhat JD et al. 2020 - Taxonomy and phylogeny of hyaline-spored coelomycetes. Fungal Divers 100, 279-801.

Little GN, Gordon MA. 1967 - Survival of fungus cultures maintained under mineral oil for twelve years. Mycologia 59, 733-736.

Liu F, Hyde KD, Cai L. 2011 - Neotypification of Colletotrichum coccodes, the causal agent of potato black dot disease and tomato anthracnose. Mycology. 2, 248-254.

Liu L, Wang CL, Peng WY, Yang J et al. 2015 - Direct DNA extraction method of an obligate parasitic fungus from infected plant tissue. Genet Mol Res 14, 18546-18551.

Lodge DJ, Padamsee M, Matheny PB, Aime MC et al. 2014 - Molecular phylogeny, morphology, pigment chemistry and ecology in Hygrophoraceae (Agaricales). Fungal Divers 64, 1-99.

Longcore JE, Barr DJS, Désaulniers N. 1995 - Powellomyces, a new genus in the Spizellomycetales. Can J Bot 73, 1385-1390. 
Longcore JE, Pessier AP, Nichols DK. 1999 - Batrachochytrium dendrobatidis gen. et sp. nov., a chytrid pathogenic to amphibians. Mycologia 91, 219-227.

Lowe SE, Theodorou MK, Trinci APJ. 1987 - Isolation of anaerobic fungi from saliva and faeces of sheep. J Gen Microbiol 133, 1829-1834.

Lücking R. 2019 - Stop the Abuse of Time! Strict Temporal Banding is not the future of rankBased classifications in Fungi (Including Lichens) and other organisms. Critical Reviews in Plant Sciences 38, 199-253.

Maharachchikumbura SSN, Guo LD, Liu ZY, Hyde KD. 2016 - Pseudopestalotiopsis ignota and Ps. camelliae spp. nov. associated with grey blight disease of tea in China. Mycol Prog 15, 22.

Maharachchikumbura SSN, Hyde KD, Groenewald JZ, Xu J, Crous PW. 2014 - Pestalotiopsis revisited. Stud Mycol 79, 121-186.

Manawasinghe IS, Dissanayake AJ, Li X, Liu M et al. 2019 - High Genetic Diversity and Species Complexity of Diaporthe Associated With Grapevine Dieback in China. Front Microbiol 10, 1936. Doi: $10.3389 /$ fmicb.2019.01936

Mancini K, Sidoriak J. 2017 - Fundamentals of Forensic Photography: Practical Techniques for Evidence Documentation on Location and in the Laboratory. Taylor \& Francis, New York, $1-244$.

Maria GL, Sridhar KR. 2004 - Fungal colonization of immersed wood in mangroves of the southwest coast of India. Canadian Journal of Botany 82, 1409-1418.

Marsh PB, Taylor EE, Bassler LM. 1959 - A guide to the literature of certain effects of light on fungi. Plant Dis Rep Suppl, 261, 251-312.

Martinez-Ordaz VA, Rincon-Castaneda CB, Esquivel-Lopez G, Lazo-Saenz JG, Meraz MTL, Rodríguez VMV. 2002 - Fungal spores in the environment of the asthmatic patient in a semidesert area of Mexico. Rev Alerg Mex 49, 2-7

Masangkay RF, Paulitz TC, Hallett SG, Watson AK. 2000 - Characterization of sporulation of Alternaria alternata $\mathrm{f}$. sp. sphenocleae. Biocontrol Sci Technol 10, 385-397.

Mat-Jalil M, Ibrahim D. 2018 - Fungal endophytes isolated from the leaves of a medicinal plant, Ocimum sanctum Linn and evaluation of their antimicrobial activities. Afri J Microbiol Res 12, 616-622.

Mattos KA, Pádua VL, Romeiro A, Hallack LF et al. 2008 - Endophytic colonization of rice (Oryza sativa L.) by the diazotrophic bacterium Burkholderia kururiensis and its ability to enhance plant growth. An Acad Bras Ciênc 80, 477-493.

McAlpine D. 1906 - The Rusts of Australia. Government Printer, Melbourne.

McCully ME. 2001 - Niches for bacterial endophytes in crop plants: A plant biologist's view. Aus J Plant Physiol 28, 983-990.

McInroy J, Kloepper J. 1995 - Survey of indigenous bacterial endophytes from cotton and sweet corn. Plant Soil 173, 337-342.

McSweeney CS, Denman SE, Mackie RI. 2005 - Rumen bacteria. In: Makkar HPS, McSweeney CS (eds.) Methods in Gut Microbial Ecology for Ruminants. Dordrecht, Netherlands, Springer, pp 23-37.

Meineke EK, Davies CC, Davies TJ. 2018 - The unrealized potential of herbaria for global change biology. Ecol. Monogr. Doi: 10.1101/218776

Meletiadis J, Meis JFGM, Mouton JW, Verweij PE. 2001 - Analyses of Growth Characteristics of Filamentous Fungi in Different Nutrient Media. J Clin Microbiol 39(2), 478-484.

Melotto M, Underwood W, He SY. 2008 - Role of Stomata in Plant Innate Immunity and Foliar Bacterial Diseases. Annu Rev Phytopathol 46, 101-122.

Metcalf WW, Zhang JK, Wolfe RS. 1998 - An anerobic, intrachamber incubator for growth of Methanosarcina spp. on methanol-containing solid media. Appl Environ Microbiol 64, 768770.

Miller TL, Wolin MJ. 1974 - A serum bottle modification of the Hungate technique for cultivating obligate anaerobes. Appl Microbiol 27(5), 985-987. 
Molin R, Horton TR, Trappe JM, Marcot BG. 2011 - Addressing uncertainty: how to conserve and manage rare or little-known fungi. Fungal Ecol 4, 134-146.

Mozley-Standridge SE, Letcher PM, Longcore JE, Porter D, Simmons DR. 2009 - Cladochytrialesa new order in Chytridiomycota. Mycol Res 113:498-507.

Mucciarelli M, Scannerini S, Bertea CM, Maffei ME. 2002 - An ascomycetous endophyte isolated from Mentha piperita L., biological features and molecular studies. Mycologia 94(1), 28-39.

Mueller GM, Bills GF, Foster MS. 2004a - Biodiversity of fungi: inventory and monitoring methods. Elsevier Academic press, London.

Mueller GM, Schmit JP, Ryvarden SM, O’Dell TE. 2004b - Recommended protocols for sampling macrofungi: Inventory and Monitoring Methods. In: Mueller GM, Bills G, Foster MS (eds) Biodiversity of Fungi: Inventory and Monitoring Methods, Elsevier Academic Press, London, 168-172.

Mueller GM, Schmit JP. 2007 - Fungal biodiversity: What do we know? What can we predict? Biodivers Conserv 16, 1-5.

Mueller GM, Schmit JP, Huhndorf SM, O'Dell TE et al. 2004c - Measuring and monitoring diversity of terrestrial and Lignicolous macrofungi: recommended protocols for sampling macrofungi. In: Mueller GM, Bills GF, Foster MS (eds) Biodiversity of Fungi: Inventory and Monitoring Methods, Elsevier Academic Press, London, 123-168.

Muggia L, Grube M. 2018 - Fungal diversity in lichens: from extremotolerance to interactions with algae. Life 8(2), 15. Doi: 10.3390/life8020015

Mukerji KG, Manoharachary C. 2010 - Taxonomy and Ecology of Indian Fungi. I K International Publishing House, 296.

Müller T, Ruppel S. 2014 - Progress in cultivation-independent phyllosphere microbiology. FEMS Microbiol Ecol 87, 2-17.

Murray PR, Baron EJ, Pfaller MA, Tenover FC, Yolken RH. 1995 - Manual of clinical microbiology, 6th ed, ASM Press, Washington DC, 697-797.

Muthukrishnan S, Sanjayan KP, Jahir HK. 2012 - Species composition, seasonal changes and community ordination of alkalotolerant micro fungal diversity in a natural scrub jungle ecosystem of Tamil Nadu, India. Mycosphere 3, 92-109.

Napoli C, Marcotrigiano V, Montagna MT. 2012 - Air sampling procedures to evaluate microbial contamination: a comparison between active and passive methods in operating theatres. BMC pub health 12, 594.

Nebane CLN, Ekpo JA. 1992 - Effect of culture media, temperature and light on radial growth and pycnidium production of cowpea isolates of Phoma bakeriana. Annals of Applied Biology 121(3), 537-544.

Nguyen TT, Duong T, Lee H. 2016 - Characterization of Two New Records of Mucoralean Species Isolated from Gut of Soldier Fly Larva in Korea. Mycobiology 44, 310-313.

Nguyen TT, Park SW, Pangging M, Lee HB. 2018 - Molecular and Morphological Confirmation of Three Undescribed Species of Mortierella from Korea. Mycobiology 47, 31-39.

Nirenberg HI. 1976 - Untersuchungen über die morphologische und biologische Differenzierung in der Fusarium Sektion Liseola. Mitteilungen aus der Biologischen Bundesanstalt fur Landund Forstwirtschaft 169, 1-117.

Noman E, Al-Gheethi AA, Rahman NK, Talip et al. 2018 - Single spore isolation as a simple and efficient technique to obtain fungal pure culture (IConCEES 2017) IOP Conference Series: Earth and Environmental Science, 140, 012055.

O’Connor S, O'Connor PF, Feng HA, Ashley K. 2014 - Gravimetric analyses of particulate matter using air samplers housing internal filtration capsules. Gefahrst Reinhalt Luft, 74(10), 403410 .

Ogawa Y, Hayashi S, Degawa Y, Yaguchi Y. 2001 - Ramicandelaber, a new genus of the Kickxellales, Zygomycetes. Mycoscience 42, 193-199.

Oh SY, Kim M, Eimes JA, Lim YW. 2018 - Effect of fruiting body bacteria on the growth of Tricholoma matsutake and its related molds. PLoS One 13(2), e0190948. 
Olson KD. 1992 - Modified bottle plate for the cultivation of strict anaerobes. J Microbiol Methods 14, 267-269.

Olsson PA, Bååth E, Jacobson I, Söderstrom B. 1996 - Soil bacteria respond to presence of roots but not to mycelium of arbuscular mycorrhizal fungi. Soil Biol Biochem 28, 463-470.

Onion AHS, Allsop D, Eggins HOW. 1981 - Smith's Introduction to Industrial Mycology, 7th Ed., Edward Arnold Publishers Ltd., London.

Onions AHS. 1971 - Chapter IV Preservation of Fungi. Methods in Microbiology 4. In: C. Booth 1971 - Methods in Microbiology, Volume 4 Academic Press 1, 1-794.

Oses R, Valenzuela S, Freer J, Santuentes E, Rodriguez J. 2008 - Fungal endophytes in xylem of healthy Chilean trees and their possible role in early wood decay. Fungal Divers 33, 77-86.

Papagianni M. 2014 - Characterization of fungal morphology using digital image analyses techniques. J Microb Biochem Technol 6(4), 189-194.

Parfrey LW, Lahr DJG, Knoll AH, Katz LA. 2011 - Estimating the timing of early eukaryotic diversification with multigene molecular clocks. Proc Natl Acad Sci USA 108(33), 1362413629.

Parkinson D, Williams ST. 1960 - A method for isolating fungi from soil microhabitats. Plant and Soil 13, 347-355.

Pasquarella C, Albertini R, Dall'Aglio P, Saccani E et al. 2008 - Air microbial sampling: the state of the art. Ig. Sanita Pubbl 64, 79-120.

Paul P, Munkvold GP. 2005 - Influence of temperature and relative humidity on sporulation of Cercospora zeae-maydis and expansion of gray leaf spot lesions on maize leaves. Plant Dis 89(6), 624-630.

Pelczar MJ, Chan ECS, Krieg NR. 1993 - Microbiology: concepts and applications. New York McGraw Hill.

Peršoh D. 2015 - Plant-associated fungal communities in the light of meta'omics. Fungal Divers $75,1-25$.

Persoon CH. 1794 - Dispositio methodica fungorum: A methodical classification of the fungi. Neues Magazin für die Botanik 1, 81-128.

Philipson MN, Blair ID. 1957 - Bacteria in clover root tissue. C J Microbiol 3(2), 125-129.

Phukhamsakda C, McKenzie EH, Phillips AJL, Jones EBG et al. 2020a - Study of fungi associated with Clematis L. (Ranunculaceae) and integrated approach to delimiting species boundaries. Fungal divers 102, 1-203.

Phukhamsakda C, Wijayawardene NN, Ariyawansa HA, Senanayake IC et al. 2020b - Special issue: The contributions of Erio Camporesi. Fungal Divers 100, 1-3.

Pihet M, Carrere J, Cimon B, Chabasse D et al. 2009 - Occurrence and relevance of filamentous fungi in respiratory secretions of patients with cystic fibrosis - a review. Medical Mycology 47(4), 387-397.

Pimentel MR, Molina G, Dionisio AP, Maróstica MR, Pastore GM. 2011 - Use of endophytes to obtain bioactive compounds and their application in biotransformation process. Biotechnol. Res. Int. 2011, 576286. Doi: 10.4061/2011/576286

Pöggeler S, Nowrousian M, Kück U. 2006 - Fruiting-Body Development in Ascomycetes. In: Fischer K (Ed.). The Mycota I: Growth, Differentation and Sexuality. Springer-Verlag Berlin Heidelberg 325-355.

Powell MJ. 2017 - Blastocladiomycota. In: Archibald J, Simpson A, Slamovits C (Eds) Handbook of the Protists. $2^{\text {nd }}$ ed. Springer International Publishing.1497-1521.

Prance M, Fechner N. 2017 - Collecting and preserving fungi specimens, a manual, $2^{\text {nd }}$ ed, Department of Science, Information Technology and Innovation, Brisbane.

Prasad B, Dutt BL, Nagaich BB. 1973 - Inducing sporulation in Alternaria solani. I. Effect of water treatment. Mycopathologia 49(2-3), 141-146.

Radhakrishnan R, Khan AL, Lee IJ. 2013 - Endophytic fungal pre-treatments of seeds alleviates salinity stress effects in soybean plants. J Microbiol 51, 850-857. 
Raja HA, Miller AN, Pearce CJ, Oberlies NH. 2017 - Fungal identification using molecular tools: a primer for the natural products research community J Nat Prod 80(3), 756-770.

Raja S, Subhashini P, Thangaradjou T. 2016 - Differential methods of localisation of fungal endophytes in the sea grasses. Mycology 7, 112-123.

Ramalashmi K, Prasanna Vengatesh K, Magesh K, Sanjana et al. 2018 - A potential surface sterilization technique and culture media for the isolation of endophytic bacteria from Acalypha indica and its antibacterial activity. J Med Plants Studies 6, 181-184.

Rands RD. 1917 - The production of spores of Alternaria solani in pure culture. Phytopathology 7 , 316-317.

Rangaswami G, Mahadevan A. 2006 - Diseases of crops plants in India, $4^{\text {th }}$ Ed., New Delhi Prentice Hall of India.

Rather RA, Srinivasan V, Anwar M. 2018 - Seasonal deviation effects foliar endophyte assemblage and diversity in Asparagus racemosus and Hemidesmus indicus. BMC ecology 18, 52

Ratna Kumar P, Hemanth G, Niharika PS, Kolli SK. 2015 - Isolation and identification of soil mycoflora in agricultural fields at Tekkali Mandal Srikakulam District. Int J Adv Pharmacol 14 (2), 484-490.

Rayner ADM, Todd NK. 1979 - Population and community structure and dynamics of fungi in decaying wood. Adv Bot Res 7, 334-420.

Reynolds CS. 1998 - The state of freshwater ecology. Freshwater Biology 39(4), 741-753.

Richter-Heitmann T, Eickhorst T, Knauth S, Friedrich MW, Schmidt H. 2016 - Evaluation of strategies to separate root-associated microbial communities: A crucial choice in rhizobiome research. Front Microbiol 7, 773.

Riddell RW. 1950 - Permanent stained mycological preparations obtained by slide culture. Mycologia 42, 265-270.

Robert V, Vu D, Amor ABH, van de Wiele N et al. 2013 - MycoBank gearing up for new horizons. IMA Fungus 4(2), 371-379.

Robertson NF. 1954 - Studies on the mycorrhiza of Pinus sylvestris. I. The pattern of development of mycorrhizal roots and its significance for experimental studies. New Phytol 53, 253-283.

Rödel P, Hutter I, Schneider C. 2016 - BSL 2: Endophytes in commercial micropropagation friend or foe? $6^{\text {th }}$ international symposium breeding research on medicinal and aromatic plants, BREEDMAP (Vol 6).

Roeckel-Drevet P, Coelho V, Tourvieille J, Nicolas P, De Labrouhe DT. 1997 - Lack of genetic variability in French identified races of Plasmopara halstedii, the cause of downy mildew in sunflower Helianthus annuus. Can J of Microbiol 43, 260-263.

Rogers LA. 1914 - The preparation of dried cultures. The Journal of Infectious Diseases 14(1), $100-123$.

Roncal T, Ugalde U. 2003 - Conidiation induction in Penicillium. Res Microbiol 154(8), 539-546.

Rosana Y, Matsuzawa T, Gonoi T, Karuniawati A. 2014 - Modified slide culture method for faster and easier identification of Dermatophytes. Microbiology 8(3), 135-139.

Rozas EE, Albano RM, Lôbo-Hajdu G, Müller WEG et al. 2011 - Isolation and cultivation of fungal strains from in vitro cell cultures of two marine sponges (Porifera: Halichondrida and Haplosclerida) Braz J Microbiol 42(4), 1560-1568.

Saad MMG, Ghareeb RY, Saeed AA. 2019 - The potential of endophytic fungi as bio-control agents against the cotton leafworm, Spodoptera littoralis (Boisd.) (Lepidoptera: Noctuidae). Egypt J Biol Pest Control 29, 1-7.

Şakıyan N, İnceoğlu Ö. 2003 - Atmospheric concentration of Cladosporium Link and Alternaria Nees in Ankara and the effects of meteorological factors. Turk J Bot 27, 77-81.

Samarakoon MC, Thongbai B, Hyde KD, Brönstrup M et al. 2020 - Elucidation of the life cycle of the endophytic genus Muscodor and its transfer to Induratia in Induratiaceae fam. nov., based on a polyphasic taxonomic approach. Fungal Divers 101, 177-201.

Sanders ER. 2012 - Aseptic Laboratory Techniques: Plating Methods. J Vis Exp. 63, e3064. 
Sanders WB. 1997 - Fine structural features of rhizomorphs (sensu lato) produced by four species of lichen fungi. Mycological Research 101, 319-328.

Santamaría J, Bayman P. 2005 - Fungal epiphytes and endophytes of coffee leaves (Coffea arabica) Microb Ecol 50, 1-8.

Santana JKG, Seixas AL, Ribeiro LHG, Cardoso ACS et al. 2018 - Staining fungal structures with artificial dyes used in the industry of juices. Ciência Rural 48(9), e20180071.

Santos IM, Abrunhosa L, Venâncio A, Lima N. 2003 - The effect of culture preservation techniques on patulin and citrinin production by Penicillium expansum Link. Lett Appl Microbiol 35, 272-275.

Sathe AV, Dighe S. 1987 - A simple and economic method for long-term preservation of mushroom culture. Current Science 56, 485.

Sati SC. 2006 - Recent Mycological Researches, I. K. International Pvt Ltd.

Satyanarayana T, Deshmukh SK, Deshpande MV. 2019 - Advancing frontiers in Mycology and Mycotechnology: basic and applied aspects of Fungi, $1^{\text {st }}$ ed., Springer Singapore, 675.

Scaltriti S, Cencetti S, Rovesti S, Marchesi I et al. 2007 - Risk factors for particulate and microbial contamination of air in operating theatres. Journal of Hospital Infection 66, 320-326.

Schmit JP, Lodge DJ. 2005 - Classical methods and modern analyses for studying fungal diversity. In: Dighton J, White JF, Oudemans P (Eds) The Fungal Community: Its organization and role in the ecosystem. CRC Press.

Schuck S, Weinhold A, Luu VT, Baldwin IT. 2014 - Isolating Fungal Pathogens from a Dynamic Disease Outbreak in a Native Plant Population to Establish Plant-Pathogen Bioassays for the Ecological Model Plant Nicotiana attenuata. PLoS ONE 9(7), e102915.

Seifert KA, Rossman AY. 2010 - How to describe a new fungal species. IMA Fungus 1(2), 109116.

Selbmann L, de Hoog S, Mazzaglia A, Friedmann EI, Onofri S. 2005 - Fungi at the edge of life: Cryptoendolithic black fungi from Antarctic desert. Stud in Mycol 51(51), 1-32.

Senanayake IC, Crous PW, Groenewald JZ, Maharachchikumbura SSN et al. 2017b - Families of Diaporthales based on morphological and phylogenetic evidence. Stud in Mycol 86, 217-296.

Senanayake IC, Jeewon R, Chomnunti P, Wanasingha DN et al. 2018 - Taxonomic circumscription of Diaporthales based on multigene phylogeny and morphology. Fungal Divers 93(1), $241-$ 443.

Senanayake IC, Jeewon R, Hyde KD, Bhat JD, Cheewangkoon R. 2020 - Taxonomy and phylogeny of Leptosillia cordylinea sp. nov. from China. Phytotaxa 435(3), 213-226.

Senanayake IC, Maharachchikumbura SSN, Hyde KD, Bhat JD et al. 2015 - Towards unraveling relationships in Xylariomycetidae (Sordariomycetes). Fungal Divers 73, 73-144.

Senanayake IC, Maharachchikumbura SSN, Jeewon R, Promputtha I et al. 2017a Morphophylogenetic study of Sydowiellaceae reveals several new genera. Mycosphere 8(1), 172-217.

Senanayake IC, Maharachchikumbura SSN, Mortimer PE, Bhat JD, Hyde KD. 2014 - Vialaeaceae; introducing a novel species Vialaea mangiferae. Sydowia 66, 203-216.

Shah S, Shrestha R, Maharjan S, Selosse M, Pant B. 2018 - Isolation and characterization of plant growth-promoting endophytic fungi from the roots of dendrobium moniliforme. Plants 8(1), 5.

Shahin EA, Shepard JF. 1979 - An efficient technique for inducing profuse sporulation of Alternaria species. Phytopathology 69, 618-620.

Sharma SK, Kumar R, Vaishnav A, Sharma PK et al. 2017 - Microbial cultures: maintenance, preservation and registration. In: Varma A, Sharma AK (Eds) Modern tools and techniques to understand microbes. Springer.

Shepherd CJ, Totterdell CJ. 1988 - Mushrooms and toadstools of Australia. Inkata Press, Melbourne.

Shivas RG, Beasley DR, McTaggart AR. 2014 - Online identification guides for Australian smut fungi (Ustilaginomycotina) and rust fungi (Pucciniales) IMA Fungus 5(2), 195-202. 
Shukla S, Wahla V. 2019 - Influence of different sterilizing methods on isolation endophytic bacteria from Rauvolfia serpentine. The Pharma Innovation Journal 8(1), 38-41.

Sieber T. 2002 - Fungal Root Endophytes. In: The Hidden Half Edition: $4^{\text {th }}$ edn. CRC Press, Taylor \& Francis Group, Boca Raton, USA.

Silva J, Freixo R, Gibbs P, Teixeira P. 2011 - Spray-drying for the production of dried cultures. Int J Dairy Technol 64(3), 321-335.

Silverman DA. 1987 - Microscapes: The hidden art of high technology. Functional Photography 22, 24-29.

Simmonds PM. 1930 - A washing device for isolation work with plant material. Phytopathology 20, 911-913.

Simpson TJ, Lunnon MW, MacMillan J. 1979 - Fungal products. Part 21. Biosynthesis of the fungal metabolite, wortmannin, from 1, 2-13 C2 - acetate. J Chem Soc, Perkin Trans 1, 931-934.

Singh R, Dubey AK. 2015 - Endophytic actinomycetes as emerging source for therapeutic compounds. Indo Global J. Pharm. Sci. 5, 106-116.

Smith AH, Smith HV, Weber NS. 1981 - How to know the non-gilled mushrooms. Q Rev Biol 57(2), 192.

Smith G. 1969 - An Introduction to Industrial Mycology, $6^{\text {th }}$ ed., Arnold Ltd, London, UK.

Smith JE, Berry DR. 1974 - An introduction to the biochemistry of fungal development. Academic Press, London.

Smith O, Onions AHS. 1994 - Preservation and maintaince of living fungi. CAB International, UK

Smith P. 2019 - Formula for chemical stains and reagents. The British Mycological Society. https://www.britmycolsoc.org.uk/mycology/microscopy/reagents (Accessed on 1 July 2020).

Smith RL, Sawbridge T, Mann R, Kaur J et al. 2020 - Rediscovering an old foe: Optimised molecular methods for DNA extraction and sequencing applications for fungarium specimens of powdery mildew (Erysiphales). PLoS ONE 15(5), e0232535.

Stadler M, Læssøe T, Fournier J, Decock C et al. 2014 - A polyphasic taxonomy of Daldinia (Xylariaceae). Stud Mycol 77, 1-143.

Steiman R, Guiraud P, Sage L, Seigle-Murandi F, Lafond JL. 1995 - Mycoflora of soil around the Dead Sea I - Ascomycetes (including Aspergillus and Penicillium), Basidiomycetes, Zygomycetes. System Appl Microbiol 18, 310-317.

Stone JK, Polishook JD, White JF. 2004 - Endophytic Fungi. In: GM. Mueller, G. Bills, \& M. S. Foster (Eds), Biodiversity of Fungi: Inventory and Monitoring Methods, 241-270. San Diego, CA: Elsevier Academic Press.

Straatsma G, Ayer F, Egli S. 2001 - Species richness, abundance, and phenology of fungal fruiting bodies over 21 years in a Swiss forest plot. Mycol Res 105, 515-523.

Strauss T, Bothab A, Greyling D, Mostert T et al. 2000 - Development and testing of selective media for mucoralean fungi. South African Journal of Science 96, 597.

Strobel G, Yang X, Sears J, Kramer R et al. 1996 - Taxol from Pestalotiopsis microspora, an endophytic fungus of Taxus wallachiana. Microbiology 142(2), 435-440.

Su YY, Noireung P, Liu F, Hyde KD et al. 2011 - Epitypification of Colletotrichum musae, the causative agent of banana anthracnose. Mycoscience 52, 376-382.

Su YY, Qi YL, Cai L. 2012 - Induction of sporulation in plant pathogenic fungi. Mycology 3, 195200.

Subramanian CV. 1983 - Hyphomycetes taxonomy and biology. London Academic Press.

Sugita N, Ebihara A, Hosoya T, Jinbo U et al. 2020 - Non-destructive DNA extraction from herbarium specimens: a method particularly suitable for plants with small and fragile leaves. J Plant Res 133, 133-141.

Sullivan DJ, Moran GP. 2015 - Review of human pathogenic fungi: molecular biology and pathogenic mechanisms. Front Microbiol 6, 82.

Sun JZ, Liu XZ, McKenzie EH, Jeewon R et al. 2019 - Fungicolous fungi: terminology, diversity, distribution, evolution, and species checklist. Fungal Divers 95, 337-430. 
Sun MH, Gao L, Liu XZ, Wang JL. 2009 - Fungal sporulation in two-stage cultivation. Mycosystema 28(1), 64-72.

Sun X, Guo LD. 2012 - Endophytic fungal diversity: review of traditional and molecular techniques. Mycology 3(1), 65-76.

Sutton BC. 1980 - The Coelomycetes. Fungi imperfecti with pycnidia, acervuli and stromata. CMI, Kew, 1-696.

Suzuki G, Wang Y, Kubo K, Hirata E et al. 2018 - Global study of holistic morphological effectors in the budding yeast Saccharomyces cerevisiae. BMC Genomics 19, 149.

Swathi-Sri A, Subrahmanyam A. 2017 - Mucorimietiana sp. nov. Curr Trends Biotechnol and Pharm 11(1), 99-102.

Swedlow JR. 2012 - Innovation in biological microscopy: Current status and future directions. Bioessays 34(5), 333-340.

Tansey MR. 1984 - Efficient isolation of thermophilic and thermotolerant mucoralean fungi. Mycopathologia 85, 31-42.

Tatiana TMS, Rodrigues LA, Dhingra OD, Eduardo SGM. 2010 - In vitro production of conidia of Alternaria solani. Trop Plant Pathol 35, 1-8.

Taylor JW. 2011 - One Fungus = One Name: DNA and fungal nomenclature twenty years after PCR. IMA Fungus 2(2), 113-120.

Taylor JW, Ellison CE. 2010 - Mushrooms: Morphological complexity in the fungi. Proceedings of the National Academy of Sciences 107(26), 11655-11656.

Thiers BM. 2020 - Index Herbariorum: A global directory of public herbaria and associated staff. New York Botanical Garden's Virtual Herbarium. New York Botanical Garden, Bronx, New York, USA. http://sweetgum.nybg.org/science/ih/ [accessed 20 January 2020]

Thiers BM, Halling RE. 2018 - The Macrofungi collection consortium. Appl Plant Sci 6(2), e1021.

Thiers BM, Wilson N, Halling RE. 2013 - Making scientific vouchers of North American macrofungi: how documenting the fungi you encounter contribute to the mycoflora of North America. McIlvainea 22, 19-24.

Thorn RG, Reddy CA, Harris D, Paul E. 1996 - Isolation of saprophytic Basidiomycetes from soil. Applied and Environmental Microbiology 62(11), 4288-4292.

Timberlake WE. 1980 - Developmental gene regulation in Aspergillus nidulans. Dev Biol. 78, 497-510.

Timnick MB, Lilly VG, Barnett HL. 1951 - The effect of nutrition on the sporulation of Melanconium Fuligineum in culture. Mycologia 43(6), 625-634.

Toghueo RMK, Ejiya IE, Sahal D, Yazdani SS, Boyom FF. 2017 - Production of cellulolytic enzymes by endophytic fungi isolated from Cameroonian medicinal plants. Int $\mathbf{J}$ Curr Microbiol App Sci 6(2), 1264-1271.

Tokumasu S. 1998 - Fungal succession on pine needles fallen at different seasons: The succession of interior colonizers. Mycoscience 39, 409-416.

Tolulope RA, Adeyemi AI, Erute MA, Abiodun TS. 2015 - Isolation and screening of endophytic fungi from three plants used in traditional medicine in Nigeria for antimicrobial activity. Int $\mathbf{J}$ Green Pharm 9, 58-62.

Tonouchi A. 2009 - Isolation and characterization of a novel facultative anaerobic filamentous fungus from Japanese rice field soil. Int J Microbiol, 1-9.

Torres MS, White JF, Hinton DM, Bacon CW. 2011 - Endophyte-mediated adjustments in host morphology and physiology and effects on host fitness traits in grasses. Fungal Ecology 5(3), 322-330.

Trigiano RN, Ownley BH. 2017 - Identifying obligate, biotrophic fungi (and hosts) using the sequence of the internal transcribed spacer (ITS) region. In: Trigiano RN, Ownley BH 2017 Plant Pathology Concepts and Laboratory Exercises, $3^{\text {rd }}$ Ed., Taylor and Francis, Boca Raton, FL, 483-494. 
Tsudome M, Deguchi S, Tsujii K, Ito S, Horikoshi K. 2009 - Versatile solidified nanofibrous cellulose-containing media for growth of extremophiles. Appl Environ Microbiol 75(13), 4616-4619.

Turland NJ, Wiersema JH, Barrie FR, Greuter W et al. 2018 - International Code of Nomenclature for algae, fungi, and plants (Shenzhen Code) adopted by the Nineteenth International Botanical Congress Shenzhen, China, July 2017. Koeltz Botanical Books.

Udayanga D, Castlebury LA, Rossman AY, Chukeatirote E, Hyde KD. 2014 - Insights into the genus Diaporthe: phylogenetic species delimitation in the D. eres species complex. Fungal Divers 67, 203-229.

Vánky K, Shivas RG. 2008 - Fungi of Australia: The Smut Fungi, CSIRO Publishing, Melbourne.

Vasilyeva LN, Stephenson SL. 2010 - Biogeographical patterns in pyrenomycetous fungi and their taxonomy. Mycotaxon 114, 281-303.

Verkley G, Perrone G, Piña M, Scholz AH et al. 2020 - New ECCO model documents for Material Deposit and Transfer Agreements in compliance with the Nagoya Protocol. FEMS Microbiology Letters 367(5). Doi: 10.1093/femsle/fnaa044

Vignesh R, Swathirajan CR, Solomon S, Shankar EM et al. 2013 - Iodine-glycerol as an alternative to lactophenol cotton blue for identification of fungal elements in clinical laboratory. Indian $\mathbf{J}$ Med Microbiol 31(1), 93-94.

von Arx JA, Müller E. 1975 - A re-evaluation of the bitunicate ascomycetes with keys to families and genera. Stud in Mycol 9, 1-159.

Waksman SA. 1927 - Principles of soil microbiology. Williams \& Wilkins, Baltimore.

Wanderley-Costa IP, Cavalcanti MAQ, Fernandes MJS, Lima DM. 2006 - Hyphomycetes from soil of an area affected by copper mining activities in the State of Bahia, Brazil. Braz $\mathbf{J}$ Microbiol 37, 290-295.

Wang LE, Xiong P, Strom SS, Goldberg LH et al. 2005 - In vitro sensitivity to ultraviolet light and skin cancer risk: a case-control analyses. J Natl Cancer Inst 97, 1822-1831.

Wang WX, Lei X, Ai HL, Bai X et al. 2019 - Cytochalasans from the endophytic fungus Xylaria cf. curta with resistance reversal activity against fluconazole-resistant Candida albicans. Org. Lett $21,1108-1111$.

Warcup JH. 1950 - The soil-plate method for isolation of fungi from soil. Nature 166, 117-118.

Warcup JH. 1955 - Isolation of fungi from hyphae present in soil. Nature 175, 953-954.

Warcup JH. 1960 - Methods for isolation and estimation of activity of fungi in soil. The Ecology of Soil Fungi, Liverpool University Press.

Wardle DA, Lavelle P. 1997 - Linkages between soil biota, plant litter quality and decomposition. In: Cadisch G, Giller KE (Eds) Driven by Nature: Plant Litter Quality and Decomposition. Wallingford (UK): CAB International.

Weber RJ, McMurry PH, Mauldin RL, Tanner DJ et al. 1999 - New Particle Formation in the Remote Troposphere: A Comparison of Observations at Various Sites. Geophysical Research Letters 26(3), 307-310.

Webster J, Descals E. 1981 - Morphology, distribution, and ecology of conidial fungi in freshwater habitats. In: Cole GT, Kendrick B (Eds) Biology of conidial fungi. New York Academic Press.

Wennström A. 1993 - Systemic fungal diseases in natural plant populations. UMEÀ Universitet Doctoral Dissertation. Department of Ecological Botany.

Whitman WB, Coleman DC, Wiebe WJ. 1998 - Perspective: Prokaryotes: the unseen majority. Proc Natl Acad Sci USA 95, 6578-6583.

Wibberg D, Stadler M, Lambert C, Bunk B et al. 2020 - High quality genome sequences of thirteen Hypoxylaceae (Ascomycota) strengthen the phylogenetic family backbone and enable the discovery of new taxa. Fungal Divers. Doi: 10.1007/s13225-020-00447-5

Wijayawardene NN, Hyde KD, Bhat DJ, Camporesi E et al. 2014 - Camarosporium-like species are polyphyletic in Pleosporales; introducing Paracamarosporium and Pseudocamarosporium gen. nov. in Montagnulaceae. Cryptogam Mycol 35(2), 177-198. 
Wijayawardene NN, Hyde KD, Wanasinghe DN, Papizadeh M et al. 2016 - Taxonomy and phylogeny of dematiaceous coelomycetes. Fungal Divers 77, 1-316.

Wijayawardene NN, Hyde KD, Al-Ani LKT, Tedersoo L et al. 2020 - Outline of Fungi and fungus-like taxa. Mycosphere 11(1), 1060-1456.

Williams JG, Kubelik AR, Livak KJ, Rafalski JA, Tingey SV. 1990 - DNA polymorphisms amplified by arbitrary primers are useful as genetic markers. Nucleic acids res 18, 65316535.

Willis CG, Ellwood ER, Primack RB, Davis CC et al. 2017 - Old plants, new tricks: phenological research using herbarium specimens. Trends Ecol Evol 32, 531-546.

Willis KJ. 2018 - State of the World's Fungi 2018. Royal Botanic Gardens, Kew.

Wingfield MJ, de Beer ZW, Slippers B, Wingfield BD et al. 2012 - One fungus, one name promotes progressive plant pathology. 13(6), 604-613.

Wolfe RS. 2011 - Techniques for cultivating methanogens. Methods Enzymol, 494, 1-22.

Wongkanoun S, Wendt L, Stadler M, Luangsa-ard J, Srikitikulchai P. 2019 - A novel species and a new combination of Daldinia from Ban Hua Thung community forest in the northern part of Thailand. Mycol Progress 18, 553-564.

Wright BE. 1979 - Causality in biological systems. Trends Biochem Sci 4, 110-111.

Wu B, Hussain M, Zhang WW, Stadler M et al. 2019 - Current insights into fungal species diversity and perspective on naming the environmental DNA sequences of fungi. An International Journal on Fungal Biology 10(3), 127-140.

Wu Q, Thiers BM, Pfister DH. 2004 - Preparation, preservation, and use of fungal specimens in herbaria. In: Mueller GM, Bills GF, Foster MF (Eds.) Biodiversity of Fungi. Burlington, Academic Press.

Wulandari N, To-Anun C, Hyde K, Duong L et al. 2009 - Phyllosticta citriasiana sp. nov., the cause of Citrus tan spot of Citrus maxima in Asia. Fungal Divers 34, 23-39.

Xia Y, Sahib MR, Amna A, Opiyo SO et al. 2019 - Culturable endophytic fungal communities associated with plants in organic and conventional farming systems and their effects on plant growth. Sci Rep 9, 1-10.

Xu JW, Zhao W, Xu YN, Zhong JJ. 2012 - Isolation and analyses of differentially expressed genes during asexual sporulation in liquid static culture of Ganoderma lucidum by suppression subtractive hybridization. Mol Biol Rep 39(4), 3603-3610.

Xu LL, Li F, Xie HY, Liu XZ. 2009 - A novel method for promoting conidial production by a nematophagous fungus, Pochonia chlamydosporia AS6.8. World J Micro Biotechnol 25(11), 1989-1994.

Yazdany S, Lashkari KB. 1975 - Effect of pH on sporulation of Bacillus stearothermophilus. Appl Microbiol 30(1), 1-3.

Yoshida S, Shirata A. 2000 - Biotin induces sporulation of mulberry anthracnose fungus, Colletotrichum dematium. J Genl Plant Pathol 66, 117-122.

Young AM. 2005 - A Field Guide to the Fungi of Australia. UNSW Press, Sydney.

Yu Y, Hube B, Kämper J, Meyer V, Krappmann S. 2017 - When green and red mycology meet: Impressions from an interdisciplinary forum on virulence mechanisms of phyto- and humanpathogenic fungi. Virulence 8(7), 1435-1444.

Zeng L, Tu XL, Dai H, Han FM et al. 2019 - Whole genomes and transcriptomes reveal adaptation and domestication of pistachio. Genome Biol 20, 79.

Zeng X, Jeewon R, Wen T, Hongsanan S et al. 2018 - Simplified and efficient DNA extraction protocol for Meliolaceae specimens. Mycol Progress 17, 403-415.

Zhang K, Su YY, Cai L. 2013 - An optimized protocol of single spore isolation for fungi. Cryptogam Mycol 34(4), 349-356.

Zhang T, Wei XL, Zhang YQ, Liu HY, Yu LY. 2015 - Diversity and distribution of lichenassociated fungi in the Ny-Ålesund Region (Svalbard, High Arctic) as revealed by 454 pyrosequencing. Sci Rep 5, 14850. 
Zhang W, Wolf TM, Bailey KL, Mortensen K, Boyetchko SM. 2003 - Screening of adjuvants for bioherbicide formulations with Colletotrichum spp. and Phoma spp. Biol Control 26, 95-108.

Zhong R, Xia C, Ju Y, Li N et al. 2017 - Effects of Epichloë gansuensis on root associated fungal communities of Achnatherum inebrians under different growth conditions. Fungal Ecol 31, 29-36.

Zhou D, Hyde KD. 2002 - Fungal succession on bamboo in Hong Kong. Fungal Divers 10, 213227. 